Psychophysical methods

for improved observation of 



\section{PSYCHOPHYSICAL METHODS FOR IMPROVED OBSERVATION OF NOCICEPTIVE PROCESSING}

Robert J. Doll 
The research is supported by the Dutch Technology Foundation STW, which is part of the Netherlands Organization for Scientific Research (NWO), and partly funded by the Ministry of Economic Affairs (project number 10740).

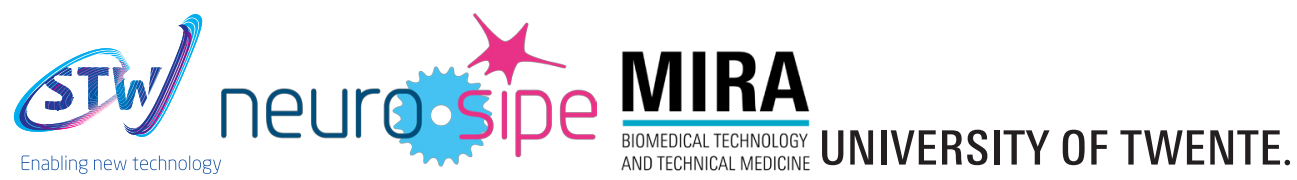

Cover design: Esther Ris

Printing: Gildeprint Drukkerijen, Enschede, the Netherlands

ISBN: 978-90-365-4037-7

DOI: $10.3990 / 1.9789036540377$

\section{(C) Robert J. Doll, 2016}

All rights reserved. No part of this publication may be reproduced or transmitted in any form or by any means, electronic or mechanical, including photocopy, recording or any information storage or retrieval system, without permission in writing from the author. 


\title{
PSYCHOPHYSICAL METHODS FOR IMPROVED OBSERVATION OF NOCICEPTIVE PROCESSING
}

\section{PROEFSCHRIFT}

\author{
ter verkrijging van \\ de graad van doctor aan de Universiteit Twente, \\ op gezag van de rector magnificus, \\ prof. dr. H. Brinksma, \\ volgens besluit van het College voor Promoties \\ in het openbaar te verdedigen op \\ vrijdag 4 maart 2016 om 16:45 uur \\ door \\ Robert Johannes Doll \\ geboren op 26 juli 1988 \\ te 's-Gravenhage
}


Dit proefschrift is goedgekeurd door:

De promotor: prof. dr. ir. P.H. Veltink

De copromotor: dr. ir. J.R. Buitenweg 
Samenstelling promotiecommissie

Voorzitter en secretaris

Prof. dr. P.M.G. Apers Universiteit Twente

Promotor

Prof. dr. ir. P.H. Veltink Universiteit Twente

\section{Copromotor}

Dr. ir. J.R. Buitenweg Universiteit Twente

\section{Leden}

Prof. dr. ir. M.J.A.M. van Putten

Universiteit Twente

Prof. dr. R.J.A. van Wezel

Universiteit Twente

Apl. prof. dr. D. Kleinböhl

Central Institute of Mental Health, Mannheim, Germany

Dr. R.S.G.M. Perez

VU medisch centrum, Amsterdam

Dr. H.P.A. van Dongen

St. Antonius ziekenhuis, Nieuwegein

\section{Paranimfen}

Arjan van der Velde

Joris Oosterhuis 



\section{CONTENTS}

Chapter 1

Introduction

PART I

Psychophysical methods for observation of sensory function

Chapter 2

Tracking of nociceptive thresholds using adaptive psychophysical methods

Chapter 3

Observation of time-dependent psychophysical functions

and accounting for threshold drifts

Chapter 4

Characterization of a psychophysical method for

simultaneous tracking of multiple non-stationary thresholds

\section{PART II}

Observation of nociceptive processing using electrocutaneous stimulation 77

\section{Chapter 5}

Effect of temporal stimulus properties on the nociceptive

detection probability using intra-epidermal electrical stimulation

Chapter 6

Responsiveness of electrical nociceptive detection

thresholds to capsaicin (8\%) induced changes in nociceptive processing

Chapter 7

General discussion

Summary

Samenvatting

Dankwoord

Biography

List of publications 

Chapter 1

Introduction 
Even though pain is usually not considered a pleasant experience, the perception of pain is important and crucial in our daily life. For example, a fall to the ground leads to an unpleasant, likely painful, experience directing the attention towards possible bruises and scrapes. The International Association for the Study of Pain (IASP) (2011) defines pain as an "unpleasant sensory and emotional experience associated with actual or potential tissue damage, or described in terms of such damage." This definition indicates the complex nature of 'pain' as a combination of neurological, physiological, and psychological factors. In fact, the primary function of pain is to warn and protect the body from (further) tissue damage. A lack of pain, for example in patients suffering congenital insensitivity to pain, is considered dangerous as it is difficult or even impossible to identify cuts, infections, or bone fractures (Daneshjou, Jafarieh, \& Raaeskarami, 2012; Nagasako, Oaklander, \& Dworkin, 2003). Therefore, pain perception can be considered an important 'feature' of the human body. The perception of pain varies between individuals, for example differences exist between sex (e.g., Mogil, 2012), age (e.g., Washington, Gibson, \& Helme, 2000), and ethnic background (e.g., Edwards, Doleys, Fillingim, \& Lowery, 2001). Moreover, stress or fear can alter the perception of pain within an individual (e.g., Keefe, Lumley, Anderson, Lynch, \& Carson, 2001).

Due to tissue damage, for example a scrape caused by a fall to the ground, a chain of chemical reactions results in an increase in inflammation and blood flow towards the damage which results in an acute pain response, but also starts the healing process (Millan, 1999; Woolf \& Ma, 2007). With the healing process, the acute pain reduces over time until the site of tissue damage is no longer painful. However, in some cases, the pain outlasts the healing process and no longer serves as a protecting mechanism (Woolf \& Salter, 2000). The pain can become persistent and can have a negative impact on the quality of life (Hunfeld et al., 2001). Persistent pain is considered as chronic when it is present for more than 1 to 6 months (IASP, 1994; Treede et al., 2015). The prevalence rate of chronic pain worldwide is estimated to range between $10 \%$ and $55 \%$ (Harstall \& Ospina, 2003; Tsang et al., 2008). In the Netherlands, the prevalence rate of chronic pain is estimated to be about 18\% (Bekkering et al., 2011).

A common cause of chronic pain is surgery (Crombie, Davies, \& Macrae, 1998). In fact, about $20 \%$ of patients attending outpatient clinics suffer from persisting or chronic pain due to surgical interventions (Crombie et al., 1998). About $80 \%$ of patients undergoing surgery experience acute post-surgical pain, of which 39\% report the pain as severe or extreme (Apfelbaum, Chen, Mehta, \& Gan, 2003). Persisting post-surgical pain (PPSP) is a frequently occurring problem (Kehlet, Jensen, \& Woolf, 
2006; Macrae, 2001, 2008; Perkins \& Kehlet, 2000). Surgical interventions, such as thoracotomy, mastectomy, amputation, or inguinal hernia, are associated with chronic pain development. Estimated prevalence rates depend on the type of surgical intervention and range from $30 \%$ to $50 \%$ in major surgical interventions and range from $10 \%$ to $15 \%$ in minor surgical interventions (Crombie et al., 1998; Kehlet et al., 2006; Macrae, 2001; Perkins \& Kehlet, 2000).

Pain treatments are, once chronic pain has been established, relatively ineffective (Apfelbaum et al., 2003). At best, 25 to $30 \%$ of the patients achieve a $50 \%$ pain reduction (Saarto \& Wiffen, 2007; Wiffen, McQuay, Edwards, \& Moore, 2005). Besides possible risk factors of developing PPSP, such as type of surgical intervention, gender, age, and psychological state (Perkins \& Kehlet, 2000), multiple studies have suggested a link between PPSP and malfunction in the nociceptive system (Kehlet et al., 2006; Macrae, 2001; Wilder-Smith \& Arendt-Nielsen, 2006; Wilder-Smith, Schreyer, Scheffer, \& Arendt-Nielsen, 2010). Additionally, patients who are already diagnosed with nociceptive disease prior to surgery might be more susceptible to PPSP (D'Apuzzo, Cabanela, Trousdale, \& Sierra, 2012). Observation of the nociceptive system and its underlying mechanisms may improve the understanding of the contributions of nociceptive mechanisms in PPSP development. Prior to discussing observation techniques, relevant neurophysiology of pain is briefly discussed in the next section.

\section{NEUROPHYSIOLOGY OF PAIN}

A noxious stimulus activates a chain of reactions in the nociceptive system starting at a peripheral level where the stimulus is translated into neural activity through nociceptive specific receptors and fibers. The peripheral activity is then transmitted and modulated on several locations along ascending pathways through the dorsal horn and spinal cord into the brain. Descending pathways from supraspinal levels to the dorsal horn modulate the excitatory activity of ascending mechanisms. The perceived strength and quality of a stimulus depends on the modulation of the induced neural activity by peripheral mechanisms (Mendell, 2011) and central ascending and descending mechanisms (Bromm \& Lorenz, 1998; Sandkühler, 2009; Woolf, 2011).

In healthy humans, the ascending and descending mechanisms involved in nociceptive stimulus processing are in balance. The sensitizing effects caused by ascending mechanisms are eventually counteracted by the inhibiting effects of descending mechanisms (Wilder-Smith \& Arendt-Nielsen, 2006; Woolf \& Salter, 2000). Failures in the balance between both pathways may result in prolonged modifications of 
nociceptive processing. Malfunctioning nociceptive mechanisms, for example due to increased ascending sensitization or failing inhibiting descending processes may lead to generalized hyperalgesia, which, in turn, may lead to chronic pain disorders. A brief description of ascending and descending nociceptive processing is provided in the following sections.

\section{Peripheral processing}

Unlike peripheral tactile receptors, which have specialized corpuscular endings (e.g., Merkel and Meissner receptors), nociceptive receptors, or nociceptors, are relatively unspecialized free nerve endings (Dubin \& Patapoutian, 2010; Woolf \& Ma, 2007). Peripheral nociceptors reside mostly in the epidermis and are therefore closest to the skin surface in comparison with other cutaneous modalities. Noxious stimuli activate the nociceptors and induce electrical action potentials through either A $\delta$-fibers or C-fibers to their synapses in the dorsal horn. In comparison with the diameter of tactile myelinated A $\beta$-fibers ( $\varnothing$ 6-12 $\mu \mathrm{m}$ ), myelinated A $\delta$-fibers ( $\varnothing$ 1-6 $\mu \mathrm{m}$ ) and unmyelinated C-fibers $(\varnothing 0.2-1.5 \mu \mathrm{m})$ are relatively thin.

Due to their smaller diameter and, in case of C-fibers, lack of a myelin sheath, the conduction velocity of nociceptive A $\delta$-fibers and C-fibers $(5-30 \mathrm{~m} / \mathrm{s}$ and $0.5-2 \mathrm{~m} / \mathrm{s}$, respectively) is slower than the conduction velocity of the thicker tactile $A \beta$-fibers $(35-75 \mathrm{~m} / \mathrm{s})$. The pain excited by activation of A $\delta$-fibers and C-fibers are classified as a sharp first pain and dull second pain, respectively (Purves et al., 2008). Normally, the nociceptors only respond to noxious stimuli, such as pinching, heat, or chemicals. However, during inflammation (e.g., tissue damage), the activation threshold to noxious stimuli is decreased and normally innocuous stimuli may become painful and are referred to as hyperalgesia and allodynia, respectively (Sandkühler, 2009). This partly explains why touching a graze is painful and is referred to as peripheral sensitization (Schaible, 2007).

\section{Central processing}

The afferent fibers synapse with dorsal horn neurons in the grey matter in the dorsal horn. A $\delta$-fibers project mainly to laminae I and V, C-fibers to laminae I and II, and non-nociceptive A $\beta$-fibers to laminae III to V (Todd, 2010). Dorsal horn neurons are either nociceptive specific (NS) neurons, wide dynamic range (WDR) neurons, or interneurons (D’Mello \& Dickenson, 2008; Dubin \& Patapoutian, 2010). A $\delta$-fibers and C-fibers synapse with NS neurons in laminae I and II, and A $\delta$-fibers and A $\beta$-fibers synapse with WDR neurons in lamina V. The interneurons, which can be found in 
and between all laminae, receive input from afferent fibers, as well as from descending supraspinal levels and play an important role in processing tactile and nociceptive neural activity (West, Bannister, Dickenson, \& Bennett, 2015).

Input from the periphery can be processed by several modulatory mechanisms. One of the most important breakthroughs in pain research was the 'Gate control theory', as proposed by Melzack and Wall (1965). Their theory states that pain is modulated in the dorsal horn by input from several types of nerve fibers, including those coming from innocuous stimuli, and that tactile activation 'closes the gate', inhibiting nociceptive input (Moayedi \& Davis, 2013). Other examples demonstrating neural plasticity in the dorsal horn are wind-up effects, short term plasticity, and long term plasticity. The latter two examples have inhibiting or facilitating effects on the postsynaptic potentials after multi-pulse stimulation and effectively decrease or increase the postsynaptic neural activity, respectively. Wind-up effects are induced by low-frequent C-fiber stimulation and is observed as an increase in perceived pain intensity of applied stimuli over time $(\mathrm{Li}$, Simone, \& Larson, 1999). An important factor in persisting and chronic pain disorders is central sensitization which expresses itself as a higher postsynaptic neural activity given the same incoming peripheral neural activity (Latremoliere \& Woolf, 2009).

The NS and WDR neurons project from the dorsal horn to various parts in the thalamus through the lateral and anterior spinothalamic tract, respectively (Mendell, 2011). From the thalamus, neurons are projected deeper into the brain. Cortical structures involved in nociceptive processing are the primary and somatosensory cortices (SI and SII), insular cortex (IC), and the anterior cingulated cortex (ACC). While the SI and SII are involved in discriminative tasks, the IC and ACC are responsible for the quality of perception and emotional and decisive aspects (Wall \& Melzack, 1999).

The neural activity taking place at ascending pathways are is not only modulated by ascending mechanisms, but also by descending mechanisms originating from supraspinal structures. Nociceptive input can be facilitated or inhibited by cortical activation of, for example, the periaqueductal grey and rostroventral medulla. Another example is diffuse noxious inhibitory control (DNIC), which is related to the pain-inhibits-pain phenomenon (Le Bars, Dickenson, \& Besson, 1979a, 1979b; Woolf \& Salter, 2000). DNIC has a heterotopic inhibitory effect on mostly WDR and some NS neurons in the dorsal horn (Le Bars, 2002). 


\section{OBSERVATION OF NOCICEPTIVE PROCESSING}

To understand the mechanisms involved in the processing of painful stimuli, and to study nociceptive mechanisms involved in PPSP, it is important to quantify pain perception. However, due to its complex and subjective nature, the quantification of pain perception is difficult. Unidimensional questionnaires such as the Numeric Rating Scale (NRS) and Visual Analogue Scale (VAS) provide a single measure to the intensity of (current) pain. More complex questionnaires such as the McGill Pain Questionnaire (MPQ) measure multiple dimensions of pain. Questionnaires targeting a specific patient group exist as well. The Western Ontario and McMaster Universities Osteoarthritis Index (WOMAC) and Knee Society Score (KSS) assign pain, stiffness, and functional scores to patients suffering osteoarthristis. These questionnaires are also used to record and observe patients' healing progress after Total Knee Arthroplasty (TKA) (Hawker, Mian, Kendzerska, \& French, 2011).

While questionnaires are useful for obtaining an overall idea of pain perception and how the pain is affecting patients, it provides little quantification of nociceptive processing. Psychophysical methods aim to describe the relation between physical stimuli and corresponding subjectively reported responses (Kingdom \& Prins, 2009). These methods are widely used to study stimulus processing in fields as vision (Chauhan, Tompkins, LeBlanc, \& McCormick, 1993), audiology (McFadden, 1983), and pain (Sandkühler, 2009). Among the first who related perceptional intensities to stimulus intensities were Weber and Fechner (Fechner, 1860; Weber, 1834). They introduced important terms such as the just-noticeable difference threshold (JND) and detection threshold. A typical example of a psychophysical task is a detection experiment. Subjects are presented stimuli with various amplitudes and are to indicate whether they did or did not detect the stimulus. This type of experiment is relatively easy to set-up and easily understood by subjects.

\section{Relation between stimuli and perceptual detections}

The relation between presented stimuli and responses in a detection experiment is often depicted by a psychometric function. This sigmoidal function relates the detection probability $\psi$ with the stimulus amplitude and is typically described by a threshold $\alpha$ and a slope $\beta$. The threshold $\alpha$ is often defined as the amplitude resulting in a 0.5 detection probability, and thus represents the shift of the function over the amplitude axis. The steepness of the function is depicted by the slope parameter $\beta$ and can be used as a measure of the reliability of stimulus detection by the subject (Gold \& Ding, 2013; Strasburger, 2001). During the experiment, subjects may accidentally lapse or make a 
guess whether they detected the stimulus, possibly affecting the estimation quality of the threshold and slope. Lapses and guesses can be incorporated in the psychometric function as the lapsing rate $\lambda$ and guessing rate $\gamma$ :

$$
\psi(x ; \alpha, \beta, \gamma, \lambda)=\gamma+(1-\gamma-\lambda) F(x ; \alpha, \beta),
$$

where $x$ is the stimulus amplitude and $F(x ; \alpha, \beta)$ a link function describing the sigmoidal form (e.g., cumulative normal, logistic, or Gumbel distribution functions). In case of a logistic function, $F(x ; \alpha, \beta)$ is given by:

$$
F(x ; \alpha, \beta)=\frac{1}{1+\exp (\beta(\alpha-x))}
$$

Combining Equation (1-1) and Equation (1-2) and assuming the lapsing and guessing rates to be zero, the psychometric function (Equation (1-1)) reduces to:

$$
\psi(x ; \alpha, \beta)=\frac{1}{1+\exp (\beta(\alpha-x))} .
$$

Figure 1-1 shows the relation between stimulus amplitude and the detection probability for variations in the threshold $\alpha$ (Figure 1-1A) and slope $\beta$ (Figure 1-1B).

\section{Stimulation of the nociceptive system}

\section{Activation of peripheral nociceptors}

For psychophysical observation of nociceptive mechanisms, stimuli which activate nociceptive mechanisms are required. Out of all modalities used for stimulation of nociceptors, heat stimulation using a $\mathrm{CO}_{2}$ laser is best known for its nociceptive specific stimulation and clinical use. However, due to heat build-up and peripheral sensitization, the inter-stimulus interval is relatively long allowing only one stimulus per 5 to 20 seconds (Mouraux, Iannetti, \& Plaghki, 2010). Accurate control of stimulation timing is possible when using electrical stimulation, allowing well-defined stimuli with relatively high temporal resolutions. However, a disadvantage of electrical stimulation is its non-specificity towards the preferential stimulation of nociceptive fibers. Electrical stimulation of nerve fibers results in substantial co-activation of tactile $A \beta$-fibers and is, therefore, not nociceptive specific. However, several studies have shown that small needle-like electrodes, which slightly protrude through the epidermis, can preferentially activate nociceptive A $\delta$-fibers (Bromm \& Meier, 1984; Inui, Tran, Hoshiyama, \& Kakigi, 


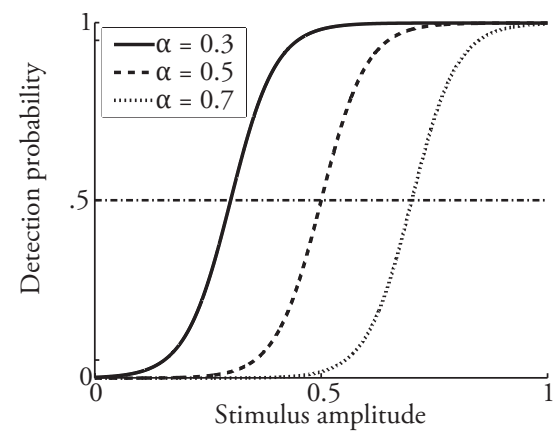

A

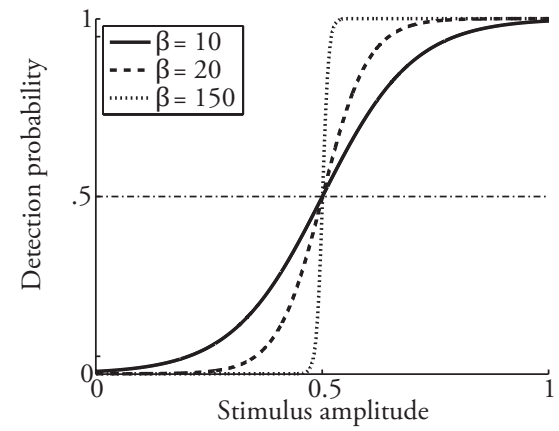

B

Figure 1-1 Psychometric curve using a logistic function denote the detection probability given the stimulus amplitude [a.u.] in a simple detection experiment. (A) A change in threshold $\alpha$ is depicted by a horizontal shift of the psychometric curve. (B) A change in slope $\beta$ is depicted by the steepness of the psychometric curve. Larger values for $\beta$ result in steeper curves.

2002; Mouraux et al., 2010; Nilsson, Levinsson, \& Schouenborg, 1997; Steenbergen, et al, 2012). To minimize the co-activation of A $\beta$-fibers, the stimulation current using intra-epidermal electrical stimulation (IES) should remain below twice the detection threshold (Legrain \& Mouraux, 2013; Mouraux et al., 2010).

Increasing the perceived strength of stimuli can be achieved by increasing the amplitude or pulse-width (PW) of the stimulus. Increasing the amplitude or PW may result in a higher number of activated cutaneous afferents, increasing the induced peripheral neural activity. However, as a result of an increase in the amplitude, stimuli might no longer be nociceptive specific due to an increase in the probability of co-activation of tactile afferents. To increase the perceived strength of stimuli, but also to remain the specificity towards nociceptive stimulation, it was proposed to apply a train of multiple pulses with short inter-pulse intervals (IPI) of less than $200 \mathrm{~ms}$, and relatively low stimulus amplitude. It has been demonstrated that pulse train stimulation increases the neural activity and perceived strength (Mouraux, Marot, \& Legrain, 2014; Steenbergen et al., 2012; van der Heide, Buitenweg, Marani, \& Rutten, 2009). Temporal summation of postsynaptic potentials is a possible explanation for the increased neural activity when stimuli with multiple pulses are presented. Therefore, variations in the stimulus amplitude and PW are expected to affect peripheral activation of cutaneous afferents, and pulse train stimulation and IPI are expected to affect the activation of central processes. 


\section{Activation of descending pain modulation}

For activation of descending pain modulation (e.g., DNIC), or conditioned pain modulation (CPM) (Yarnitsky et al., 2010), a so-called conditioning stimulus is required. Induced cold pain is often used as an experimental method for perturbation of the descending mechanism (Pud, Granovsky, \& Yarnitsky, 2009). For example, the cold pressor test is widely used to study endogenous inhibition (Mitchell, MacDonald, $\&$ Brodie, 2004). During the cold pressor test, subjects immerse an extremity into cold water for a short period of time, which that induces pain. With the help of this painful stimulus, the function of CPM can be observed. As a result of this painful stimulus, $\mathrm{CPM}$ can be studied. Observing the function of CPM is useful for clinical application as it can be used to identify malfunctions in descending pathways (Nir \& Yarnitsky, 2015).

\section{Psychophysical thresholds reflecting nociceptive processing}

Commonly-used methods for observation of nociceptive stimulus processing and sensory function include psychophysical Quantitative Sensory Testing (QST) methods (Arendt-Nielsen \& Yarnitsky, 2009; Rolke et al., 2006). Multiple QST methods exist, using a broad range of stimulus types such as thermal, mechanical, or electrical. QST is used as an indicator of the state of the nociceptive system and can be used to identify nociceptive disease. For example, a decrease in the pain threshold and an increase in pain to suprathreshold stimuli indicates hyperalgesia (Treede, Meyer, Raja, \& Campbell, 1992). Moreover, thresholds are currently thought of as a potentially useful tool for prediction of chronic pain disorders (Backonja et al., 2013; Wilder-Smith et al., 2010). However, as a stimulus is processed by several peripheral and central mechanisms prior to a perception, a single threshold estimate cannot be used to distinguish between these mechanisms. This possible hampers the identification of nociceptive disease and prediction of chronic pain disorders.. Similar to the method presented by Gracely, Lota, Walter, and Dubner (1988), stimuli with various temporal properties can be presented in a random intermingled sequence within a single experiment. The estimated thresholds, corresponding to the various stimulus properties, could help distinguishing the contributions of nociceptive processes to stimulus processing. Moreover, noxious events, such as a conditioning stimulus, activate endogenous analgesic mechanisms (e.g., DNIC) resulting in dynamic changes in nociceptive processing. The effect of a conditioning stimulus is currently estimated by a few pre and post thresholds, unable to capture the dynamic properties of the nociceptive system. Tracking a threshold over time, using a method introduced by von Dincklage, Hackbarth, Schneider, Baars, and Rehberg (2009), helps observing changes nociceptive processing. 


\section{PROBLEM STATEMENT}

Persisting pain occurs frequently after surgical interventions and has major impacts on the quality of life of affected patients. The underlying cause of PPSP is often unclear unknown and, as a result, treatment is relatively ineffective and expensive. Recent studies have suggested a link between nociceptive malfunction and PPSP development and argued that QST is a potentially useful tool for the prediction of PPSP. However, it still remains difficult to distinguish the individual contributions of nociceptive mechanisms, possibly hampering the prediction of PPSP and other pain disorders. A tool allowing the simultaneous observation of individual contributions of peripheral and central mechanisms to stimulus processing could possibly aid in understanding nociceptive mechanisms and the prediction of PPSP.

\section{Context: PaINSIGHT}

The work presented in this thesis is part of the PaINSIGHT project which is part of the NeuroSIPE (system identification and parameter estimation of neurophysiological systems) consortium, supported by the Dutch Technology Foundation STW. This consortium includes several Dutch technical universities, medical universities, and companies. Combining technical and medical techniques, it aims at developing diagnostic techniques for neurological disorders.

The PaINSIGHT project is a collaboration between the University of Twente and University Medical Centre Radboudumc, together with the Centre for Human Drug Research (CHDR) as industrial partner. The Otto Selz Institute of applied psychology of the University of Mannheim and the St. Antonius hospital are involved as users of the study outcomes. The project aim is to design methods which allow observation of the individual involvement of nociceptive mechanisms to stimulus processing and to combine these methods into a clinically applicable tool. The aim is subdivided into two objectives: (1) the development of psychophysical methods allowing observation of nociceptive processing, and (2) the development of computational models describing the nociceptive mechanisms involved in stimulus processing. The work presented in this thesis focuses mainly on the first objective. 


\section{Aims and outline of this thesis}

The primary objective of the work presented in this thesis is to develop a clinically applicable tool for psychophysical observation of nociceptive contributions to sensory processing. Two goals are identified in order to achieve the primary objective:

1. To develop experimental techniques allowing the simultaneous observation of multiple non-stationary psychophysical thresholds.

2. To characterize the contributions of peripheral and central mechanisms to nociceptive sensory processing.

This thesis is subdivided into two parts, each part addressing one of the goals.

Part I focuses on the development of experimental techniques and addresses three methodological issues, each discussed in a separate chapter. Psychophysical methods for tracking of a non-stationary nociceptive threshold over time are compared and discussed in Chapter 2. Chapter 3 deals with time-dependent psychophysical functions and discusses how to account for threshold drifts. The tracking paradigm discussed in Chapter 2 and Chapter 3 is extended in Chapter 4 allowing the simultaneous observation of multiple non-stationary thresholds within a single experiment.

Part II focuses on the application of the experimental techniques presented in Part I and consists of two chapters. In Chapter 5, the effect of temporal stimulus properties on the nociceptive detection probability using intra-epidermal electrical stimulation is studied. Furthermore, it is investigated whether variations in the observed psychophysical function reflect contributions of nociceptive processes. The responsiveness of nociceptive thresholds to capsaicin-induced changes in nociceptive processing is studied in a pilot study described in Chapter 6.

At the end of this thesis, in Chapter 7, all results presented in Chapters 2 to 6 are considered in a general discussion. Moreover, suggestions and an outlook for future experiments are given. 


\section{REFERENCES}

Apfelbaum, J. L., Chen, C., Mehta, S. S., \& Gan, T. J. (2003). Postoperative pain experience: Results from a national survey suggest postoperative pain continues to be undermanaged. Anesthesia and Analgesia, 97(2), 534-540.

Arendt-Nielsen, L., \& Yarnitsky, D. (2009). Experimental and Clinical Applications of Quantitative Sensory Testing Applied to Skin, Muscles and Viscera. Journal of Pain, 10(6), 556-572.

Backonja, M. M., Attal, N., Baron, R., Bouhassira, D., Drangholt, M., Dyck, P. J., . . Ziegler, D. (2013). Value of quantitative sensory testing in neurological and pain disorders: NeuPSIG consensus. Pain, 154(9), 1807-1819.

Bekkering, G. E., Bala, M. M., Reid, K., Kellen, E., Harker, J., Riemsma, R., . . Kleijnen, J. (2011). Epidemiology of chronic pain and its treatment in The Netherlands. Netherlands Journal of Medicine, 69(3), 141-153.

Bromm, B., \& Lorenz, J. (1998). Neurophysiological evaluation of pain. Electroencephalography and Clinical Neurophysiology, 107(4), 227-253.

Bromm, B., \& Meier, W. (1984). The intracutaneous stimulus: a new pain model for algesimetric studies. Methods and Findings in Experimental and Clinical Pharmacology, 6(7), 405-410.

Chauhan, B. C., Tompkins, J. D., LeBlanc, R. P., \& McCormick, T. A. (1993). Characteristics of frequency-of-seeing curves in normal subjects, patients with suspected glaucoma, and patients with glaucoma. Investigative Ophthalmology and Visual Science, 34(13), 3534-3540.

Crombie, I. K., Davies, H. T., \& Macrae, W. A. (1998). Cut and thrust: antecedent surgery and trauma among patients attending a chronic pain clinic. Pain, 76(1-2), 167-171.

D'Apuzzo, M. R., Cabanela, M. E., Trousdale, R. T., \& Sierra, R. J. (2012). Primary total knee arthroplasty in patients with fibromyalgia. Orthopedics, 35(2), e175-178.

D'Mello, R., \& Dickenson, A. H. (2008). Spinal cord mechanisms of pain. British Journal of Anaesthesia, 101(1), 8-16.

Daneshjou, K., Jafarieh, H., \& Raaeskarami, S.-R. (2012). Congenital Insensitivity to Pain and Anhydrosis (CIPA) Syndrome; A Report of 4 Cases. Iranian Journal of Pediatrics, 22(3), 412-416.

Dubin, A. E., \& Patapoutian, A. (2010). Nociceptors: The sensors of the pain pathway. Journal of Clinical Investigation, 120(11), 3760-3772.

Edwards, R. R., Doleys, D. M., Fillingim, R. B., \& Lowery, D. (2001). Ethnic differences in pain tolerance: clinical implications in a chronic pain population. Psychosomatic Medicine, 63(2), 316-323.

Fechner, G. T. (1860). Elemente der psychophysik. Leipzig,: Breitkopf und Härtel.

Gold, J. I., \& Ding, L. (2013). How mechanisms of perceptual decision-making affect the psychometric function. Progress in Neurobiology, 103, 98-114.

Gracely, R. H., Lota, L., Walter, D. J., \& Dubner, R. (1988). A multiple random staircase method of psychophysical pain assessment. Pain, 32(1), 55-63.

Harstall, C., \& Ospina, M. (2003). How prevalent is chronic pain? Pain: Clinical Updates, 11(2).

Hawker, G. A., Mian, S., Kendzerska, T., \& French, M. (2011). Measures of adult pain: Visual Analog Scale for Pain (VAS Pain), Numeric Rating Scale for Pain (NRS Pain), McGill Pain Questionnaire (MPQ), Short-Form McGill Pain Questionnaire (SF-MPQ), Chronic Pain Grade Scale (CPGS), Short Form-36 Bodily Pain Scale (SF-36 BPS), and Measure of Intermittent and Constant Osteoarthritis Pain (ICOAP). Arthritis Care and Research, 63(SUPPL. 11), S240-S252.

Hunfeld, J. A., Perquin, C. W., Duivenvoorden, H. J., Hazebroek-Kampschreur, A. A., Passchier, J., van Suijlekom-Smit, L. W., \& van der Wouden, J. C. (2001). Chronic pain and its impact on quality of life in adolescents and their families. Journal of Pediatric Psychology, 26(3), 145-153.

International Association for the Study of Pain (IASP). (1994). Classification of Chronic Pain.

International Association for the Study of Pain (IASP), C. o. T. (2011). Part III: Pain Terms, A Current List with Definitions and Notes on Usage.

Inui, K., Tran, T. D., Hoshiyama, M., \& Kakigi, R. (2002). Preferential stimulation of A $\delta$ fibers by intra-epidermal needle electrode in humans. Pain, 96(3), 247-252. 
Keefe, F. J., Lumley, M., Anderson, T., Lynch, T., \& Carson, K. L. (2001). Pain and emotion: New research directions. Journal of Clinical Psychology, 57(4), 587-607.

Kehlet, H., Jensen, T. S., \& Woolf, C. J. (2006). Persistent postsurgical pain: risk factors and prevention. Lancet, 367(9522), 1618-1625.

Kingdom, F. A. A., \& Prins, N. (2009). Psychophysics: A Practical Introduction: Elsevier Science \& Technology.

Latremoliere, A., \& Woolf, C. J. (2009). Central Sensitization: A Generator of Pain Hypersensitivity by Central Neural Plasticity. Journal of Pain, 10(9), 895-926.

Le Bars, D. (2002). The whole body receptive field of dorsal horn multireceptive neurones. Brain Research Reviews, 40(1-3), 29-44.

Le Bars, D., Dickenson, A. H., \& Besson, J. M. (1979a). Diffuse noxious inhibitory controls (DNIC). I. Effects on dorsal horn convergent neurones in the rat. Pain, 6(3), 283-304.

Le Bars, D., Dickenson, A. H., \& Besson, J. M. (1979b). Diffuse noxious inhibitory controls (DNIC). II. Lack of effect on non-convergent neurones, supraspinal involvement and theoretical implications. Pain, 6(3), 305-327.

Legrain, V., \& Mouraux, A. (2013). Activating selectively and reliably nociceptive afferents with concentric electrode stimulation: Yes we can! Provided that low stimulus intensities are used! Clinical Neurophysiology, 124(2), 424.

Li, J., Simone, D. A., \& Larson, A. A. (1999). Windup leads to characteristics of central sensitization. Pain, 79(1), $75-82$.

Macrae, W. A. (2001). Chronic pain after surgery. British Journal of Anaesthesia, 87(1), 88-98.

Macrae, W. A. (2008). Chronic post-surgical pain: 10 years on. British Journal of Anaesthesia, 101(1), 77-86.

McFadden, D. F. L. (1983). Audition: Some relations between normal and pathalogical hearing. Annual Review of Psychology, 34(1), 95.

Melzack, R., \& Wall, P. D. (1965). Pain mechanisms: A new theory. Science, 150(3699), 971-979.

Mendell, L. M. (2011). Computational functions of neurons and circuits signaling injury: Relationship to pain behavior. Proceedings of the National Academy of Sciences of the United States of America, 108(SUPPL. 3), 15596-15601.

Millan, M. J. (1999). The induction of pain: An integrative review. Progress in Neurobiology, 57(1), 1-164.

Mitchell, L. A., MacDonald, R. A., \& Brodie, E. E. (2004). Temperature and the cold pressor test. Journal of Pain, 5(4), 233-237.

Moayedi, M., \& Davis, K. D. (2013). Theories of pain: From specificity to gate control. Journal of Neurophysiology, $109(1), 5-12$.

Mogil, J. S. (2012). Sex differences in pain and pain inhibition: multiple explanations of a controversial phenomenon. Nature Reviews: Neuroscience, 13(12), 859-866.

Mouraux, A., Iannetti, G. D., \& Plaghki, L. (2010). Low intensity intra-epidermal electrical stimulation can activate A $\delta$-nociceptors selectively. Pain, 150(1), 199-207.

Mouraux, A., Marot, E., \& Legrain, V. (2014). Short trains of intra-epidermal electrical stimulation to elicit reliable behavioral and electrophysiological responses to the selective activation of nociceptors in humans. Neuroscience Letters, 561, 69-73.

Nagasako, E. M., Oaklander, A. L., \& Dworkin, R. H. (2003). Congenital insensitivity to pain: an update. Pain, 101(3), 213-219.

Nilsson, H. J., Levinsson, A., \& Schouenborg, J. (1997). Cutaneous field stimulation (CFS): A new powerful method to combat itch. Pain, 71(1), 49-55.

Nir, R. R., \& Yarnitsky, D. (2015). Conditioned pain modulation. Current Opinion in Supportive and Palliative Care, 9(2), 131-137.

Perkins, F. M., \& Kehlet, H. (2000). Chronic pain as an outcome of surgery. A review of predictive factors. Anesthesiology, 93(4), 1123-1133.

Pud, D., Granovsky, Y., \& Yarnitsky, D. (2009). The methodology of experimentally induced diffuse noxious inhibitory control (DNIC)-like effect in humans. Pain, 144(1-2), 16-19.

Purves, D., Augustine, G. J., Fitzpatrick, D., Hall, W. C., LaMantia, A. S., McNamara, J. O., \& White, L. E. (2008). Neuroscience. Sunderland, MA: Sinauer Associates, Inc. 
Rolke, R., Baron, R., Maier, C., Tölle, T. R., Treede, R. D., Beyer, A., . . Wasserka, B. (2006). Quantitative sensory testing in the German Research Network on Neuropathic Pain (DFNS): Standardized protocol and reference values. Pain, 123(3), 231-243.

Saarto, T., \& Wiffen, P. J. (2007). Antidepressants for neuropathic pain. Cochrane Database Syst Rev (4), CD005454.

Sandkühler, J. (2009). Models and mechanisms of hyperalgesia and allodynia. Physiological Reviews, 89(2), 707-758.

Schaible, H. G. (2007). Peripheral and central mechanisms of pain generation. Handbook of Experimental Pharmacology (177), 3-28.

Steenbergen, P., Buitenweg, J. R., Trojan, J., van der Heide, E. M., van den Heuvel, T., Flor, H., \& Veltink, P. H. (2012). A system for inducing concurrent tactile and nociceptive sensations at the same site using electrocutaneous stimulation. Behavior Research Methods, 44(4), 924-933.

Strasburger, H. (2001). Converting between measures of slope of the psychometric function. Perception and Psychophysics, 63(8), 1348-1355.

Todd, A. J. (2010). Neuronal circuitry for pain processing in the dorsal horn. Nature reviews. Neuroscience, 11(12), 823-836.

Treede, R. D., Meyer, R. A., Raja, S. N., \& Campbell, J. N. (1992). Peripheral and central mechanisms of cutaneous hyperalgesia. Progress in Neurobiology, 38(4), 397-421.

Treede, R. D., Rief, W., Barke, A., Aziz, Q., Bennett, M. I., Benoliel, R., . . . Wang, S. J. (2015). A classification of chronic pain for ICD-11. Pain, 156(6), 1003-1007.

Tsang, A., Von Korff, M., Lee, S., Alonso, J., Karam, E., Angermeyer, M. C., . . Watanabe, M. (2008). Common chronic pain conditions in developed and developing countries: gender and age differences and comorbidity with depression-anxiety disorders. Journal of Pain, 9(10), 883-891.

van der Heide, E. M., Buitenweg, J. R., Marani, E., \& Rutten, W. L. (2009). Single pulse and pulse train modulation of cutaneous electrical stimulation: a comparison of methods. Journal of Clinical Neurophysiology, 26(1), 54-60.

von Dincklage, F., Hackbarth, M., Schneider, M., Baars, J. H., \& Rehberg, B. (2009). Introduction of a continual RIII reflex threshold tracking algorithm. Brain Research, 1260, 24-29.

Wall, P. D., \& Melzack, R. (1999). Textbook of pain. London: Churchill Livingston.

Washington, L. L., Gibson, S. J., \& Helme, R. D. (2000). Age-related differences in the endogenous analgesic response to repeated cold water immersion in human volunteers. Pain, 89(1), 89-96.

Weber, E. H. (1834). De Pulsu, resoptione, auditu et tactu. Annotationes anatomicae et physiologicae. Lipsiae: C.F. Koehler.

West, S. J., Bannister, K., Dickenson, A. H., \& Bennett, D. L. (2015). Circuitry and plasticity of the dorsal horn Toward a better understanding of neuropathic pain. Neuroscience, 300, 254-275.

Wiffen, P. J., McQuay, H. J., Edwards, J. E., \& Moore, R. A. (2005). Gabapentin for acute and chronic pain. Cochrane Database Syst Rev (3), CD005452.

Wilder-Smith, O. H., \& Arendt-Nielsen, L. (2006). Postoperative hyperalgesia: its clinical importance and relevance. Anesthesiology, 104(3), 601-607.

Wilder-Smith, O. H., Schreyer, T., Scheffer, G. J., \& Arendt-Nielsen, L. (2010). Patients with chronic pain after abdominal surgery show less preoperative endogenous pain inhibition and more postoperative hyperalgesia: a pilot study. Journal of Pain \& Palliative Care Pharmacotherapy, 24(2), 119-128.

Woolf, C. J. (2011). Central sensitization: Implications for the diagnosis and treatment of pain. Pain, 152(SUPPL.3), S2-S15.

Woolf, C. J., \& Ma, Q. (2007). Nociceptors-Noxious Stimulus Detectors. Neuron, 55(3), 353-364.

Woolf, C. J., \& Salter, M. W. (2000). Neuronal plasticity: increasing the gain in pain. Science, 288(5472), 1765-1769.

Yarnitsky, D., Arendt-Nielsen, L., Bouhassira, D., Edwards, R. R., Fillingim, R. B., Granot, M., ... Wilder-Smith, O. (2010). Recommendations on terminology and practice of psychophysical DNIC testing. European Journal of Pain (London, England), 14(4), 339. 

Psychophysical methods

for observation

of sensory function

\section{Part I}





\section{Chapter 2}

Tracking of nociceptive thresholds

using adaptive psychophysical procedures

Robert J. Doll, Jan R. Buitenweg, Hil G.E. Meijer, Peter H. Veltink

Behavior Research Methods

$(2014) 46(1), 55-66$

DOI $10.3758 / \mathrm{s} 13428-013-0368-4$ 
Abstract Psychophysical thresholds reflect the state of the underlying nociceptive mechanisms. For example, noxious events can activate endogenous analgesic mechanisms that increase the nociceptive threshold. Therefore, tracking thresholds over time facilitates the investigation of the dynamics of these underlying mechanisms. Threshold tracking techniques should use efficient methods for stimulus selection and threshold estimation. This study compares, in simulation and in human psychophysical experiments, the performance of different combinations of adaptive stimulus selection procedures and threshold estimation methods. Monte Carlo simulations were first performed to compare the bias and precision of threshold estimates produced by three different stimulus selection procedures (simple staircase, random staircase, and minimum entropy procedure) and two estimation methods (logistic regression and Bayesian estimation). Logistic regression and Bayesian estimations resulted in similar precision only when the prior probability distributions (PDs) were chosen appropriately. The minimum entropy and simple staircase procedures achieved the highest precision, while the random staircase procedure was the least sensitive to different procedure specific settings. Next, the simple staircase and random staircase procedures, in combination with logistic regression, were compared in a human subject study $(\mathrm{n}=30)$. Electrocutaneous stimulation was used to track the nociceptive perception threshold before, during, and after a cold pressor task, which served as the conditioning stimulus. With both procedures, habituation was detected, as well as changes induced by the conditioning stimulus. However, the random staircase procedure achieved a higher precision. We recommend using the random staircase over the simple staircase procedure, in combination with logistic regression, for non-stationary threshold tracking experiments. 


\section{INTRODUCTION}

Noxious events such as disease (e.g., hyperalgesia), clinical interventions (Wilder-Smith, Schreyer, Scheffer, \& Arendt-Nielsen, 2010; Yarnitsky et al., 2008), and experimental conditioning stimuli (e.g., cold pressor task; see Pud, Granovsky, \& Yarnitsky, 2009) can activate endogenous analgesic mechanisms, which will, in turn, raise the nociceptive thresholds. Tracking psychophysical thresholds over time thus facilitates the investigation of the dynamics of the underlying mechanisms, which can be useful, for example, in identifying patients with an increased risk of developing postsurgical chronic pain (Wilder-Smith et al., 2010; Yarnitsky et al., 2008; Yarnitsky, Granot, Nahman-Averbuch, Khamaisi, \& Granovsky, 2012).

Stationary thresholds can be estimated using psychophysical paradigms such as forced choice tasks, signal detection approaches, and yes-no experiments (Kingdom \& Prins, 2009). In yes-no experiments, multiple stimulus amplitudes and their corresponding responses (i.e., perceived or not) are used to probe the subjects' psychophysical function (Gescheider, 1985; Kingdom \& Prins, 2009; Klein, 2001; Treutwein, 1995). Threshold measurements therefore include (1) stimulus selection procedures for the collection of stimulus-response pairs and (2) estimation methods for determining the most likely threshold from the collected stimulus-response pairs.

Stimulus selection procedures can be either adaptive or nonadaptive. In nonadaptive procedures, new stimuli are selected independently of the preceding stimulus-response pairs. For example, the method of constant stimuli (e.g., Simpson, 1988) involves selecting stimulus amplitudes randomly from a set of predefined values, thus allowing a global probing of the psychophysical function. However, due to the fixed range of predefined amplitudes and a relatively large number of required stimuli, these procedures are inefficient, as compared with adaptive procedures (Watson \& Fitzhugh, 1990).

Adaptive procedures select new stimuli on the basis of preceding stimulus-response pairs and have been demonstrated to be more efficient than non adaptive procedures (e.g., Leek, 2001). A widely used adaptive stimulus selection procedure is the simple up-down staircase procedure (e.g., Cornsweet, 1962), during which the stimulus amplitude is increased after a negative response to the preceding stimulus and decreased after a positive response. In this way, one can probe around the threshold of the psychophysical function. However, subjects may be able to identify and, therefore, anticipate the sequential order of the stimuli (Ehrenstein \& Ehrenstein, 1999; Levitt, 1971). 
A more advanced stimulus selection procedure is to select the stimuli that will minimize the expected entropy of the posterior $\mathrm{PD}$ and will, therefore, provide maximum information about the psychophysical curve (Kontsevich \& Tyler, 1999; Kujala \& Lukka, 2006). This minimum entropy procedure requires a prior PD, which is continuously updated with new stimulus-response pairs according to Bayes rule. Due to this requirement, assumptions must be made about the possible ranges of the psychophysical parameters (e.g., threshold). When little information is available about these parameters, broad prior PDs might be necessary at the expense of increased computational load (Kingdom \& Prins, 2009; Kontsevich \& Tyler, 1999; Kujala \& Lukka, 2006).

Since stimulus responses are binary in the "yes-no" paradigm, signifying either perceived or not perceived, logistic regression can be used to estimate the threshold (e.g., Hosmer\&Lemeshow, 2000). Alternatively, the results of each subsequent stimulus-response pair can be used to estimate the posterior PD according to Bayes rule (e.g., King-Smith \& Rose, 1997; Kontsevich \& Tyler, 1999; Kujala \& Lukka, 2006; Treutwein \& Strasburger, 1999; Watson \& Pelli, 1983).

For tracking thresholds over time, a moving time window, which includes only the most recent stimulus-response pairs, can be used (von Dincklage, Hackbarth, Schneider, Baars, \& Rehberg, 2009). The length of this time window is determined by (1) the number of stimulus-response pairs used for threshold estimation and (2) the interstimulus intervals (i.e., the time subjects require to indicate whether stimuli are perceived). To effectively track non-stationary thresholds over time, this time window should be sufficiently small. However, this will limit the number of stimulus-response pairs available for momentary threshold estimations. Fewer stimulus-response pairs will, in turn, result in higher estimation bias and lower estimation precision. Therefore, efficient stimulus selection procedures and threshold estimation methods are crucial for successful threshold tracking.

We introduce a new adaptive stimulus selection procedure, which we refer to as the random staircase procedure, which overcomes the disadvantages of the procedures described above. Stimuli are randomly selected from a small, predefined set of amplitudes (as is the case with the method of constant stimuli). All amplitudes in the set are increased by a fixed step size after a not-perceived stimulus and decreased after a perceived stimulus (as with the simple staircase procedure). In this way, the high computational load of the minimum entropy procedure will be avoided, as will the high stimulus predictability of the simple staircase procedure. 
Although comparisons between different combinations of stimulus selection procedures and estimation methods have been widely conducted for stationary thresholds, their performances in determining non-stationary thresholds over longer periods of time have not been extensively studied. Therefore, the aim of this study is to compare the performance of various combinations of adaptive stimulus selection procedures and threshold estimation methods for the use in psychophysical threshold tracking experiments.

We performed Monte Carlo simulations of a modeled psychophysical experiment to compare three adaptive stimulus selection procedures and two threshold estimation methods in terms of the bias and precision of their threshold estimateswith both stationary and non-stationary thresholds. Subsequently, a human subject study was conducted to compare two stimulus selection procedures for threshold tracking in a human subject study where we tracked nociceptive perception thresholds using electrocutaneous stimulation and employed a cold pressor task to perturb the nociceptive system.

\section{MONTE CARLO SIMULATIONS}

\section{Method}

The bias and precision of three adaptive stimulus selection procedures and two estimation methodswerecompared by means of MonteCarlo simulations. Astochasticpsychophysical model was used to simulate the responses to stimuli. New stimulus amplitudes were selected according to the stimulus selection procedures. The simulated stimulus-response pairs were used in the estimation methods to generate threshold estimates, which were then compared with the true threshold to obtain their bias and precision.

\section{Psychophysical model}

The probability $p$ of detecting a stimulus of amplitude $x[\mathrm{~mA}]$ was modeled with a logistic psychometric function:

$$
p(x)=(1+\exp (\beta(\alpha-x)))^{-1},
$$

where the slope parameter $\beta$ of the logistic function was fixed at $\beta=20[\mathrm{~mA}]^{-1}$ and the stationary threshold at $\alpha=0.3 \mathrm{~mA}$. Both the lapsing and guessing rates were assumed to be zero and were therefore not included in Equation (2-1). Responses to a stimulus of amplitude $x$ were classified as perceived if $p(x)>\varepsilon$, where $\varepsilon$ is a random number drawn from a uniform distribution between 0 and 1 , and as not perceived otherwise. The random number generator was shuffled each time a new simulation began. 


\section{Simple staircase stimulus selection}

The simple staircase procedure generated new stimuli on the basis of the response to the preceding stimulus: The stimulus amplitude was increased or decreased with the same step size when the previous stimulus was not-perceived or perceived, respectively. The initial stimulus amplitude was set to zero, and a fixed step size of $0.05,0.1$, or $0.2 \mathrm{~mA}$ was used for both the increment and decrement steps.

\section{Random staircase stimulus selection}

The random staircase procedure started with a predefined set of equidistant stimulus amplitudes between 0 and $0.3 \mathrm{~mA}$, from which new stimulus amplitudes were randomly selected. The number of amplitudes (NoA) in the set was either 5 or 10; thus, the amplitudes were separated by 0.075 or $0.033 \mathrm{~mA}$, respectively. All amplitudes in the set were increased by the same step size $(0.05,0.1$, or $0.2 \mathrm{~mA})$ after a not-perceived stimulus and decreased after a perceived stimulus.

\section{Minimum entropy stimulus selection}

The minimum entropy procedure used in this study was based on the procedure described by Kontsevich and Tyler (1999). New stimulus amplitudes were chosen such that they would minimize the expected entropy of the posterior probability density distribution of the psychophysical parameters. Various prior distributions were used (see Table 2-1 for details).

\section{Threshold estimation methods}

Two estimation methods were compared in the simulations: logistic regression (Hosmer\&Lemeshow, 2000) and Bayesian estimation (Harvey, 1986; King-Smith \& Rose, 1997; Kingdom \& Prins, 2009; Treutwein, 1995, 1997; Treutwein, Rentschler, \& Caelli, 1989; Watson \& Pelli, 1983). A logistic function (Equation (2-1)) was used in the Bayesian estimationmethod to model the conditional probability of the response to different stimulus amplitudes. As is indicated in Table 2-1, several different PDs were

Table 2-1 Settings used for the probability distribution (PD): the range of the threshold $\alpha$, slope parameter $\log (\beta)$, and stimulus amplitude $x$ were varied.

\begin{tabular}{|c|c|c|c|c|c|c|c|c|c|}
\hline \multirow[t]{2}{*}{ PD setting (\#) } & \multicolumn{3}{|c|}{ Threshold $\alpha$ range [mA] } & \multicolumn{3}{|c|}{ Slope $\log (\beta)$ range $\left[\mathrm{mA}^{-1}\right]$} & \multicolumn{3}{|c|}{ Amplitude $x$ range $[\mathrm{mA}]$} \\
\hline & Min & Step & $\operatorname{Max}$ & Min & Step & $\operatorname{Max}$ & Min & Step & Max \\
\hline 1 & 0 & 0.01 & 1 & 0.05 & 0.1475 & 3 & 0 & 0.01 & 1 \\
\hline 2 & 0 & 0.01 & 2 & 0.05 & 0.1475 & 3 & 0 & 0.01 & 2 \\
\hline 3 & 0 & 0.015 & 1 & 2.3 & 0.0925 & 6 & 0 & 0.015 & 1 \\
\hline
\end{tabular}




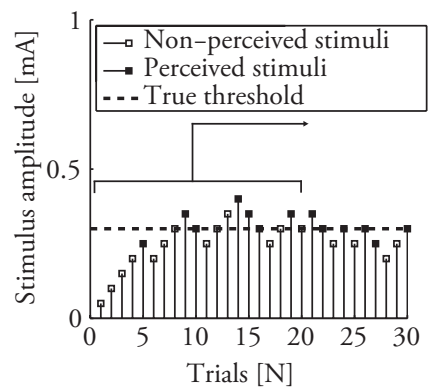

A

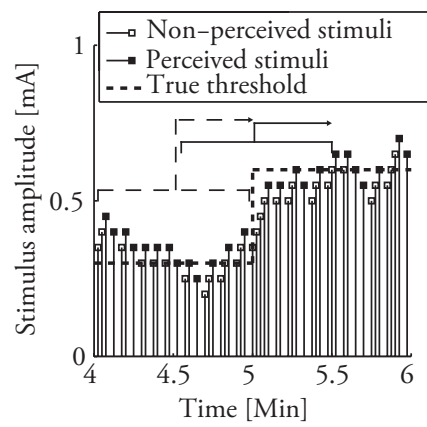

D

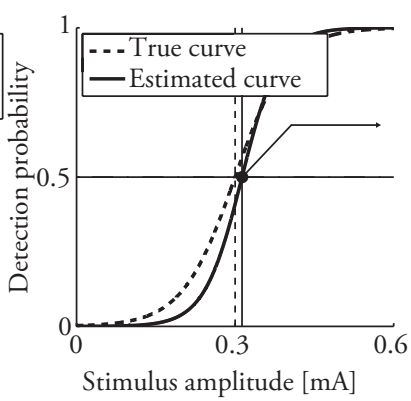

B

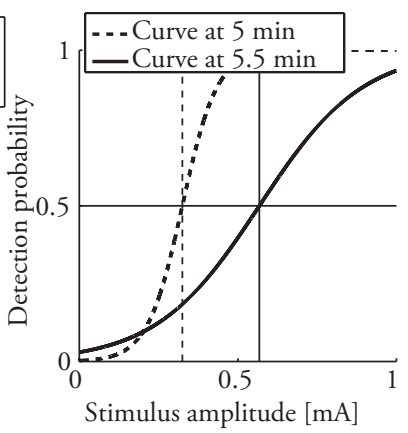

E

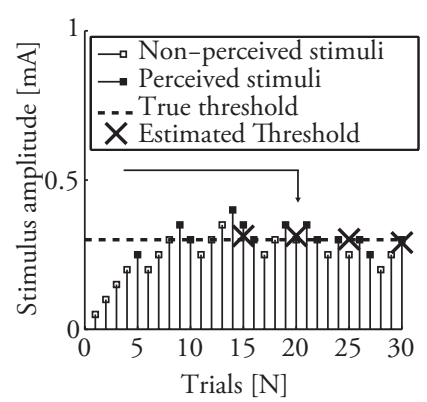

C

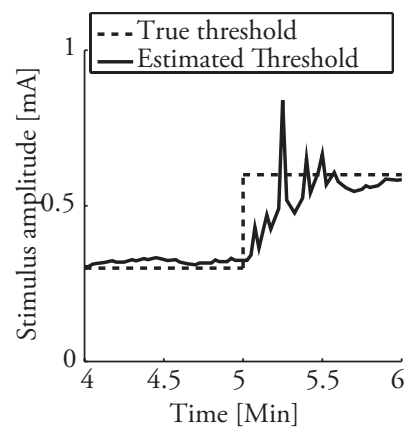

F

Figure 2-1 Schematic procedures for the simulations by which different stimulus selection procedures and threshold estimation methods are compared. A - C Stationary threshold simulations: a window including $N$ stimulus-response paires (A) is used in estimation methods to obtain the threshold estimate (B). (C) A single simulated experiment. D - F Non-stationary threshold simulations: a moving window including 25 stimulus-response pairs (D) is used to estimate (E) and track (F) thresholds over time.

used in the simulations, each with a different set of ranges and sampling resolutions for the threshold $\alpha$, the slope parameter $\beta$, and the stimulus amplitude $x$. Note that the slope parameter $\beta$ was defined in natural $\log$ units. The parameter ranges in PD \#1 and $\mathrm{PD} \# 2$ were chosen such that the true slope parameter $\beta$ was at the upper limit of the slope range. The settings of $\mathrm{PD} \# 3$ were chosen such that the true slope parameter $\beta$ was in the middle of the slope range. A uniform a priori PD was assumed at the start of each simulation.

\section{Simulations}

Three adaptive stimulus selection procedures, in combination with two different estimation methods, were compared in two situations, one with a stationary threshold $\alpha$ (Figure 2-1A-C), and the other with a non-stationary threshold $\alpha$ (Figure 2-1D-F). Stationary thresholds were estimated on the basis of $15,20,25$, or 30 stimulus-response pairs (Figure 2-1C), and each simulation was repeated 5,000 times. The non-stationary threshold situation was used to simulate a 10-min psychophysical experiment in which 
Simple staircase -Bayesian, step size $=0.1$, PD\# 1 Bayesian, step size $=0.1, \mathrm{PD} \# 3$ 口Log. reg., step size $=0.05$ Log. reg., step size $=0.1$ 口Log. reg., step size $=0.2$

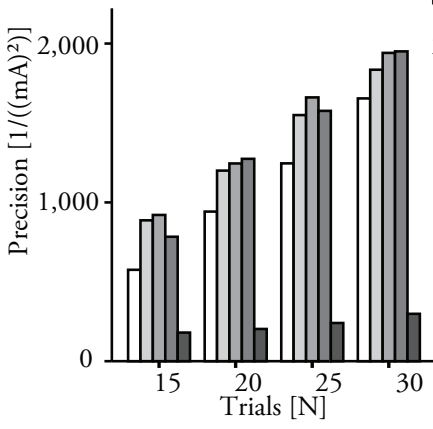

A
Random staircase

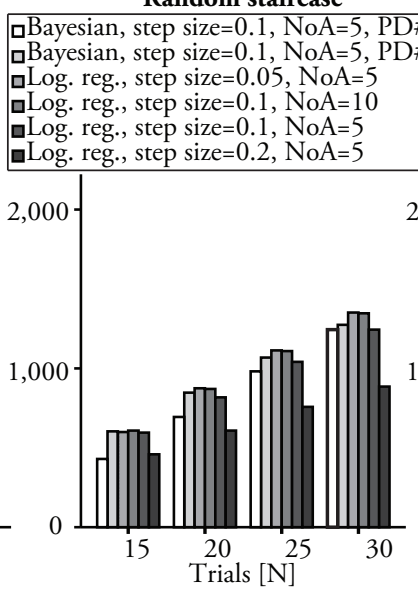

B
Minimum entropy

םBayesian, PD\#1

Log. reg., PD\#1

Bayesian, PD\#2

LLog. reg.,PD\#2

Bayesian, PD\#3

Log. reg., PD\#3

更

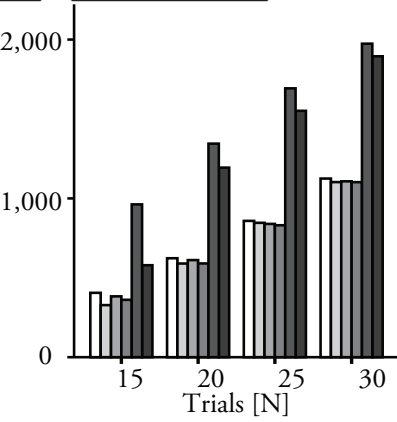

C

Figure 2-2 Precision values of the simulated adaptive procedures with different settings for the three selection procedures. (A) Precision values of the simple staircase procedure: step size, estimation method, and probability distribution (PD) settings (Table 2-1) are varied. (B) Precision values of the random staircase procedure: step size, number of amplitudes (NoA), estimation method, and PD number are varied. (C) Precision values of the minimum entropy procedure: PD settings are varied.

the threshold was held at $0.3 \mathrm{~mA}$ during the first $5 \mathrm{~min}$ and $0.6 \mathrm{~mA}$ for the last $5 \mathrm{~min}$. To see how bias and precision were affected over time by a transient in true threshold, interstimulus intervals of 1.5 and $3.5 \mathrm{~s}$ were included after a not perceived stimulus and a perceived stimulus, respectively, in correspondence with our experimental paradigm. The momentary threshold was estimated using the preceding 25 stimulus-response pairs, and for Bayesian estimations, starting from a uniform prior distribution. We remark that using all previous stimulus-response pairs in a non-stationary simulation results in poor tracking of changes in thresholds (results are not shown). Each simulation was repeated 1,000 times.

\section{Analysis}

The bias and precision of the threshold estimates were determined in all simulations (Treutwein, 1995). Bias was defined as the mean difference between the true and the estimated thresholds, and precision as the reciprocal of the variance of the threshold estimates. To produce equally spaced estimates, non-stationary results were linearly interpolated using a rate of $1 \mathrm{~Hz}$ to prevent undersampling. All simulation models and analyses were performed with MATLAB 7.14 (MathWorks, Inc., Natick, MA). 


\section{RESULTS}

\section{Stationary threshold}

The bias for the stationary threshold situation was found to be lower than $5 \%$ of the true threshold for all but two simulations-namely, when Bayesian estimation (PD \#1) was combined with the simple staircase procedure (step size $=0.1 \mathrm{~mA}, \mathrm{~N} \leq 30$ ) and with the random staircase procedure (step size $=0.1 \mathrm{~mA}, \mathrm{NoA}=5, \mathrm{~N}=15$ ).

Precision values for the stationary threshold situation are presented in Figure 2-2. Overall, precision increased when more stimulus-response pairs were included (Figure 2-2A-C). The estimation precision of logistic regression was higher than or similar to that of Bayesian estimation for both the simple and random staircase procedures. On the other hand, the estimation precision of the minimum entropy procedure was slightly higher when combined with Bayesian estimation than with logistic regression.

The simple staircase procedure (Figure 2-2A) showed higher estimation precision with smaller step sizes, while the precision of the random staircase procedure (Figure 2-2B) was mostly independent of the various settings (e.g., step size, NoA, and estimation method). The minimum entropy procedure (Figure 2-2C) had a higher precision when the true slope parameter $\beta$ was in the middle of the PD (i.e., when PD \#3 was used) than when it was at the upper limit of the PD (i.e., when PD \#1 and PD \#2 were used, respectively).

The estimation precision of the minimum entropy procedure ( $\mathrm{PD} \# 3$ ), in combination with a Bayesian estimation method, was found to be higher than that of the random staircase procedure but similar to that of the simple staircase procedure. However, the precision of the minimum entropy procedure with the slope at the upper limit (PD \#1 and PD \#2) was found to be lower than the other procedures, except when the step size in the two staircase procedures was relatively large (i.e., step size $=0.2$ ).

\section{Non-stationary threshold}

On the basis of the results of the stationary threshold simulations, we chose to simulate non-stationary thresholds with only two settings for the simple staircase procedure: step size $=0.05$ and $0.1 \mathrm{~mA}$. Three settings were chosen for the random staircase procedure: (1) step size $=0.05 \mathrm{~mA}$ and $\mathrm{NoA}=5$, (2) step size $=0.1 \mathrm{~mA}$ and $\mathrm{NoA}=10$, and (3) step size $=0.1 \mathrm{~mA}$ and $\mathrm{NoA}=5 . \mathrm{PD} \# 1$ and $\mathrm{PD} \# 3$ were chosen for the minimum entropy procedure. 

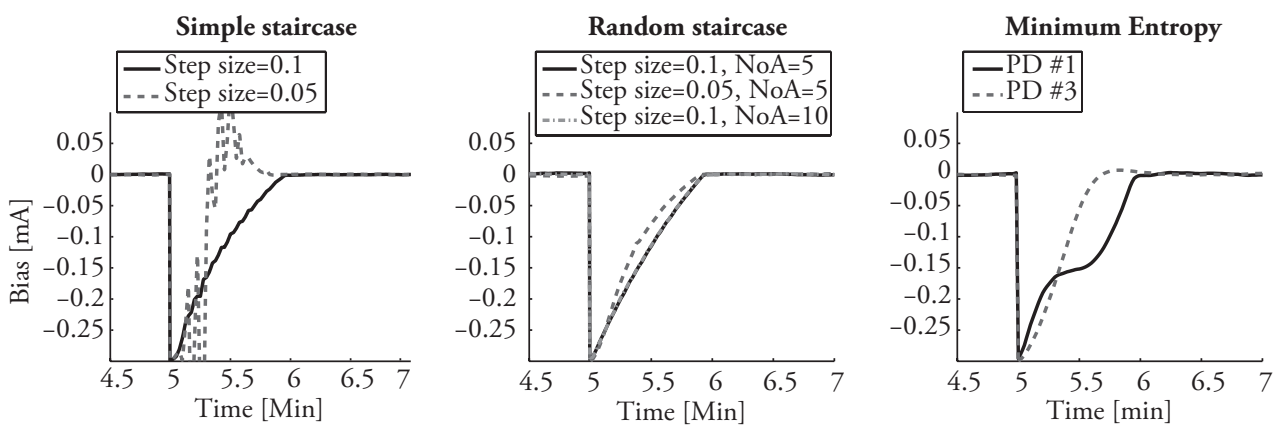

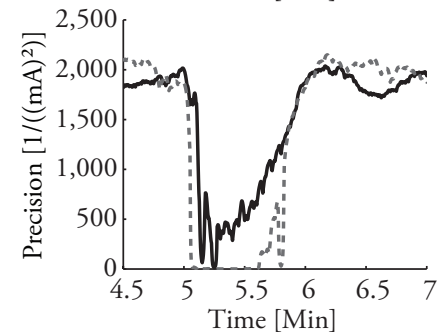

A

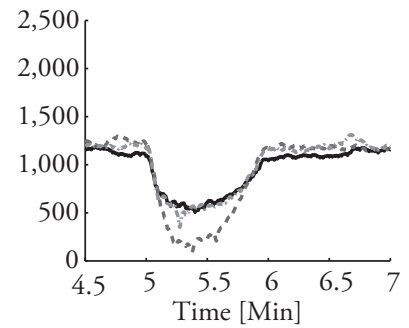

B

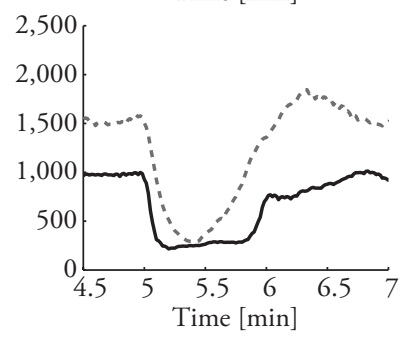

$\mathrm{C}$

Figure 2-3 Bias (top) and precision (bottom) over time of simulated adaptive procedures with different settings. Bias was defined as the mean difference between the true and the estimated thresholds, and precision was defined as the reciprocal of the variance of threshold estimates. Note that the figures display only the results of the simulations between 4.5 and 7 min. Bias and precision of $(\mathbf{A})$ the simple staircase procedure, $(\mathbf{B})$ the random staircase procedure, and $(\mathbf{C})$ the minimum entropy procedure are shown.

Figure 2-3 shows the bias and precision over time around the point of change in the threshold (namely, between 4.5 and $7 \mathrm{~min}$ ). The upper and lower graphs illustrate the bias and precision, respectively. In all simulations, the bias was negligible before the point of change in threshold. Moreover, all combinations of procedures and estimation methods needed a similar amount of time to converge to a negligible bias after the point of change in the threshold. The bias of the simple staircase procedure (step size $=0.05$ ) showed over and undershoot after the point of change in threshold. This was due to the very small step size in comparison with the change in threshold.

Precision decreased after the point of change in the threshold for all procedures. For the simple staircase procedure, the precision was similar for both settings before the point of change in threshold. However, after the change, the precision was drastically reduced when a smaller step size was used. That the precision tended to go near zero implied relatively large variances in the estimations. This was due to the step size being small, as compared with the change in threshold. For the random staircase procedure, the precision was similar for all settings before the point of change in threshold. Again, a smaller step size resulted in a lower precision after the point of change. The minimum 
Figure 2-4 The compound electrode used in this study. The electrode consists of five needles and four flat electrodes.

entropy procedure had a higher precision when the true slope parameter $\beta$ was in the middle of the PD (PD \#3) than when it was at the upper limit (PD \#1 and PD \#2).

Before the point of change in the threshold, the simple staircase procedure produced threshold estimates with the highest precision, as compared with the other procedures. In addition, both the simple and random staircase procedures with a step size of 0.1 $\mathrm{mA}$ had higher precision than the minimum entropy procedure with PDs \#1 and \#2. Nevertheless, the minimum entropy procedure had higher precision than did both staircase procedures with a small step size for about $45 \mathrm{~s}$ right after the change in threshold. Moreover, the estimation precision of the minimum entropy procedure with PD \#3 was higher than the precision of the random staircase procedure after about $45 \mathrm{~s}$. The time necessary for the precision to return to baseline precision was about $50-70 \mathrm{~s}$ for the simple staircase and random staircase procedures and 100-120 s for the minimum entropy procedure.

\section{HUMAN SUBJECT STUDY}

In our human subject experiment, we chose to compare only the simple staircase and random staircase stimulus selection procedures. Logistic regression was used for threshold estimation in both cases.

\section{Method}

\section{Subjects}

The two stimulus selection procedures were compared in a psychophysical experiment with 31 pain-free human subjects ( 20 men and 11 women; 3 left-handed) 19-32 years of age (mean $=24.4$ and SD=2.9). The Medical Ethics Committee Twente approved all experimental procedures. All subjects provided written informed consent and were rewarded with a gift voucher after their participation in the experiment. 


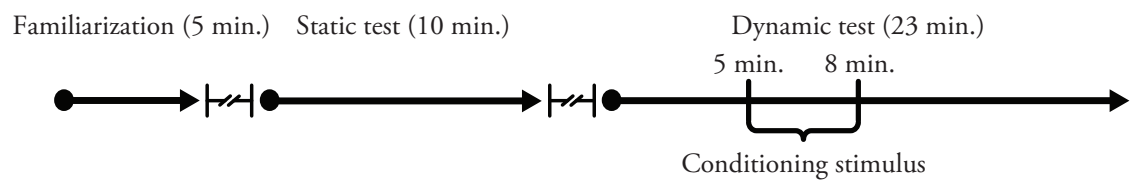

Figure 2-5 Schematic representation of the experiment time line.

\section{Test stimuli}

The test stimuli consisted of single cathodic square-wave electrical pulses with a pulse width of $525 \mu$ s. These stimuli were applied to subjects using a compound electrode that was attached to their left forearm and was connected to a stimulator (Steenbergen et al., 2012). The compound electrode consisted of an array of five needles and four flat electrodes (Figure 2-4). The needles served as the cathode, and the flat electrodes as anode. A needle electrode was used for electrocutaneous stimulation, since it has been shown to allow selective stimulation of nociceptive related $A \delta$-fibers when using current amplitudes below twice the perception threshold (Inui \& Kakigi, 2012; Mouraux, Iannetti, \& Plaghki, 2010).

The stimulator was developed by our group and is similar to the one used by Roosink, Buitenweg, Renzenbrink, Geurts, and IJzerman (2011), Steenbergen et al. (2012), and van der Heide, Buitenweg, Marani, and Rutten (2009). A custom computer program (written in LabVIEW 2011, SP1) controlled all stimulation procedures, as well as the registration of stimulus amplitudes in $\mathrm{mA}$, time in milliseconds, and responses to stimuli. Interstimulus interval times were randomly varied between 600 and 1,000 ms.

\section{Conditioning stimulus}

A 3-min cold pressor task was used as the nociceptive conditioning stimulus (Mitchell, MacDonald, \& Brodie, 2004; Pud et al., 2009; Talbot, Duncan, Bushnell, \& Boyer, 1987). Subjects were asked to immerse their right hand up to the wrist in a polystyrene container filled with water and crushed ice (water temperature was between $0^{\circ}$ and $3^{\circ}$ Celsius). Subjects were allowed to remove their hand from the water when the pain was no longer tolerable. However, they were instructed to continue with the protocol.

\section{Protocol}

The simple staircase procedure was compared with the random staircase procedure, both using logistic regression to estimate the thresholds. Momentary thresholds were estimated after every stimulus by using the 25 preceding stimulus-response pairs. 


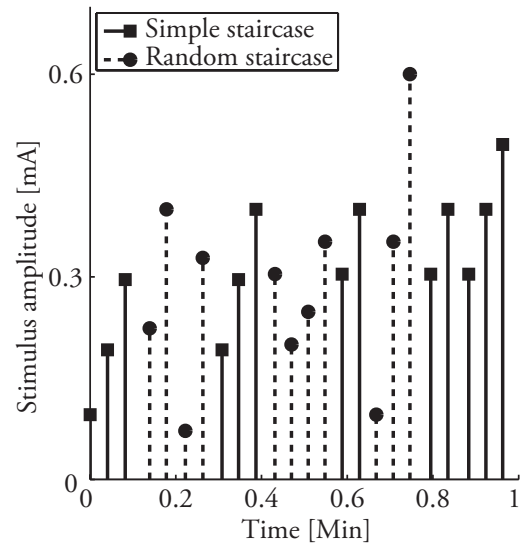

A

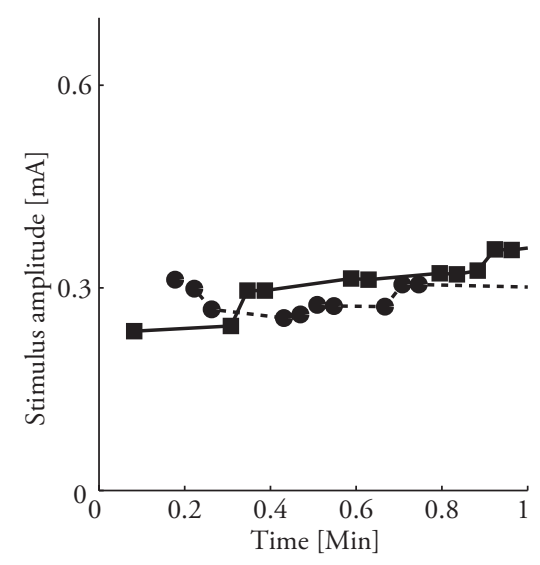

B

Figure 2-6 Sample section of stimulus application in the human subject study. Stimuli were randomly selected, but balanced, by either the simple staircase procedure (circles) or the random staircase procedure (squares). (A) Open marks indicate not perceived stimuli; filled markers indicate perceived stimuli. (B) Whenever a stimulus was perceived, a new threshold was estimated on the basis of the 25 preceding stimulus-response pairs.

In the simple staircase and random staircase procedures, a step size of $0.1 \mathrm{~mA}$ was used for both ascending and descending stairs. The random staircase procedure initially used a set of five amplitudes (i.e., NoA =5), equidistantly separated by between 0 and $0.3 \mathrm{~mA}$.

\section{Procedure}

The experiment procedure was divided into two tests: a 10-min static test followed by a 23-min dynamic test. The dynamic test included a cold pressor task between the $5^{\text {th }}$ and $8^{\text {th }}$ min (Figure 2-5). Subjects were instructed to indicate a perceived stimulus by releasing a response button and pressing the button again after about half a second to a second. After each perceived stimulus, the stimulus selection method was randomly selected to be either the simple or the random staircase procedure, with an equal number of trials for each method (Figure 2-6). Subjects were familiarized with the test stimuli before the start of each test by applying several test stimuli of various amplitudes.

\section{Data analysis}

All data preparation was performed in MATLAB 7.14 (MathWorks, Inc., Natick, MA). Linear mixed model analyses (LMMs) were performed in SPSS Statistics 20.0. The estimation method in the LMM was set to maximum likelihood, which was reported to be a better estimator of fixed effects (Twisk, 2006). Default settings were used for all other options in all LMMs unless stated otherwise. All other analyses were performed in 
MATLAB. Estimated thresholds during the first 2 min were excluded from the data set of each subject and each test to exclude possible procedural start-up effects.

Static test The LMM included intercept, time, procedure, and interaction between time and procedure as fixed effects and intercept, subjects, and time as random effects (covariance type: AR(1): Heterogeneous) to test whether the procedure and time had an effect on thresholds. Residuals of the model were saved and exported to MATLAB. We assumed that higher variance in the residuals indicated lower precision. A one-tailed two sample F-test was used to determine whether the variances of the residuals were unequal.

Dynamic test The LMM included intercept, time, procedure, conditioning stimulus, the interaction between time and procedure, and the interaction between conditioning stimulus and time as fixed effects to test whether procedure, time, and conditioning stimulus had an effect on thresholds. Intercepts, subjects, time, and conditioning stimulus were included as random effects (covariance type: AR(1): Heterogeneous). Since subjects were allowed to remove their hand from the water whenever they wanted, the conditioning stimulus time varied. For this reason, we partitioned the results of each test into three time intervals: before, during, and after the conditioning stimulus.

\section{Results}

A total of 31 subjects participated in the experiment. One subject was removed from the study after becoming unwell during the dynamic test, leaving a total subject population of $\mathrm{n}=30$.

\section{Static test}

Figure 2-7A presents the means and standard deviations of the threshold estimates over time for the static test. The standard deviations were due to a combination of intra- and intersubject effects and can therefore not be compared with the precisions presented in Figure 2-2. As can be seen from the graph in Figure 2-7A, the threshold gradually increased over time. In 3 subjects, the simple staircase procedure produced higher threshold estimates between 4.5 and 7 min than did the random staircase procedure. This was also briefly the case between 8 and $9 \mathrm{~min}$.

Table 2-2 presents the results of the LMM analysis. The thresholds significantly increased over time. No significant effect was found for procedure, nor was there a significant interaction effect between procedure and time. Table 2-3 presents the linear regression parameters corresponding to the significant effects. Moreover, a significant 


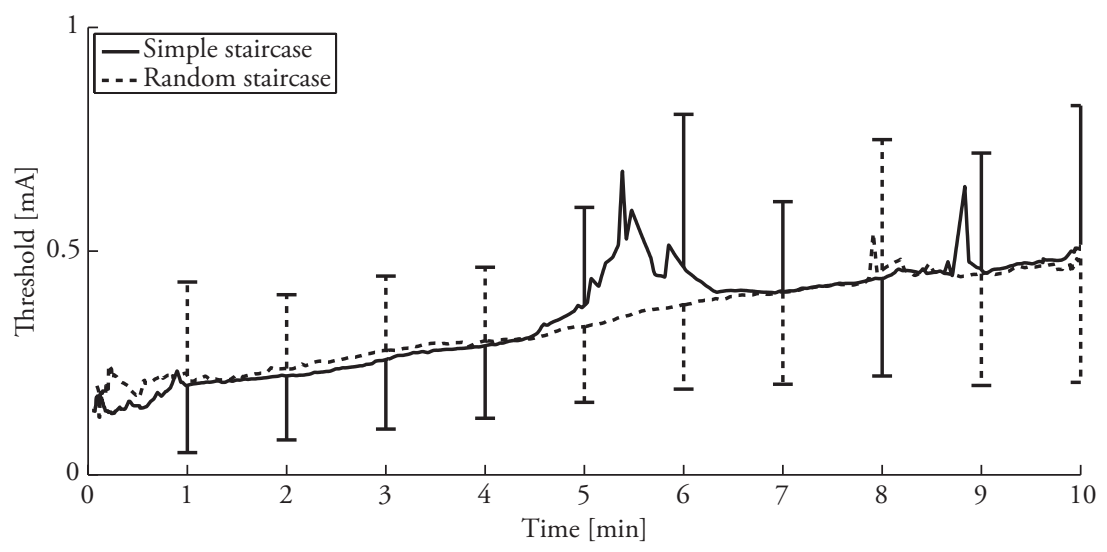

A

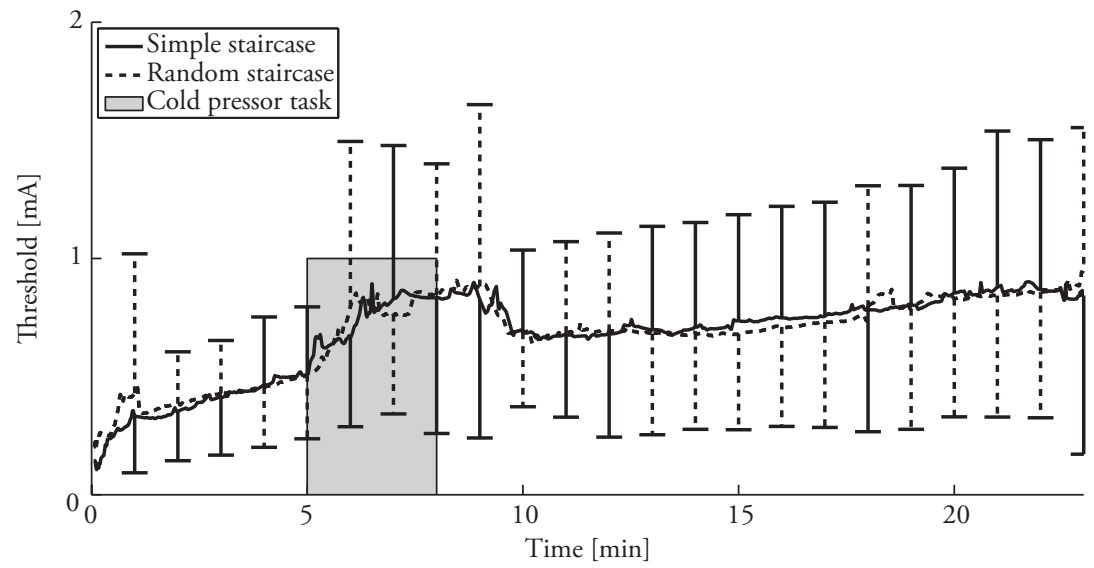

B

Figure 2-7 Human experiment results: mean and standard deviations of threshold estimates over time. (A) Estimated thresholds during the static test. (B) Estimated thresholds during the dynamic test. A conditioning stimulus is applied between the $5^{\text {th }}$ and $8^{\text {th }}$ min.

difference was found between the variances of the LMM output residuals, $\mathrm{F}(2522$, $2507)=8.44, p<.001$. Specifically, the variance of the residuals of the simple staircase procedure was found to be higher than that of the random staircase procedure.

Table 2-2 Linear mixed model results of fixed effects for the static test.

\begin{tabular}{llcr}
\hline Factor & $d f^{\text {a }}$ & \multicolumn{2}{l}{$F$} \\
\hline Procedure & $1 / 4942$ & 0.492 & .483 \\
Time & $1 / 30$ & 33.7 & $<.001$ \\
Procedure $\times$ Time & $1 / 4942$ & 2.19 & .139 \\
\hline
\end{tabular}

${ }^{a}$ Numerator/denominator degrees of freedom 
Table 2-3 Regression parameters of significant fixed effects and corresponding confidence intervals.

\begin{tabular}{llll}
\hline Parameter & Estimate & \multicolumn{2}{l}{$95 \%$ Confidence Interval } \\
\hline Intercept & $0.188[\mathrm{~mA}]$ & {$[0.127$} & $0.247]$ \\
Time & $0.0307[(\mathrm{~mA}) / \mathrm{min}]$ & {$[0.0191$} & $0.424]$ \\
\hline
\end{tabular}

\section{Dynamic test}

Twenty-four subjects immersed their hand in the water for the full $3 \mathrm{~min}$. The other 6 subjects immersed their hand for $88 \pm 34 \mathrm{~s}$ (mean \pm SD). Figure $2-7 \mathrm{~B}$ presents the means and standard deviations of the threshold estimates over time for the dynamic test. As can be seen from the two graphs, the estimated thresholds from the two procedures were similar, including the interval during the cold pressor task (between the $5^{\text {th }}$ and $8^{\text {th }} \mathrm{min}$ ). As in the static test, a gradual increase of threshold over time was visible.

It can be seen from Figure 2-7B that the thresholds increased immediately after the immersion of the hand into the water. Moreover, after removal of the hand, the estimated threshold did not immediately decrease but remained elevated for about 2 $\min$.

The results of the LMM analysis are presented in Table 2-4. Significant effects were found for conditioning stimulus, time, the interaction effect between procedure and time, and the interaction effect between conditioning stimulus and time. No significant effect was found for procedure. Corresponding linear regression parameters for the significant effects are presented in Table 2-5.

\section{DISCUSSION AND CONCLUSIONS}

We compared the performance of different stimulus selection procedures and threshold estimation methods for their use in threshold tracking experiments. The bias and precision of the threshold estimates (using either logistic regression or Bayesian estimation) of a simple staircase, a random staircase, and a minimum entropy procedure were compared

Table 2-4 Linear mixed model results of fixed effects for the dynamic test.

\begin{tabular}{lllr}
\hline Factor & $d f^{\mathrm{a}}$ & \multicolumn{1}{l}{$p$} & \multicolumn{1}{l}{$p$} \\
\hline Procedure & $1 / 13067$ & 1.43 & .232 \\
Conditioning stimulus & $2 / 30$ & 41.5 & $<.001$ \\
Time & $1 / 18$ & 62.0 & $<.001$ \\
Procedure $\times$ Time & $1 / 13054$ & 4.35 & .037 \\
Conditioning stimulus $\times$ Time & $2 / 31$ & 20.2 & $<.001$ \\
\hline
\end{tabular}

${ }^{\text {a} N u m e r a t o r / d e n o m i n a t o r ~ d e g r e e s ~ o f ~ f r e e d o m ~}$ 
by means of Monte Carlo simulations. Subsequently, we conducted a human subject study to compare the simple staircase and random staircase procedures.

\section{Monte Carlo simulations}

We performed two types of simulations: The first simulated a stationary threshold, and the second a non-stationary threshold. Various procedure-specific settings (e.g., of step size) were used in the simulations.

When the threshold was stationary, we observed a negligible bias in the threshold estimations in most cases. On the other hand, nonnegligible biases were observed when the threshold was not stationary (Figure 2-3). But, after the point of change in the threshold, the time to reach a negligible bias was similar in all simulations. Even though we did not vary the size of the moving window in the non-stationary threshold situation, we do expect the time required to return to a negligible bias to be dependent on the size of the moving window. Moreover, we expect that smaller and larger windows will result in a faster and slower return to negligible bias, respectively. However, smaller windows also reduced the estimation precision (Figure 2-2). Therefore, a trade-off between window size and estimation precision must be made. A larger window size is more appropriate when thresholds are stationary, while smaller window sizes are more appropriate when thresholds are expected to change.

When comparing the estimation precision between the different stimulus selection procedures, we observed that estimates made with the simple staircase procedure and minimum entropy procedure (with an appropriately chosen prior distribution) had a higher precision than those made with the random staircase procedure. In the stationary

Table 2-5 Regression parameters of significant fixed effects and corresponding confidence intervals.

\begin{tabular}{llll}
\hline Parameter & Estimate & \multicolumn{2}{l}{$95 \%$ Confidence Interval } \\
\hline Intercept & $0.628[\mathrm{~mA}]$ & {$[0.512$} & $0.764]$ \\
Conditioning stimulus & & & \\
$\quad$ Before cold pressor task & $-0.355[\mathrm{~mA}]$ & {$[-0.454$} & $-0.256]$ \\
$\quad$ During cold pressor task & $-0.418[\mathrm{~mA}]$ & {$[-0.604$} & $-0.232]$ \\
Time & $0.00797[(\mathrm{~mA}) / \mathrm{min}]$ & {$[-0.0004$} & $-0.0163]$ \\
Procedure $\times$ Time & & & \\
$\quad$ Simple staircase & $0.00111[(\mathrm{~mA}) / \mathrm{min}]$ & {$[0$} & $0.00216]$ \\
Conditioning stimulus $\times$ Time & & & \\
$\quad$ Before cold pressor task & $0.038[(\mathrm{~mA}) / \mathrm{min}]$ & {$[0.0231$} & $0.0529]$ \\
During cold pressor task & $0.0678[(\mathrm{~mA}) / \mathrm{min}]$ & {$[0.0155$} & $0.120]$ \\
\hline
\end{tabular}


threshold simulation, we found that the estimation precision of the minimum entropy procedure was more sensitive to different procedure-specific settings than was either of the staircase procedures. Moreover, the random staircase procedure was the least sensitive to different procedure settings. Likewise, in the non-stationary threshold simulations, the minimum entropy procedure showed higher sensitivity to the various settings and needed more time to converge to its baseline precision than did both the simple staircase procedure and random staircase procedure. However, it should be noted that the precision of the minimum entropy procedure was higher than the precision of the random staircase procedure after about $45 \mathrm{~s}$ after the change in threshold, but only with an appropriate prior distribution. On the other hand, small step sizes in the simple staircase procedure might result in a major loss in estimation precision. From this, we concluded that estimation precision can become very low if inappropriate procedure settings are chosen prior to a human subject experiment. Therefore, if little is known about the threshold and its behavior in a human subject experiment, we recommend using the random staircase procedure, since it provides more robust estimations than do the simple staircase and minimum entropy procedures. Otherwise, the simple staircase and minimum entropy procedures may be more appropriate, since their estimation precision (with an appropriate choice of settings) is higher than that of the random staircase procedure.

Regarding the estimation method, we observed that, in many cases, both logistic regression and Bayesian estimation resulted in a similar estimation precision (Figure 2-2). For Bayesian estimation, the precision was greatly reduced for both the simple staircase procedure and the minimum entropy procedure and, to a lesser extent, for the random staircase procedure, when the true slope parameter $\beta$ was near the upper limit of the PD (i.e., when PD \#1 and PD \#2 were used; Table 2-1). This implies that the precision of Bayesian estimation depends more on a priori choices than does that of logistic regression, which can be problematic when little information is available about the psychometric function parameters prior to a human subject experiment. Increasing the width of the probability distribution may solve this issue but will also increase the computational load (Kingdom \& Prins 2009, Kontsevich \& Tyler 1999, Kujala \& Lukka 2006). Using logistic regression as the estimation method may be a more appropriate solution to this issue, since it requires fewer a priori assumptions. However, one can always decide to use a Bayesian estimation method after the experiment. 


\section{Human subject study}

We compared the estimated thresholds of the simple staircase procedure with those of the random staircase procedure in a human subject study. For both procedures, logistic regression was used as the estimation method. Two tests were carried out in the human subject study: the first without a conditioning stimulus and the second with a 3-min cold pressor task as the conditioning stimulus. After each perceived stimulus during the experiment, the stimulus selection method was randomly selected to be either the simple or the random staircase procedure, with an equal number of trials for each method.

In humans, it is not possible to know the true threshold, and thus a bias cannot be determined. Moreover, it was difficult to calculate the precision in our human subject experiment because of inter-subject variability and possible habituation effects. However, the LMM used in this study modeled the random effects between subjects, as well as the effects of time. Therefore, higher variance in the LMM residuals implies a lower estimation precision, and vice versa.

We did not find differences in estimated thresholds between the simple staircase procedure and the random staircase procedure. In addition, no interaction effects between time and stimulus selection procedure were found, implying that the estimated thresholds were similar for the two procedures. We observed that the variance of the residuals of the fitted LMM was lower and, thus, the precision was higher for the random staircase procedure than for the simple staircase procedure. This observation was in contrast to the findings of the Monte Carlo simulations, which showed that in most cases, the estimations were more precise with the simple staircase than with the random staircase procedure. However, we also observed in the simulations that the precision of the simple staircase was more sensitive to various procedure settings than was that of the random staircase. Moreover, the higher thresholds estimated by the simple staircase between the $4^{\text {th }}$ and $7^{\text {th }}$ min in the static test could have caused higher variance in the residuals. Conversely, we found that this increase stemmed from 3 subjects only. Nevertheless, we ascribed the higher variance in the residuals to a lower estimation precision with the simple staircase procedure.

We found habituation effects over time in both the static and dynamic experiments. Thresholds also increased during the conditioning stimulus. As can be seen from Figure 2-7b, thresholds immediately increased after the start of the conditioning stimulus but required about 1-2 min before reaching the highest threshold. Van Wijk and Veldhuijzen (2010) reported that in most studies, the prolonged effect of the conditioning stimulus 
lasted less than $5 \mathrm{~min}$ and, in a few cases, up to $8 \mathrm{~min}$. We observed that the threshold started to decrease about $1 \mathrm{~min}$ after the conditioning stimulus was removed, although the conditioning stimulus effects were still visible up to about $2 \mathrm{~min}$ after stimulus removal. In the simulations, we observed that the threshold estimates immediately responded to a change in the true threshold, with no increasing delays. Therefore, we concluded that the conditioning stimulus caused the prolonged effect.

As is demonstrated by the presented data, we were able to track a nociceptive perception threshold over time. Since relatively low stimulus amplitudes were required, the perception of a stimulus might be sensitive to variations between subjects' levels of attention. Therefore, we were unable to conclude with certainty that the increased threshold during the conditioning stimulus was due to inhibitory effects on the nociceptive system. Moreover, the prolonged elevated threshold after applying the conditioning stimulus might be due to the fact that the hand needs more time to warm up after removing the conditioning stimulus than to cool down at the beginning of the conditioning stimulus. An experiment where subjects immerse their hands in a comfortably warm bath immediately after the conditioning stimulus may provide more insight into this phenomenon.

\section{Conclusion}

Monte Carlo simulations and a human subject study were used to demonstrate the possibility of tracking changes in threshold over time. We found that logistic regression and Bayesian estimation resulted in similar estimation precision only when the prior probability distributions were chosen appropriately. However, logistic regression estimations were more precise than Bayesian estimations when the slope parameter was near the edge of the prior probability distribution. Overall, the random staircase procedure exhibited the lowest sensitivity to the procedure settings. The minimum entropy procedure showed lower precision than did both the simple staircase and random staircase procedures when the slope parameter was near the edge of the prior probability distribution. The random staircase procedure was found to have higher estimation precision than the simple staircase procedure in the human subject experiments.

Therefore, we recommend the use of the random staircase over the simple staircase procedure, in combination with logistic regression, for non-stationary nociceptive threshold tracking experiments. 


\section{REFERENCES}

Cornsweet, T. N. (1962). The staircase-method in psychophysics. The American Journal of Psychology, 75(3), $485-491$.

Ehrenstein, W. H., \& Ehrenstein, A. (1999). Psychophysical methods. Modern Techniques in Neuroscience Research, 12111241.

Gescheider, G. A. (1985). Psychophysics: method, theory, and application: L. Erlbaum Associates.

Harvey, L. O. (1986). Efficient Estimation of Sensory Thresholds. Behavior Research Methods, Instruments, \& Computers, $18(6), 623-632$.

Hosmer, D. W., \& Lemeshow, S. (2000). Applied Logistic Regression: Wiley.

Inui, K., \& Kakigi, R. (2012). Pain perception in humans: use of intraepidermal electrical stimulation. Journal of Neurology, Neurosurgery, and Psychiatry, 83(5), 551-556.

King-Smith, P. E., \& Rose, D. (1997). Principles of an adaptive method for measuring the slope of the psychometric function. Vision Research, 37(12), 1595-1604.

Kingdom, F. A. A., \& Prins, N. (2009). Psychophysics: A Practical Introduction: Elsevier Science \& Technology.

Klein, S. A. (2001). Measuring, estimating, and understanding the psychometric function: a commentary. Perception $\&$ Psychophysics, 63(8), 1421-1455.

Kontsevich, L. L., \& Tyler, C. W. (1999). Bayesian adaptive estimation of psychometric slope and threshold. Vision Research, 39(16), 2729-2737.

Kujala, J. V., \& Lukka, T. J. (2006). Bayesian adaptive estimation: The next dimension. Journal of Mathematical Psychology, 50(4), 369-389.

Leek, M. R. (2001). Adaptive procedures in psychophysical research. Perception \& Psychophysics, 63(8), 1279-1292.

Levitt, H. (1971). Transformed up-down methods in psychoacoustics. Journal of the Acoustical Society of America, 49(2).

Mitchell, L. A., MacDonald, R. A., \& Brodie, E. E. (2004). Temperature and the cold pressor test. The Journal of Pain, 5(4), 233-237.

Mouraux, A., Iannetti, G. D., \& Plaghki, L. (2010). Low intensity intra-epidermal electrical stimulation can activate A $\delta$-nociceptors selectively. Pain, 150(1), 199-207.

Pud, D., Granovsky, Y., \& Yarnitsky, D. (2009). The methodology of experimentally induced diffuse noxious inhibitory control (DNIC)-like effect in humans. Pain, 144(1-2), 16-19.

Roosink, M., Buitenweg, J. R., Renzenbrink, G. J., Geurts, A. C., \& IJzerman, M. J. (2011). Altered cortical somatosensory processing in chronic stroke: A relationship with post-stroke shoulder pain. NeuroRehabilitation, 28(4), 331-344.

Simpson, W. A. (1988). The method of constant stimuli is efficient. Perception \& Psychophysics, 44(5), $433-436$.

Steenbergen, P., Buitenweg, J. R., Trojan, J., van der Heide, E. M., van den Heuvel, T., Flor, H., \& Veltink, P. H. (2012). A system for inducing concurrent tactile and nociceptive sensations at the same site using electrocutaneous stimulation. Behavior Research Methods, 44(4), 924-933

Talbot, J. D., Duncan, G. H., Bushnell, M. C., \& Boyer, M. (1987). Diffuse noxious inhibitory controls (DNICs): psychophysical evidence in man for intersegmental suppression of noxious heat perception by cold pressor pain. Pain, 30(2), 221-232.

Treutwein, B. (1995). Adaptive psychophysical procedures. Vision Research, 35(17), 2503-2522.

Treutwein, B. (1997). YAAP: yet another adaptive procedure. Spatial Vision, 11(1), 129-134.

Treutwein, B., Rentschler, I., \& Caelli, T. (1989). Perceptual spatial frequency-orientation surface: psychophysics and line element theory. Biological Cybernetics, 60(4), 285-295.

Treutwein, B., \& Strasburger, H. (1999). Fitting the psychometric function. Perception \& Psychophysics, 61(1), 87-106.

Twisk, J. W. R. (2006). Applied Multilevel Analysis: A Practical Guide for Medical Researchers: Cambridge University Press.

van der Heide, E. M., Buitenweg, J. R., Marani, E., \& Rutten, W. L. (2009). Single pulse and pulse train modulation of cutaneous electrical stimulation: a comparison of methods. Journal of Clinical Neurophysiology, 26(1), 54-60.

Van Wijk, G., \& Veldhuijzen, D. S. (2010). Perspective on diffuse noxious inhibitory controls as a model of endogenous pain modulation in clinical pain syndromes. The Journal of Pain, 11(5), 408-419. 



\section{Chapter 3}

Observation of time-dependent psychophysical functions and accounting for threshold drifts

Robert J. Doll, Peter H. Veltink, Jan R. Buitenweg

Attention, Perception, \& Psychophysics (2015) $77(4), 1440-1447$

DOI $10.3758 / s 13414-015-0865-x$ 
Abstract Methods to obtain estimates of psychophysical functions are used in numerous fields, such as audiology, vision, and pain. Neurophysiological and psychological processes underlying this function are assumed to remain stationary throughout a psychophysical experiment. However, violation of this assumption (e.g., due to habituation or changing decisional factors) likely affects the estimates of psychophysical parameters. We used computer simulations to study how non-stationary processes, resulting in a timedependent psychophysical function, affect threshold and slope estimates. Moreover, we propose methods to improve the estimation quality when stationarity is violated. A psychophysical detection experiment was modeled as a stochastic process ruled by a logistic psychophysical function. The threshold was modeled to drift over time and was defined as either a linear or nonlinear function. Threshold and slope estimates were obtained by using three estimation procedures: a static procedure assuming stationarity, a relaxed procedure accounting for linear effects of time, and a threshold tracking paradigm. For illustrative purposes, data acquired from two human subjects were used to estimate their thresholds and slopes using all estimation procedures. Threshold estimates obtained by all estimations procedures were similar to the mean true threshold. However, due to threshold drift, the slope was underestimated by the static procedure. The relaxed procedure only underestimated the slope when the threshold drifted nonlinearly over time. The tracking paradigm performed best and therefore, we recommend using the tracking paradigm in human psychophysical detection experiments to obtain estimates of the threshold and slope and to identify the mode of non-stationarity. 


\section{INTRODUCTION}

Methods to obtain estimates of psychophysical functions describing processes of underlying sensory mechanisms are used in many fields of study (e.g., hearing, vision, or tactile studies). The psychophysical function describes the probability that a stimulus is detected given the stimulus strength and has a sigmoidal form (Gold \& Ding, 2013; Leek, Hanna, \& Marshall, 1991). Psychophysical parameters describing the psychophysical function, such as the threshold and the slope, can serve as a quantifier of disease, as in studies of audiology (McFadden, 1983), vision (Chauhan, Tompkins, LeBlanc, \& McCormick, 1993; Wallis, Baker, Meese, \& Georgeson, 2013), and pain (Sandkühler, 2009).

In a simple stimulus detection experiment, subjects are presented several stimuli with varying amplitudes to which the corresponding responses (i.e., detected or not detected) are recorded. From these stimulus-response pairs (SRPs), an estimate of the psychophysical function can be obtained (see Kingdom and Prins (2009) for an introduction to psychophysical methods). The threshold parameter is a commonly used measure to describe this function and is defined as the amplitude resulting in a 50\% detection probability (Klein, 2001; Treutwein, 1995). The steepness of the psychophysical function at the threshold level is described by the slope parameter and provides information about the reliability of stimulus detection by the subject (Gold \& Ding, 2013; Strasburger, 2001).

In practice, the neurological and psychological processes underlying the psychophysical function are assumed to remain stationary throughout estimations of threshold and slope in psychophysical experiments. However, fatigue, loss of attention, or a change in decisional criteria (Fründ, Haenel, \& Wichmann, 2011; Leek et al., 1991) can result in violation of this assumption. As a result, estimates of the psychophysical threshold and slope might be impaired and are possibly unreliable.

A conceivable consequence of violation of the stationarity assumption is demonstrated in Figure 3-1. Figure 3-1A presents how a threshold can drift within an experiment from time-point $\mathrm{A}$ to $\mathrm{B}$, resulting in a rightward shift of the psychophysical function on the stimulus axis. This rightward shift is depicted in Figure 3-1B. The two dashed lines depict the true psychophysical function at timepoints A and B, respectively. In between the two time-points, the function shifts as a linear function from time-point $A$ to $B$ while the slope parameter remains stationary. The solid black line represents the estimate of the psychophysical function from stimulus response pairs obtained between time-points 


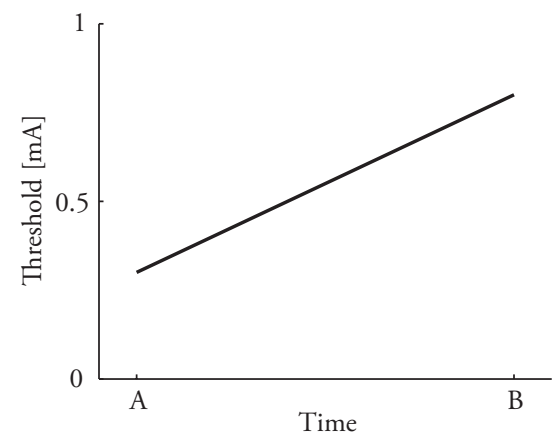

A

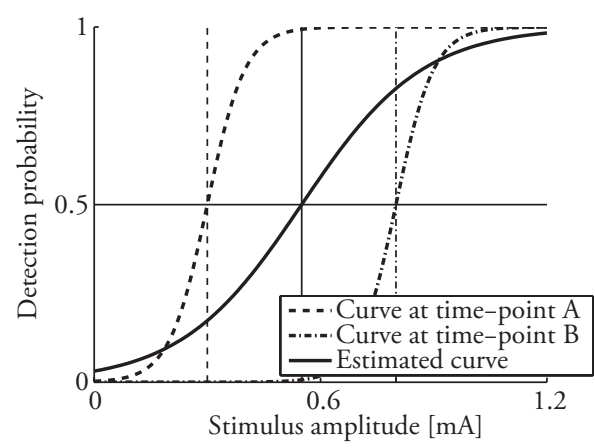

B

Figure 3-1 (A) True thresholds can drift over time resulting in a different threshold value at time-point B than at timepoint A. (B) A drift over time in the threshold can be represented as a shift of the psychophysical function to the right (e.g., from the dash-dotted curve at time-point A to the dashed curve at time-point B). A curve similar to the solid line will be estimated when stationarity of the threshold is assumed.

$\mathrm{A}$ and $\mathrm{B}$ when stationarity would have been assumed. As all stimulus response pairs contribute equally to the estimation, the estimate of the threshold will be similar to the averaged true threshold over the time interval A-B. As a result, the slope of the estimated curve is underestimated and therefore falsely suggests a lower accuracy of the subject to discriminate between stimulus intensities.

A practical example of where non-stationarities are known to occur is when estimating the nociceptive function. Changes in nociceptive thresholds can occur, for example, due to clinical interventions (Olesen et al., 2013) and experimental conditioning stimuli (Pud, Granovsky, \& Yarnitsky, 2009). Moreover, nociceptive detection thresholds can show a continuous increase over time during a 10-minute experiment (Chapter 2). Additionally, observation of the effect of time on estimations could be a relevant indicator of disease (e.g., reduced habituation is present in fibromyalgia and migraine patients (Smith et al., 2008; Valeriani et al., 2003)).

If unwanted, the effect of time on the psychophysical function can be minimized by carefully preparing the experiment, such as increasing the time between two consecutive stimuli (von Dincklage, Olbrich, Baars, \& Rehberg, 2013). However, these preparations could be impractical for clinical purposes, where only a limited amount of time is available for psychophysical recordings. For these purposes, it is important to be aware of the possibility that the stationarity assumption can be violated. Therefore, the purpose of this study was to observe how a non-stationary process, resulting in a timedependent psychophysical function, can be identified and how this affects threshold and slope estimates. Moreover, we study how these estimates can be improved by two 
different estimation procedures.

As a first strategy to improve estimations of the psychophysical functions, the assumption of a stationary threshold could be relaxed by allowing time to have a linear effect on the threshold. Allowing this can be done by including the times at which stimuli were presented in the estimation process (e.g., include stimulation time as a covariate in (generalized) linear regression models). Doing so might improve the slope estimates. However, this improvement might depend on the type of non-stationarity of the underlying processes. Not only linear drifts of the psychophysical function but also more complex nonlinear drifts might occur (Milne, Kay, \& Irwin, 1991). As a second strategy, nonlinear changes in a threshold over time can be observed by using a threshold tracking paradigm. This paradigm uses a time-window including a number of the most recent SRPs to obtain a momentary estimate of the threshold (Chapter 2; von Dincklage, Hackbarth, Schneider, Baars, \& Rehberg, 2009). This window is shifted each time a new single SRP is obtained. Therefore, this paradigm could be used as a first identifier of non-stationary behavior.

How threshold and slope estimates are affected by non-stationary processes depends on its time-dependent properties and on the estimation procedure. In human psychophysical experiments, the true threshold and slope are unknown and thus can only be estimated. However, computer simulations allow the true psychophysical parameters to be defined and therefore allow evaluating the estimates. We used a Monte Carlo simulation approach to generate stimulus response pairs. A psychophysical function underlying a non-stationary process was modeled by means of a drifting threshold. The true threshold was defined as (1) a constant function, (2) a linear increasing function, or (3) a saturating exponential function. Three different estimation procedures were used to obtain estimates of the threshold and the slope of the psychophysical function using the simulated SRPs. In addition to the simulations, we illustrate the estimation procedures on data of two human subjects coming from a previous study (Chapter 2).

\section{METHODS}

Estimates of the psychophysical functions, in terms of thresholds and slopes coming from three different estimation procedures, were compared by means of Monte Carlo simulations. The simulation procedures described below are similar to the procedures described in Chapter 2. Several realizations of a psychophysical experiment were simulated using a stochastic psychophysical function to simulate the responses to stimulus amplitudes. Threshold drift was included in the simulations to model a non-stationary 
underlying process. The true threshold was defined as (1) a constant function, (2) a linear increasing function, and (3) a saturating exponential function with two different time constants. Thresholds and slopes were estimated using one out of the three estimation procedures described in the section "Threshold and slope estimation" below. For each of the 12 experiments presented in this paper, 10,000 realizations were simulated. In addition to the simulations, psychophysical data coming from two human subjects were used to illustrate the different estimation procedures in practice. All simulations and analysis were performed with MATLAB 8.1. (MathWorks, Inc., Natick, MA).

\section{Psychophysical model and model parameters}

The probability $p$ of detecting a stimulus of amplitude $x[\mathrm{~mA}]$ was modeled with a logistic psychophysical function:

$$
p(x ; \alpha(t), \beta)=(1+\exp (\beta(\alpha(t)-x)))^{-1},
$$

where $\alpha(t)$ and $\beta$ were the threshold and slope parameter of the psychophysical function, respectively. Threshold drift over time was modeled as either a linear function $\alpha(t)=\alpha_{0}+\theta t$, with $\theta$ set to either 0 or $0.1[(\mathrm{~mA}) / \mathrm{min}]$, or as a saturating exponential function: $\alpha(t)=\alpha_{0}+\left(1-\exp \left(-\frac{t}{\tau}\right)\right)$, with the time-constant $\tau$ set to 1 or $3[\mathrm{~min}]$. The initial threshold $\alpha_{0}$ and slope $\beta$ were set to $0.3[\mathrm{~mA}]$ and $20[1 /(\mathrm{mA})]$, respectively. All simulated thresholds over time are presented in Figure 3-2A.

Simulated responses to a given stimulus were classified as detected if $p(x)>\varepsilon$, where $\varepsilon$ is a random number drawn from a uniform distribution between 0 and 1 , and as not detected otherwise. The random number generator was shuffled each time a new simulation was started to ensure uncorrelated realizations of simulated responses.

\section{Stimulus selection}

For probing the modeled psychophysical functions by SRPs, stimulus amplitudes, $x$, were selected according to an adaptive probing procedure 1 described in Chapter 2. The procedure started with a predefined set of 5 equidistant stimulus amplitudes between 0 and $0.3 \mathrm{~mA}$, from which new stimulus amplitudes were randomly selected. All amplitudes in the set were increased by a step size of $0.1 \mathrm{~mA}$ after a not-detected stimulus and decreased with $0.1 \mathrm{~mA}$ after a detected stimulus (Figure 3-2B). 


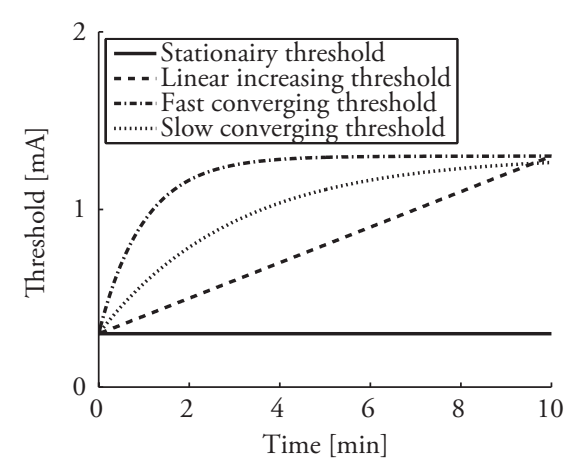

A

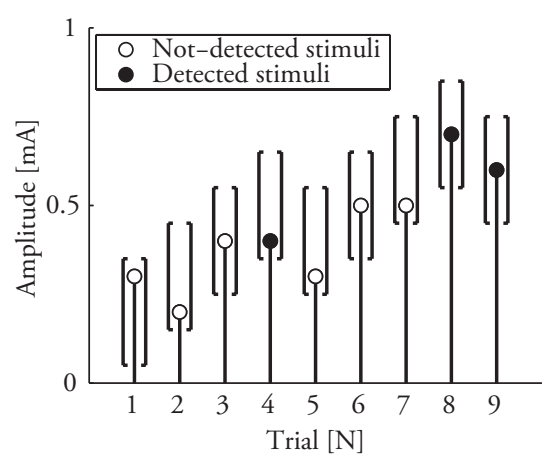

B

Figure 3-2 (A) Four thresholds functions were used: a constant threshold of 0.3 [mA], a linear drifting threshold with a drifting rate $0.1[(\mathrm{~mA}) / \mathrm{min}]$, a slow converging threshold with a time-constant of 3 [min], and a fast converging threshold with a time-constant of 1 [min]. (B) A typical example of the stimulus selection procedure used in the simulations. The brackets represent the set within which a stimulus can be randomly chosen. A not-detected stimulus results in an increase of the set while a detected stimulus results in a decrease of the set.

In our experimental paradigm, the time between two consecutive stimuli depends on the corresponding response to the previous stimulus. Human subjects hold a response button in order for the equipment to start applying electrical stimuli. If the button is not released after a stimulus is applied, a not detected stimulus is assumed. After a stimulus is detected, subjects are to release the button, and repress it again after about a second. This procedure results in a shorter inter-stimulus interval after a not detected stimulus than after a detected stimulus. In the simulations described in this study, inter-stimulus intervals were set to 1.5 and 3.5 seconds after a not-detected stimulus and a detected stimulus, respectively.

\section{Threshold and slope estimation}

Three estimation procedures were used to obtain threshold and slope estimates. The first and second estimation procedures were generalized linear regression models with a logit link function. The first procedure assumed a stationary threshold and slope throughout the experiments, whereas the second procedure assumed that time can have a linear effect on the detection probability. From here on, we refer to the first and second procedures as the static and relaxed procedures, respectively.

The static procedure was of the form:

$$
\operatorname{logit}(\pi)=b_{0}+b_{1} X
$$

where $\pi$ is the estimated detection probability, $b_{0}$ the intercept parameter and $b_{1}$ the 
slope parameter for the stimulus amplitude $X$ (i.e. $x$ in Equation (3-1)). The threshold was defined as the amplitude resulting in a 0.5 detection probability:

$$
\alpha=X(\pi=.5)=-\left(\frac{b_{0}}{b_{1}}\right) .
$$

The relaxed procedure was of the form:

$$
\operatorname{logit}(\pi)=b_{0}+b_{1} X+b_{2} T \text {, }
$$

where $b_{2}$ is the slope parameter for the time $T$ (i.e. $t$ in Equation (3-1)). The threshold $\alpha(t)$ was calculated by:

$$
\alpha(t)=X(t \mid \pi=.5)=-\left(\frac{b_{0}+t b_{2}}{b_{1}}\right) .
$$

Single threshold and slope estimates for the static and relaxed procedure were obtained by including all the available SRPs in each realization. The threshold estimate for the relaxed procedure were based on the detection probability at $t=5$ minutes.

For threshold tracks, a shifting time window was used to obtain momentary estimates of the threshold and the slope using the regression model used for the relaxed procedure (i.e., Equation (3-5) with $t=$ the time at the center of the window). The window included 25 SRPs: 12 preceding SRPs, 12 upcoming SRPs, and the current SRP. This resulted in several threshold and slope estimates per realizations. Therefore, to obtain a single estimate, the momentary estimates were averaged resulting in a single threshold and slope estimate per realization.

\section{Analysis}

Threshold and slope estimates were only included for analysis when the threshold estimate was between 0 and $5 \mathrm{~mA}$ and when the slope estimate was smaller than $50[1 /(\mathrm{mA})]$. All other estimates were considered unrealistic and therefore were excluded from the dataset. Slope estimates were found to be skewed and were therefore natural log-transformed. To produce equally spaced estimates, threshold and slope tracks were linearly interpolated using a rate of $1 \mathrm{~Hz}$ to prevent under sampling. 


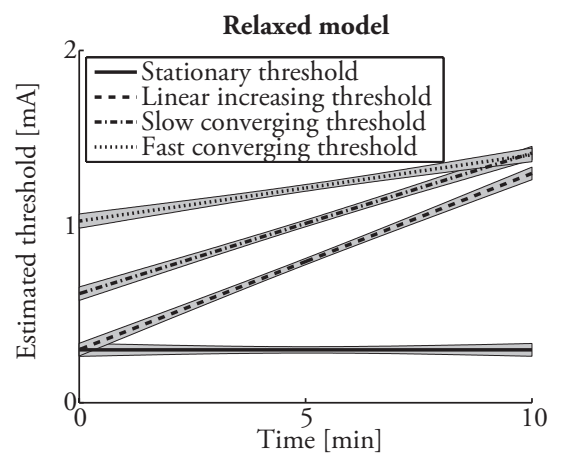

A

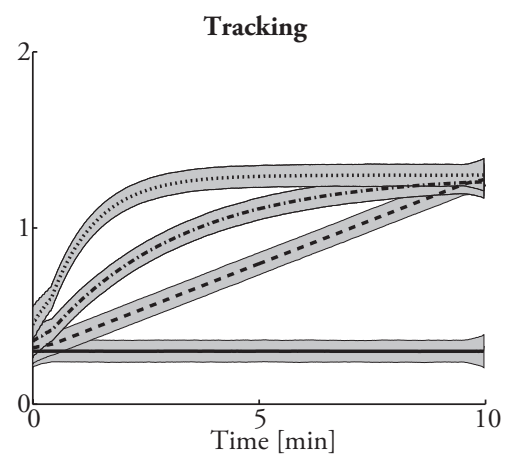

B

Figure 3-3 Mean and 95\% confidence intervals of time-dependent estimated thresholds (i.e., for the relaxed (A) and tracking (B) procedures).

\section{Human subject experiment}

Recordings of two human subjects who show a non-stationary threshold were selected from a previous study Chapter 2. These were used to illustrate the different estimation procedures described earlier. Single cathodic square-wave electrical stimuli (with a pulse width of $525 \mu \mathrm{s}$ ) using a 5 needle electrode (Steenbergen et al., 2012) were presented to the subjects' left forearm for 10 minutes. Single threshold estimates were obtained using both the static and relaxed procedures. Moreover, the threshold was tracked over time using the 25 most recent SRPs in the moving time window to obtain a momentary threshold. The relaxed procedure was used for threshold and slope estimation.

\section{RESULTS \\ Simulations}

The mean and $95 \%$ confidence intervals of threshold estimates over time obtained by the relaxed procedure and the tracking procedure are presented in Figure 3-3. The estimates are clearly dependent on time. The relaxed procedure approximates the nonlinear drift in the true threshold as linear drift, and, therefore results in estimations over time that do not follow the exponential form of the slow and fast converging thresholds. The tracking procedure, however, does follow the drift in threshold, regardless of the modeled drift.

Figure 3-4A presents the mean true threshold and the mean and 95\% confidence intervals of single threshold estimates obtained by all estimation procedures and all simulated psychophysical functions. Larger threshold estimates were obtained when the true threshold was increasing over time than when the threshold was kept constant. However, the estimates were similar to the mean true threshold for all estimation procedures. 


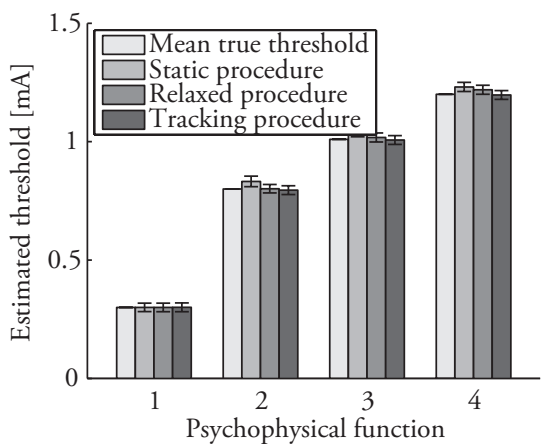

A

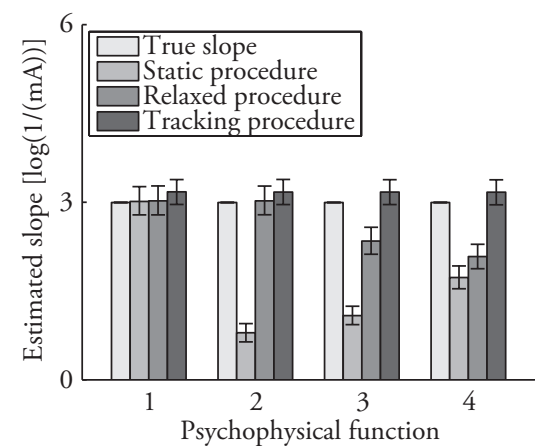

B

Figure 3-4 Mean and 95\% confidence intervals of estimated thresholds (A) and natural log-transformed slopes (B) for the different psychophysical functions ( 1 indicates the constant threshold, 2 the linear increasing threshold, 3 the slow converging threshold, and 4 the fast converging threshold.

Figure $3-4 \mathrm{~B}$ presents the mean and $95 \%$ confidence intervals of single log-transformed slope estimates obtained by all estimation procedures and all simulated psychophysical functions. When the true psychophysical function remained stationary, slope estimates were similar to the true slope. When the static procedure was used to estimate slopes of non-stationary psychophysical functions, slopes were underestimated, resulting in more gradual functions than the true function. Slopes were underestimated only when the true threshold was described as a saturated exponential function and estimated using the relaxed procedure. Slope estimates remained similar to the log transformed true slope value when using the tracking method, regardless of the simulated psychophysical function.

\section{Human subject experiment}

Figure 3-5 presents the data of two human subjects where the stimulus response pairs and corresponding threshold estimates are plotted. For subject A, a threshold estimate of $0.56 \mathrm{~mA}$ was obtained when the static procedure was used. Using the relaxed procedure resulted in threshold estimates ranging between 0.09 and $0.95 \mathrm{~mA}$ from the start to the end of the experiment. Thresholds estimated by the tracking procedure resulted in similar estimates as the relaxed procedure. Estimating a single threshold for subject $\mathrm{B}$, using the static procedure, resulted in an estimate of $0.31 \mathrm{~mA}$. Using the relaxed procedure resulted in an estimate starting at $0.18 \mathrm{~mA}$ and ending at $0.44 \mathrm{~mA}$ at the end of the experiment. The threshold track estimated that the threshold increased from 0.18 to $0.45 \mathrm{~mA}$ from the start until approximately 5 minutes of the experiment. It then gradually decreased to $0.25 \mathrm{~mA}$ until 8 minutes and then continued to increase again to approximately $0.44 \mathrm{~mA}$. 


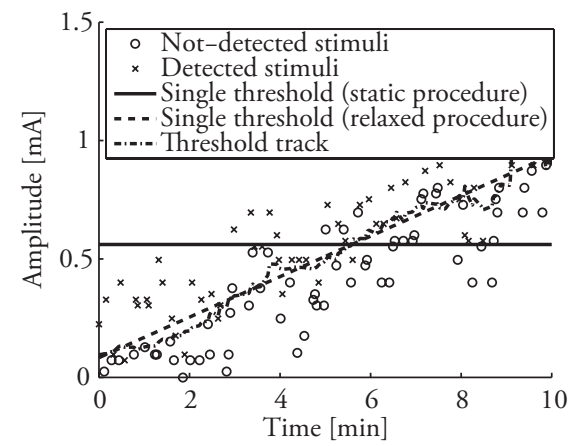

A

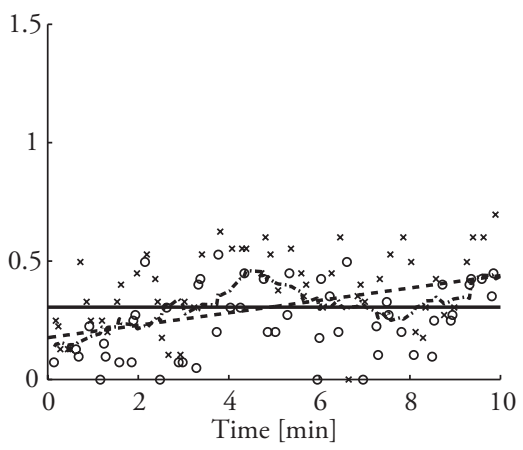

B

Figure 3-5 Human subject data of two subjects. Both graphs present stimuli and corresponding responses, where the crosses and circles indicate detected and not-detected stimuli, respectively. The solid black and dotted line represent a single estimated threshold using the static procedure (Equation (3-2)) and the relaxed procedures (Equation (3-4)), respectively. The dashed line represent the threshold track. The subject in (A) shows an increase in threshold over time, which could be assumed to be linear. However, the subject in (B) shows an effect of time on threshold where the linearity is debatable.

The psychophysical functions, obtained by estimating a single threshold and slope for each of the estimation procedures and each subject, are shown in Figure 3-6. Estimated thresholds are similar for the three estimation procedures. However, estimates of the slope are not similar. The slope for subject A was estimated to be $0.52,1.96$, or 2.78 $[\log (1 /(\mathrm{mA}))]$ by using the static, relaxed, or tracking procedure, respectively. The slope for subject $B$ was estimated to be $1.61,1.84$, or $2.56[\log (1 /(\mathrm{mA}))]$ by using the static, relaxed, or tracking procedure, respectively.

\section{DISCUSSION}

We used a Monte Carlo simulation approach to study the effect of non-stationary processes underlying the psychophysical function on the estimation of psychophysical thresholds and slopes. While the slope of the true psychophysical function was kept constant, the threshold was modeled as function of time. Estimates of the psychophysical threshold and slope were obtained from simulated stimulus response pairs, using three estimation procedures: static procedure, relaxed procedure, and threshold tracking procedure. In addition, we demonstrated the estimation procedures in two healthy human subjects for illustrative purposes.

When the processes underlying the psychophysical function resulted in a constant true threshold, estimates of the threshold obtained by the three estimation procedures were similar to the true threshold. Moreover, when the stationarity assumption was violated due to a drifting threshold, threshold estimates were similar to the mean 

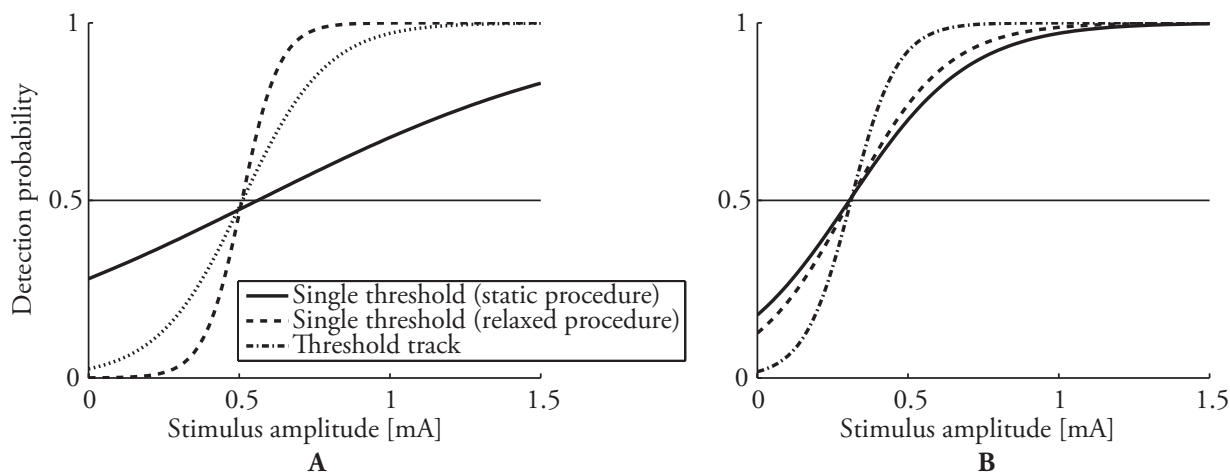

B

Figure 3-6 Estimated psychophysical functions using the different estimation procedures for the two human subjects.

true threshold value for all estimation procedures. However, it should be noted that approximately 240 SRPs were used for threshold estimation in this experiment. Given this number of SRPs, a high estimation precision is expected, which might be the reason why no relevant difference between the estimation procedures was found. Generally, the reliability of threshold estimates increases when more SRPs are included in the estimation process (Taylor, 1971). When fewer SRPs are available (e.g., fewer than 50), a difference between the results coming from the different estimation procedures might exist. Therefore, all three estimation procedures can be used to obtain threshold estimates whenever the threshold is the only parameter of interest in an experiment and a large number of SRPs are available.

When not only a threshold estimate is of interest, but also the reliability of stimulus detection by the subject, an estimate of the slope can be obtained as well. From the simulation results, it was found that slope estimates were similar whenever the threshold was constant over time. However, the static procedure returned biased slope estimates when the threshold was drifting over time. As mentioned in the introduction, the underestimation is due to the averaging of the psychophysical functions over time (Figure 3-1). Accounting for a linear effect of time on the threshold by using the relaxed procedure improved the slope estimates (Figure 3-4). However, even though estimates obtained by the relaxed procedure were better than the static procedure, they were still biased whenever the threshold was modeled as a nonlinear function. This bias was no longer visible when the tracking paradigm was used to obtain threshold and slope estimates. As the slope estimates obtained by the tracking procedure are similar or better than those obtained by the other two procedures, we recommend preferring the tracking procedure over the other procedures in experiments where estimates of the slope are required. 
In addition to threshold and slope estimates, an estimate of the effect of time on the threshold can be obtained by the relaxed procedure and tracking procedure. The relaxed procedure assumes that the threshold drifts over time with a linear rate. Nonlinear threshold changes are approximated as linear function, resulting in estimation biases. This is not the case when the tracking procedure was used; the tracking procedure assumes piecewise linearity of the threshold resulting in a lower bias, even when the threshold drifts with a nonlinear rate. However, as shown in Figure 3-3, the estimation confidence intervals are smallest when the relaxed procedure was used. This implies that the threshold estimation precision per time-point is higher when the relaxed procedure was used than when the tracking procedure was used. Therefore, if it is reasonable to state that the underlying process is either stationary or results in a linear drifting threshold, the relaxed procedure performs better than the tracking procedure. The tracking procedure could be used to obtain an indication on whether linearity could be assumed. For example, from the data obtained for subject A (Figure 3-5A and Figure 3-6A), it becomes immediately clear that the psychophysical curve obtained by the static procedure results in a poor fit but seems to be fit better by both the relaxed and tracking procedures. For subject $\mathrm{B}$, however, it is not immediately clear what model to fit to the data. The threshold track shows a non-monotonously drifting threshold, suggesting that neither the static nor the relaxed procedure is appropriate. Therefore, in human subject experiments, the tracking procedure can be used as an indication for threshold behavior over time. If linearity seems reasonable, the relaxed procedure can be used to obtain precise threshold estimates, otherwise, the tracking procedure is recommended.

In this study, a threshold was modeled as either constant or monotonous increasing. However, in practice, non-monotonous varying thresholds may occur as well. For example, in pain experiments, a conditioning stimulus, such as immersing an extremity into painful cold water, might induce a temporary change in the threshold (Pud et al., 2009). Estimating a single threshold in these cases does not reflect any dynamic properties of the underlying processes. Therefore, a time-profile of the threshold could then be obtained by either estimating several single thresholds during the experiment or by tracking the threshold using the tracking paradigm.

Another limitation of the present study was that the slope of the psychophysical function was defined to have a constant value. In human subject experiments, however, the slope might show changes over time as well. Assuming a stationary slope, while the slope is non-stationary is likely to affect the estimation process. However, because we 
did not model a time-dependent slope, further studies are necessary to identify its effect on the estimation quality.

\section{Conclusions}

We demonstrated that non-stationarities in processes underlying the psychophysical function resulting in threshold drift affect the estimation of thresholds and slopes. Slopes were underestimated, resulting in more gradual psychophysical functions than the true one. Accounting for linear effects of time on the threshold in the estimation process improves the slope estimates. However, slopes are still underestimated when the threshold drifts in a way that is not accounted for in the estimation model (i.e., nonlinear drift). Tracking the psychophysical function over time using a window shifting over time, and then averaging all estimates to obtain a single estimate, results in better threshold and slope estimates, regardless of the non-stationarity. We recommend using the tracking procedure in human psychophysical detection experiments to obtain estimates of the threshold and slope and to identify the mode of non-stationarity.

\section{REFERENCES}

Chauhan, B. C., Tompkins, J. D., LeBlanc, R. P., \& McCormick, T. A. (1993). Characteristics of frequency-of-seeing curves in normal subjects, patients with suspected glaucoma, and patients with glaucoma. Investigative Ophthalmology and Visual Science, 34(13), 3534-3540.

Cornsweet, T. N. (1962). The staircase-method in psychophysics. The American Journal of Psychology, 75(3), 485-491.

Fründ, I., Haenel, N. V., \& Wichmann, F. A. (2011). Inference for psychometric functions in the presence of nonstationary behavior. Journal of Vision, 11(6), 1-19.

Gold, J. I., \& Ding, L. (2013). How mechanisms of perceptual decisionmaking affect the psychometric function. Progress in Neurobiology, 103, 98-114.

Kingdom, F. A. A., \& Prins, N. (2009). Psychophysics: A Practical Introduction. London: Academic Press.

Klein, S. A. (2001). Measuring, estimating, and understanding the psychometric function: A commentary. Perception and Psychophysics, 63(8), 1421-1455.

Leek, M. R., Hanna, T. E., \& Marshall, L. (1991). An interleaved tracking procedure to monitor unstable psychometric functions. Journal of the Acoustical Society of America, 90(3), 1385-1397.

McFadden, D. F. L. (1983). Audition: Some relations between normal and pathalogical hearing. Annual Review of Psychology, 34(1), 95.

Milne, R. J., Kay, N. E., \& Irwin, R. J. (1991). Habituation to repeated painful and non-painful cutaneous stimuli: A quantitative psychophysical study. Experimental Brain Research, 87(2), 438-444.

Olesen, S. S., Graversen, C., Bouwense, S. A. W., van Goor, H., WilderSmith, O. H. G., \& Drewes, A. M. (2013). Quantitative Sensory Testing Predicts Pregabalin Efficacy in Painful Chronic Pancreatitis. PLoS One, 8(3).

Pud, D., Granovsky, Y., \& Yarnitsky, D. (2009). The methodology of experimentally induced diffuse noxious inhibitory control (DNIC)like effect in humans. Pain, 144(1-2), 16-19.

Sandkühler, J. (2009). Models and mechanisms of hyperalgesia and allodynia. Physiological Reviews, 89(2), $707-758$.

Smith, B. W., Tooley, E. M., Montague, E. Q., Robinson, A. E., Cosper, C. J., \& Mullins, P. G. (2008). Habituation and sensitization to heat and cold pain in women with fibromyalgia and healthy controls. Pain, 140(3), 420-428 
Steenbergen, P., Buitenweg, J. R., Trojan, J., van der Heide, E. M., van den Heuvel, T., Flor, H., \& Veltink, P. H. (2012). A system for inducing concurrent tactile and nociceptive sensations at the same site using electrocutaneous stimulation. Behavior Research Methods, 44(4), 924-933.

Strasburger, H. (2001). Converting between measures of slope of the psychometric function. Perception and Psychophysics, 63(8), 1348-1355.

Taylor, M. M. (1971). On the efficiency of psychophysical measurement. Journal of the Acoustical Society of America, 49(2), Suppl 2:505-508.

Treutwein, B. (1995). Adaptive psychophysical procedures. Vision Research, 35(17), 2503-2522.

Valeriani, M., de Tommaso, M., Restuccia, D., Le Pera, D., Guido, M., Iannetti, G. D., \& Cruccu, G. (2003). Reduced habituation to experimental pain in migraine patients: A CO2 laser evoked potential study. Pain, 105(1-2), 57-64.

von Dincklage, F., Hackbarth, M., Schneider, M., Baars, J. H., \& Rehberg, B. (2009). Introduction of a continual RIII reflex threshold tracking algorithm. Brain Research, 1260, 24-29.

von Dincklage, F., Olbrich, H., Baars, J. H., \& Rehberg, B. (2013). Habituation of the nociceptive flexion reflex is dependent on interstimulus interval and stimulus intensity. Journal of Clinical Neuroscience, 20(6), 848-850.

Wallis, S. A., Baker, D. H., Meese, T. S., \& Georgeson, M. A. (2013). The slope of the psychometric function and nonstationarity of thresholds in spatiotemporal contrast vision. Vision Research, 76, 1-10. 



\section{Chapter 4}

Characterization of a psychophysical method for simultaneous tracking of multiple non-stationary thresholds

Robert J. Doll, Peter H. Veltink, Jan R. Buitenweg 
Abstract Nociceptive stimuli are processed by multiple underlying mechanisms, which are continuously modulated by ascending and descending control. Psychophysical thresholds are used to characterize nociceptive function for diagnostic purposes. However, single momentary thresholds cannot distinguish between mechanisms and cannot capture non-stationarities induced by conditioning stimuli to test modulating mechanisms. In previous work, we presented stimuli with different properties allowing observation of multiple thresholds. Combining this with a threshold tracking method could allow simultaneous observation of multiple non-stationary thresholds, but may present limited estimation performance. Here, we used computer simulations to study the consequences of combining both methods on the threshold estimation performance. Psychophysical experiments were modeled in which the effect of a conditioning stimulus was represented as non-stationary psychophysical curves. The length of the tracking window and the perturbation duration and intensity were varied to determine the effects on the estimation performance. Changes in thresholds due to a modulation were observed in all simulation results. However, the threshold estimation performance was negatively affected by a non-stationary slope and when the length of the tracking windows was longer than the duration of the modulation in threshold. Existing methodology does not allow the simultaneous observation of multiple non-stationary thresholds. The methodology presented here could be used to improve the observability of multiple underlying mechanisms. The presented method can be used for simultaneous observation of multiple non-stationary thresholds. Based on the expected duration of the effect of a modulation, guidelines are provided for psychophysical experiments. 


\section{INTRODUCTION}

Psychophysical threshold estimates can be used to describe the current state of sensory function. For clinical purposes, this single estimate is used as a quantifier of disease, such as in studies of audiology (e.g., McFadden, 1983), vision (e.g., Chauhan, Tompkins, LeBlanc, \& McCormick, 1993; Wallis, Baker, Meese, \& Georgeson, 2013), and pain (e.g., Sandkühler, 2009). In a yes-no detection experiment, where stimuli with varying amplitudes are presented to a subject and the corresponding responses (i.e., detected or not detected) are recorded, the threshold is defined as the stimulus strength or amplitude resulting in a 50\% detection probability. Prior to the detection of a stimulus, for example an electrical nociceptive stimulus, the induced neural activity is processed by several peripheral (Mendell, 2011) and central mechanisms (Sandkühler, 2009; Woolf, 2011) which modulate the perceived strength and quality of the stimulus and play an important role in chronic pain disorders. However, while a single threshold can be used as an indication of the current state of underlying mechanisms, such as hyperalgesia (Treede, Meyer, Raja, \& Campbell, 1992), it cannot be used to differentiate between the different mechanisms.

In many psychophysical methods for studying sensory function, varying the stimulus properties, such as the intensity or duration, affects the processing of that stimulus and, therefore, results in a different threshold estimate. For example, an audiogram can be obtained by presenting tones with varying frequencies and intensities to a human subject. This audiogram then describes the relation between temporal properties of the stimulus (i.e., the frequency of the tone) and the hearing threshold. Hence, it provides information about the functioning of underlying mechanisms, especially those involved in transduction of sounds from the tympanic membrane into neural activity in the cochlear nerve. Similarly in the field of pain and nociception, activation of the neural processes underlying the psychophysical threshold, such as activation of peripheral nerves, neural processing in the dorsal horn and supraspinal nociceptive pathways, have been demonstrated to depend on the temporal properties of (electrical) stimuli (Giffin, Katsarava, Pfundstein, Ellrich, \& Kaube, 2004; Mouraux, Marot, \& Legrain, 2014; van der Heide, Buitenweg, Marani, \& Rutten, 2009). However, while it is common in the field of audiology to apply stimuli with varying temporal properties, it is not commonly used for studies in pain.

Stimuli which are known to activate nociceptive mechanisms include heat, electrical, mechanical, and chemical stimuli (Pud, Granovsky, \& Yarnitsky, 2009). However, the advantage of electrical stimulation is the possibility to accurately control the stimulus 
timing. This makes electrical stimulation an interesting method when fast underlying mechanisms are of interest. Electrical stimuli using an intra-epidermal needle electrode can be used for nociceptive specific stimulation (Inui \& Kakigi, 2012; Mouraux, Iannetti, \& Plaghki, 2010). From these stimuli and corresponding responses, a single threshold estimate can be obtained (see Kingdom and Prins (2009) for an introduction in psychophysical methods).

Presenting stimuli with different temporal properties in a single experiment might help observe multiple related processes, as we did in Chapter 5. Within a single experiment, simultaneous recording of responses to stimuli with different temporal properties could help observe the mechanisms involved in the nociceptive system in more detail. Similar to the work by Gracely, Lota, Walter, and Dubner (1988), different types of stimuli can be presented within a single experiment in a random order. Stimuli with a fixed number of different temporal properties are presented to subjects in a random intermingled sequence. Figure 4-1A presents an example in which stimuli, coming from three different stimulus properties, are randomly intermingled. Each combination of stimulus-response pair (SRP) corresponding to a specific combination of properties can be used to estimate a psychophysical function (Figure 4-1B).

Besides the dependency on stimulus properties, thresholds can show continuous and temporary changes over time as well. Continuous effects can be caused by phenomena such as fatigue, loss of attention, and habituation effects, all of which are likely due to repetitive stimulation (Fründ, Haenel, \& Wichmann, 2011; Leek, Hanna, \& Marshall, 1991). Temporary changes in the nociceptive threshold can also be caused by an experimental perturbation (Chapter 2; Pud et al., 2009). For example, the cold pressor test is widely used for inducing central changes in the nociceptive system to study endogenous inhibition (Mitchell, MacDonald, \& Brodie, 2004; Pud et al., 2009; Talbot, Duncan, Bushnell, \& Boyer, 1987). During the cold pressor test, subjects immerse an extremity into cold water for a short period of time, which induces pain. As a result of this painful stimulus, thresholds are expected to show changes lasting for several minutes (e.g., Van Wijk \& Veldhuijzen, 2010).

One of the options to observe changes in thresholds over time is by using a tracking paradigm (von Dincklage, Hackbarth, Schneider, Baars, \& Rehberg, 2009). Instead of using all available SRPs within an experiment to obtain a single threshold estimate, a tracking window including a limited number of SRPs, shifts over time to estimate momentary thresholds. While the limited number of included SRPs for estimations will 


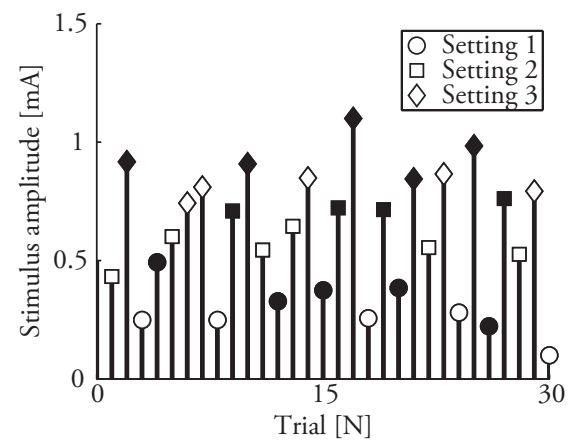

A

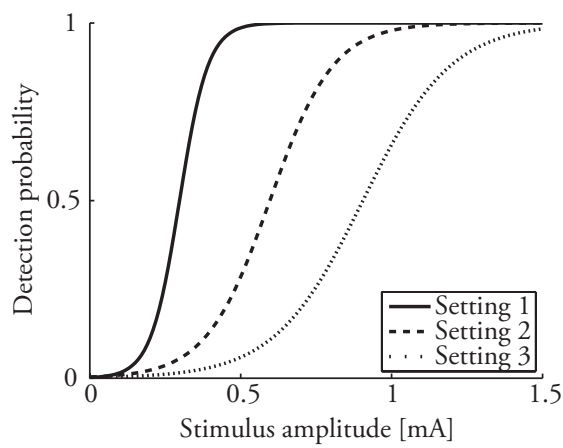

B

Figure 4-1 (A) A typical example of an experiment where stimuli with three different combinations of properties are presented in a random intermingled order. Open and closed markers indicate non-detected and detected stimuli, respectively. (B) A psychophysical function per combination of stimulus properties can be estimated using the stimulus response pairs coming from the experiment.

result in a lower estimation precision, it does capture both threshold drift and changes induced by the cold pressor test (Chapter 2 and Chapter 3).

Combining the tracking paradigm with the multiple threshold estimation procedure allows the observation of both stimulus and time dependent properties. However, as can be seen in Figure 4-1A, the inter-stimulus interval (ISI) between stimuli of the same combination of temporal properties increases when multiple thresholds are tracked within a single experiment. For example, when stimuli are applied at a rate of two seconds per stimulus, the average ISI increases from 2 to 6 seconds when three thresholds are tracked. Then, the total length of the tracking window increases when the number of SRPs is kept constant for each window. As a result, observability of changing thresholds could be impaired when more thresholds are tracked.

For illustrative purposes, we present a subset from an unpublished experiment we performed. The Medical Ethics Committee Twente approved all experimental procedures. For this subset, 14 healthy human subjects were included in a psychophysical yes-no detection task. Two combinations of temporal parameters were used for electrical nociceptive stimulation using a needle electrode (Chapter 2; Steenbergen et al., 2012). The first stimulus type was a single pulse with a pulse-width of $420[\mu \mathrm{s}]$, and the second stimulus type was a two pulse stimulus with an inter-pulse interval of $10[\mathrm{~ms}]$ and a pulse-width of 420 [us]. Five minutes after the start of the experiment, a cold pressor test was applied for two minutes. Momentary threshold estimates were based on 25 SRPs. Figure 4-2 presents the threshold tracks for both stimulus types. Both thresholds are different and increase over time during the cold pressor. Moreover, both thresholds 


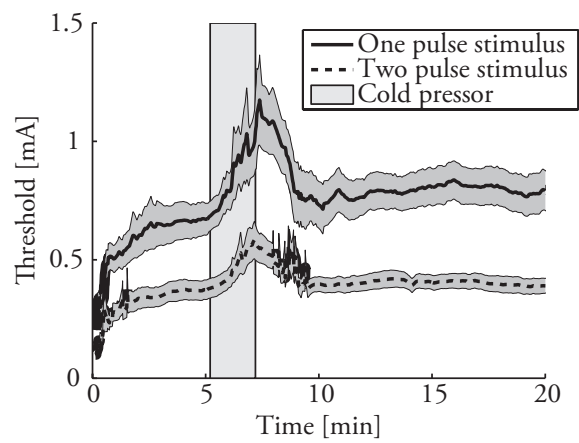

Figure 4-2 Estimated threshold tracks $( \pm S E)$ of one and two pulse stimuli over time in 14 healthy human subjects. Stimuli were presented in a random intermingeld order, such as in Figure 4-1. A cold pressor test was performed for two minutes.

increased over time during the cold pressor and decreased after its termination. However, a prolonged elevation was observed in both tracks suggesting a sustained cold pressor effect for up to 2 to 3 minutes. It is not immediately clear whether this prolonged increase is due to an actual increased threshold, or due to limitations of the tracking methodology (e.g., due to the length of the tracking window). While the observability of tracking a single threshold during a non-stationary threshold was studied in Chapter 2 and Chapter 3, the observability of tracking multiple non-stationary thresholds was not studied before. Therefore, the aim of this study is to observe how simultaneously tracking multiple non-stationary thresholds affects the estimation quality and to provide guidelines on how to implement a multiple threshold tracking experiment.

We used a Monte Carlo simulation approach to study how the threshold tracking performance, in terms of estimation bias and precision, is affected by a non-stationary psychophysical function. A psychophysical experiment was modelled in which several experimental and subject variables were varied to characterize the multiple threshold tracking paradigm. The ISI was varied such that the results would represent the results of tracking one, three, or five thresholds. Moreover, the number of SRPs in the tracking window was varied as well. The effect of a perturbation stimulus was modeled as a stepfunction or a ramp in the threshold or slope, or as a temporary increase in the threshold with varying amplitudes and durations. 


\section{METHODS}

Threshold estimates over time, coming from threshold tracks, were compared by means of Monte Carlo simulations. The simulation procedures described below are similar to the procedures described in Chapter 2 and Chapter 3. Several realizations of a 15 minute psychophysical experiment were simulated using a stochastic psychophysical model to simulate the responses to stimulus amplitudes. The characterization of the threshold tracking paradigm was done in three parts: (1) varying parameters specific to the tracking paradigm (i.e., ISI and number of SRPs in the tracking window), and (2) varying subject specific parameters (i.e., the slope of the psychophysical function), and (3) varying experiment and subject specific parameters related to perturbations (i.e., the amplitude and duration of the perturbation). For each experiment presented in this paper, 10.000 realizations were simulated. All simulations and analysis were performed with MATLAB 8.1. (MathWorks, Inc., Natick, Massachusetts, U.S.A.).

\section{Psychophysical model and model parameters}

The probability $p$ of detecting a stimulus of amplitude $x[\mathrm{~mA}]$ was modeled with a logistic psychometric function:

$$
p(x ; \alpha(t), \beta(t))=(1+\exp (\beta(t)[\alpha(t)-x]))^{-1}
$$

where $\alpha(t)$ and $\beta(t)$ were the threshold and slope parameter of the psychometric function, respectively.

In this study, we chose our model parameters such that they would be similar to the results found in our human subject experiments. In an experiment described in a previous study, we recorded SRPs to electrical stimuli for 22 minutes (Chapter 2). Stimuli were presented with a rate of about 1.5 to 3.5 seconds per stimulus. After the first 5 minutes, subjects were instructed to immerse their hand into cold water that effected in a painful sensation for a maximum of 3 minutes. The threshold prior to hand immersion was found to be between about 0.2 and $0.4 \mathrm{~mA}$. During the immersion, the threshold increased with about $0.3 \mathrm{~mA}$. Specific model parameters can be found in the paragraphs below.

\section{Effect of ISI and number of SRPs in the tracking window}

To study the effect of ISI and number of SRPs in the tracking window, the slope parameter $\beta(t)$ was fixed at $20\left[(\mathrm{~mA})^{-1}\right]$ throughout the experiment and the threshold parameter $\alpha(t)$ was defined as $0.3[\mathrm{~mA}]$ for the first 5 minutes. The threshold was 


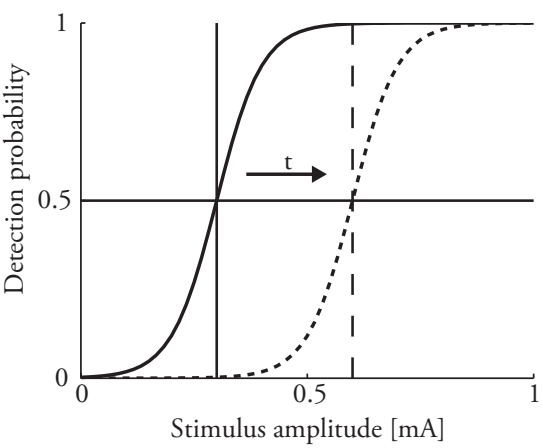

A

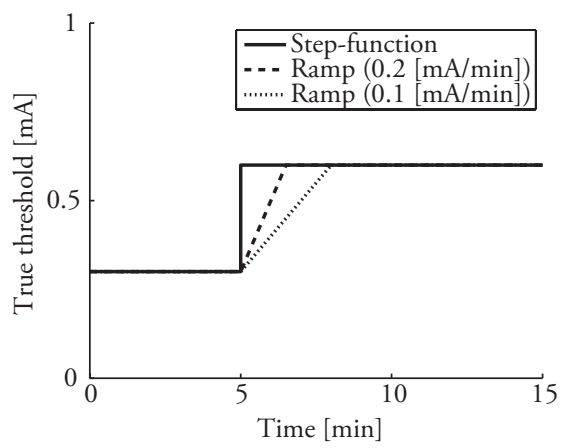

B

Figure 4-3 (A) Simulated true psychophysical functions. The threshold is defined as the stimulus necessary to elicit a .5 detection probability and shifts over time from $0.3[\mathrm{~mA}]$ to $0.6[\mathrm{~mA}]$. (B) The true threshold shifts over time from the $0.3[\mathrm{~mA}]$ to the pertubed threshold $(0.6[\mathrm{~mA}])$ after 5 minutes. Either a step-function or a ramp (of either 0.2 or $0.2[(\mathrm{~mA}) / \mathrm{min}])$ was used as the transition between unperturbed and pertubed psychophysical function.

modeled as either a step-function, or as a ramp-function. The psychophysical functions at different time-points and the threshold over time are illustrated in Figure 4-3A and Figure 4-3B, respectively.

To study the effect of the ISI, the number of SRPs in the tracking window was fixed at 25 while the ISI was 2,6 , or 10 [s]. The ISI values were chosen such that they would represent similar values as when tracking one, three, or five thresholds in our experimental paradigm. To study the effect of the number of SRPs in the tracking window, the ISI was fixed at 6 [s] and the tracking window included either 15, 25, or 35 SRPs. Given the ISI and number of SRPs, the total length of the tracking window can be calculated by multiplying the number of SRPs by the ISI.

\section{Effect of a non-stationary slope}

To study the effect of a non-stationary slope two cases were studied: (1) the threshold parameter $\alpha(t)$ was fixed at $0.3[\mathrm{~mA}]$ and the slope parameter $\beta(t)$ was either a step-function, or a ramp function, and (2) the threshold parameter $\alpha(t)$ was modeled as a step-function as in Figure 4-3 and the slope parameter $\beta(t)$ as negative step-function. Figure 4-4A illustrates the psychophysical functions with varying slopes and thresholds. The time-course of the slopes are illustrated in Figure 4-4B. The ISI was set to 6 [s] and the tracking window contained 25 SRPs for threshold estimation. 


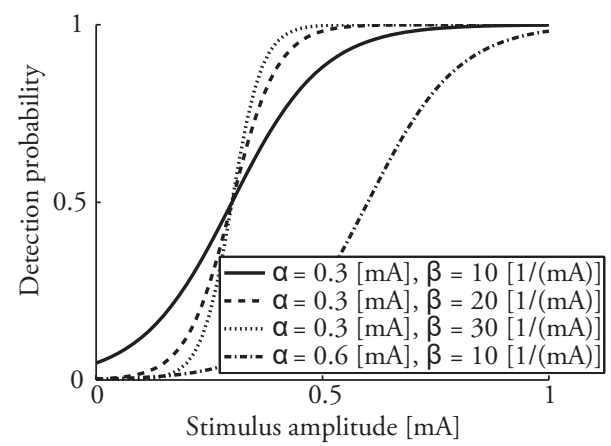

A

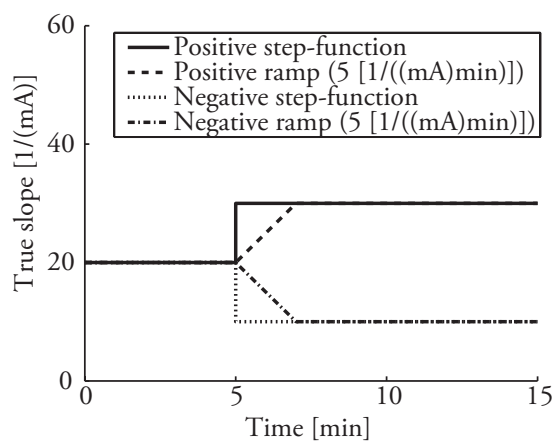

B

Figure 4-4 (A) Simulated true psychophysical functions. The default function has a slope of $20[1 /(\mathrm{mA})]$ and a higher threshold of $0.3[\mathrm{~mA}]$ and shifts over time to a function with a steeper or shallower slope, or with a shallower slope and higher threshold. (B) The slope of the psychophysical function shifts over time from 20 to either 10 or 30 [1/(mA)] with a step-function or a rate of $5[1 /((\mathrm{mA}) / \mathrm{min})]$.

\section{Effect of perturbation amplitude and duration}

To study the effect of the amplitude and duration of a perturbation, the slope parameter $\beta(t)$ was fixed at $20\left[(\mathrm{~mA})^{-1}\right]$ and the threshold $\alpha(t)$ was defined as:

$$
\alpha(t)= \begin{cases}0.3, & 0<t \leq 5 \\ \alpha_{1}, & 5<t \leq t_{1}, \\ 0.3, & t_{1}<t \leq 15\end{cases}
$$

where $\alpha(t)$ the threshold during the perturbation, and $t$ the time in minutes. The psychophysical functions at different time-points and the threshold over time are illustrated in Figure 4-5A and Figure 4-5B, respectively. To study the effect of the perturbation amplitude, the threshold during the perturbation $\alpha(t)$ was set at either $0.4,0.6$, or $0.8[\mathrm{~mA}]$ while $t_{1}$ was set at $8[\mathrm{~min}]$. To study the effect of the perturbation duration, $t_{1}$ was set at either 6 , 8 , or 10 [min] while the threshold during the perturbation $\alpha(t)$ was set at $0.6[\mathrm{~mA}]$. The ISI was set to $6[\mathrm{~s}]$ and the tracking window contained 25 SRPs for threshold estimation.

\section{Stimulus selection and threshold tracking}

For probing the modeled psychophysical functions by SRPs, stimulus amplitudes $\mathrm{x}$, were selected according to an adaptive probing procedure (Chapter 2 and Chapter 3). The procedure started with a pre-defined set of 5 equidistant stimulus amplitudes between 0 and $0.4 \mathrm{~mA}$, from which new stimulus amplitudes were randomly selected. All amplitudes in the set were increased by a step size of $0.1 \mathrm{~mA}$ after a not-detected 


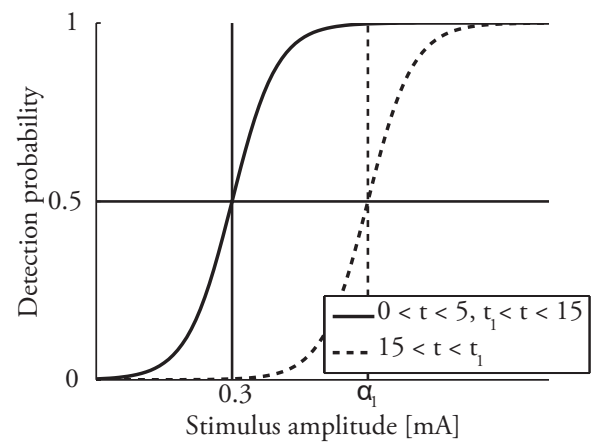

A

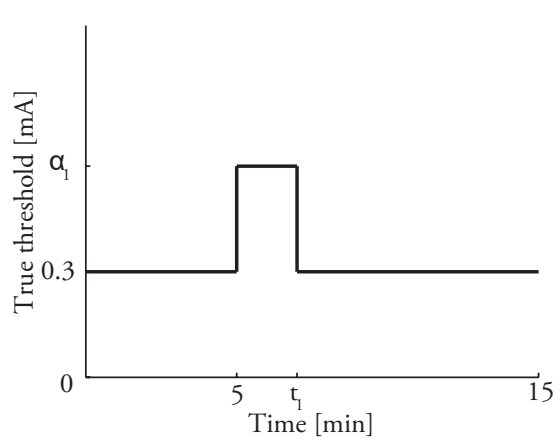

B

Figure 4-5 (A) Simulated true psychophysical functions. (B) The true threshold is shifted from the initial threshold 0.3 $[\mathrm{mA}]$ to the threshold during the perturbation $\alpha_{1}[\mathrm{~mA}]$ after 5 minutes and returns to the initial threshold at time point $\mathrm{t}_{1}$

stimulus and decreased with $0.1 \mathrm{~mA}$ after a detected stimulus. This procedure selects stimulus such that the amplitude were near the detection threshold. For an illustration of the stimulus selection procedure, see Figure 3-2B in (Chapter 3).

Thresholds were tracked by applying logistic regression over a shifting window. Momentary threshold estimates were based on including a fixed number of preceding stimulus-response pairs (see sections 2.1.1 to 2.1.3 for more details on the number of stimulus-response pairs). The stimulation times (i.e., the moments at which stimuli were presented) were included as covariate in the logistic model (Chapter 3). More details on how a single threshold is tracked can be found in Figure 2-1D-F (Chapter 2).

\section{Analysis}

Threshold estimates were only included for analysis when the momentary threshold estimate was between 0 and $5 \mathrm{~mA}$. All other momentary estimates were considered unrealistic and therefore excluded from the dataset. This resulted in that less than $2.5 \%$ of all momentary threshold estimates were removed prior to the analysis. The bias and precision of threshold estimates over time were determined in all simulations. The bias was defined as the mean difference between the true and the estimated threshold and the precision as the reciprocal of the variance of threshold estimates (Treutwein, 1995).

\section{RESULTS}

\section{Effect of ISI and number of SRPs in the tracking window}

The means and standard deviations, estimation biases, and estimation precisions of the threshold tracks while varying the ISI and number of SRPs in the tracking window are presented in Figure 4-6 and Figure 4-7, respectively. A start-up effect can be seen in all 
simulation results. The threshold estimates show a transient change during the first few minutes of the experiment. This start-up effect was reflected in both the bias and the precision. Overall, estimates were of negligible bias within one to two minutes. However, the time required before the estimation precision became stable was dependent on the window length: shorter ISI and fewer SRPs resulted in a faster rate of convergence, and longer ISI and more SRPs resulted in a slower rate of convergence. Only the number of SRPs included in the tracking window affected the baseline precision value. The highest precision was found when the tracking window included 35 SRPs.

Right after the change in true threshold, threshold estimations gradually increased over time. The relative rate of convergence of estimates towards the true perturbed threshold depended on the size of the window. Shorter and longer windows resulted in faster and slower convergence, respectively. When the tracking window was relatively short, for example when the ISI was $2[\mathrm{~s}]$ and the threshold increased with a rate of $0.2[(\mathrm{~mA}) / \mathrm{min}]$ or slower, the estimation bias converged to a constant value before decreasing. Moreover, the estimation precision was less affected by slow changing thresholds than by fast changing thresholds.

\section{Effect of a non-stationary slope}

The means and standard deviations, estimation biases, and estimation precisions of the threshold tracks while the slope was non-stationary are presented in Figure 4-8. The threshold was fixed at $0.3[\mathrm{~mA}]$ in the upper row and the slope was modeled as either a step-function or as a ramp function. The threshold was modeled as a positive stepfunction and the slope as a negative step-function in the lower row.

Similar to the results presented in the previous section, a start-up effect is visible in the bias and precision. The threshold estimation bias remained unaffected by a non-stationary slope, when the threshold was fixed at $0.3[\mathrm{~mA}]$ throughout the experiment. The estimation precision, however, was affected by the non-stationary slope and converged slowly to a stable value. The value to which the precision converged depended on the slope value: steeper slopes resulted in higher estimation precisions than shallower slopes. When the threshold increased and the slope decreased, estimates were biased and of lower precision than before the change. The estimation precision converged to a lower estimation precision than before the decrease in the slope. 

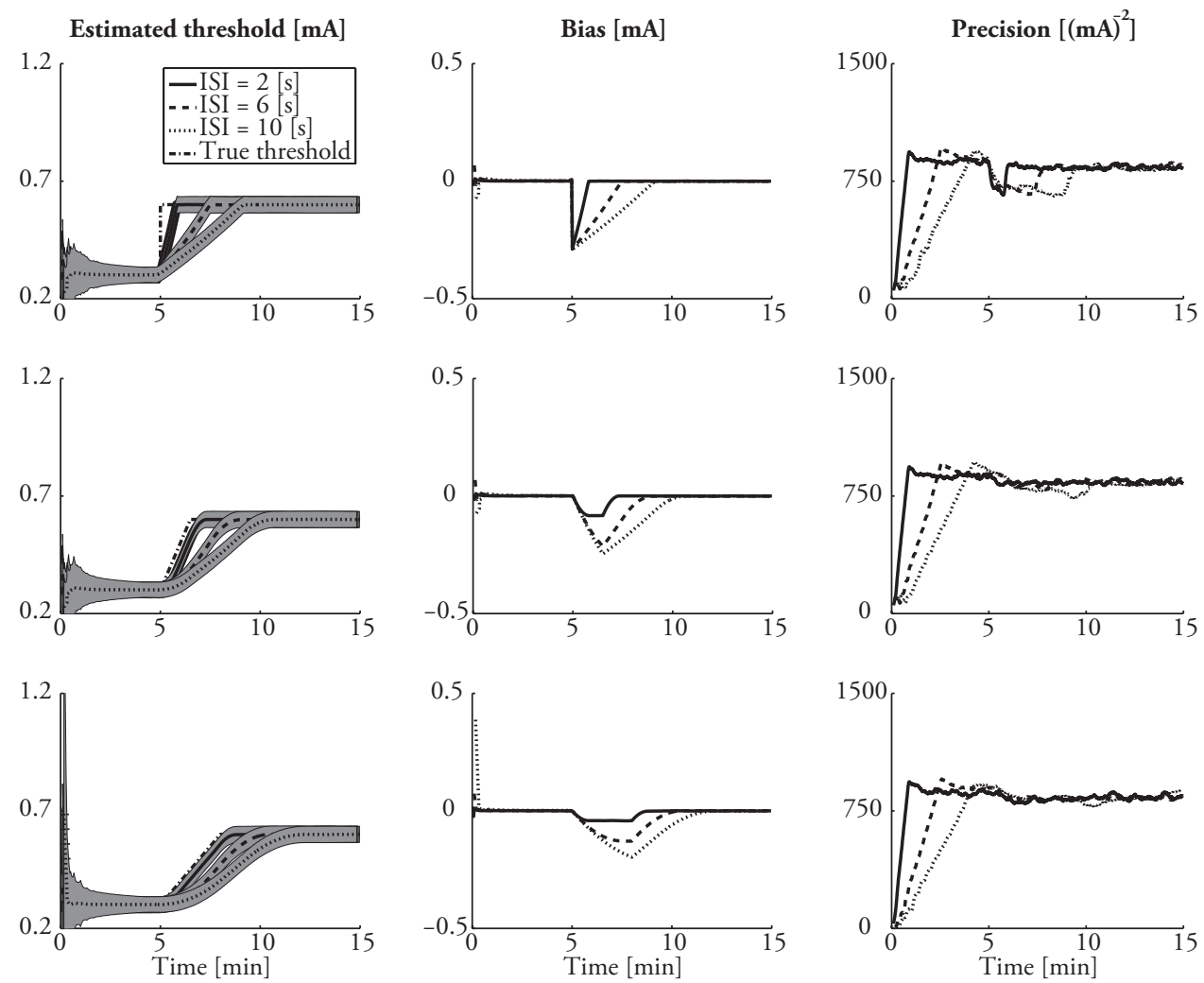

Figure 4-6 Simulation results while varying the ISI. The first column presents the mean and standard deviations of the estimated thresholds. The second and third columns present the estimation bias and precision, respectively.

\section{Effect of perturbation amplitude and duration.}

The means and standard deviations, estimation biases, and estimation precisions of the threshold tracks while varying the amplitude and duration of the perturbation are presented in Figure 4-9. Again, a start-up effect can be seen in the simulation results.

All threshold estimates were able to reach the true threshold value when the perturbation amplitude was varied. While the estimation bias was highest for the largest threshold difference, all biases became negligible right before the end of the perturbation (i.e., at after the $8^{\text {th }}$ minute), regardless of the perturbation amplitude. Moreover, the lowest precision was observed when the perturbation amplitude was largest, but all converged right before the end of the perturbation. Similar results were observed when the true threshold returned to baseline value after the perturbation. 

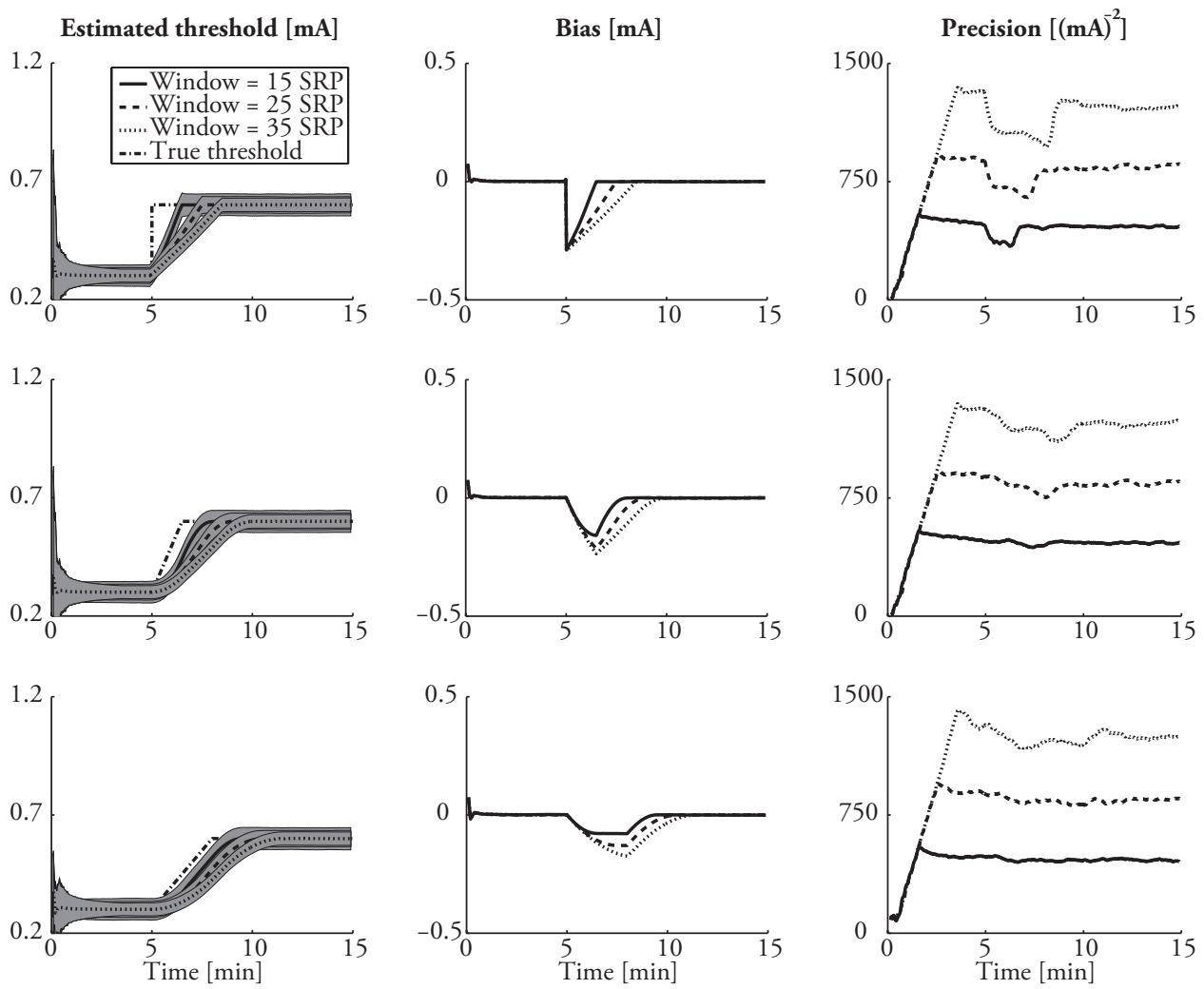

Figure 4-7 Simulation results while varying the number of SRPs in the tracking window. The first column presents the mean and standard deviations of the estimated thresholds. The second and third columns present the estimation bias and precision, respectively.

When the length of the tracking window was shorter than the duration of the perturbation (i.e., when the duration of the perturbation was set to three or five minutes), threshold tracks showed a decrease right after the perturbation was stopped and the true threshold returned to the unperturbed state. Moreover, the estimation precision dropped temporarily but returned to a stable value. However, when the length of the window was longer than the duration of the perturbation, estimates temporarily continued to increase while the true threshold already returned to baseline value. This resulted in a temporary plateau in threshold estimates. The plateau lasted for about 2 minutes when the perturbation duration was set to 1 minute. Additionally, as the estimation precision did not reach a stable value before the end of the perturbation, the precision dropped further before increasing again until a stable value was reached. 

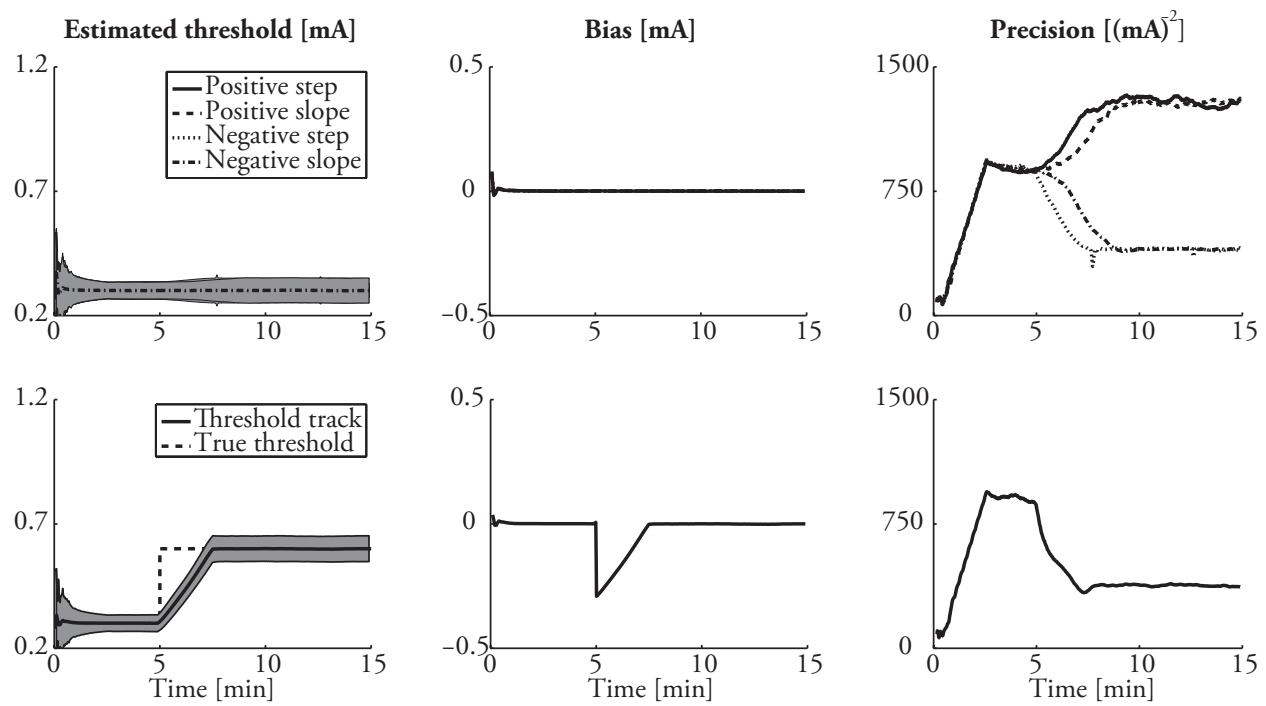

Figure 4-8 Simulation results while varying the slope. The first column presents the mean and standard deviations of the estimated thresholds. The second and third columns present the estimation bias and precision, respectively. The threshold was fixed to $0.3[\mathrm{~mA}]$ in the first row and the slope was modeled as either a step-function or as a ramp function.

\section{DISCUSSION}

In this study, a Monte Carlo simulation approach was used to study how the threshold tracking performance, in terms of estimation bias and precision, is affected by a non-stationary psychophysical function. A psychophysical experiment was modeled in which several experimental and subject variables were varied to characterize the performance of the multiple threshold tracking paradigm. The ISI was varied such that the results would represent the results of tracking one, three, or five thresholds. Moreover, the number of SRPs in the tracking window was varied as well.

In this study, a nociceptive perturbation was represented by various realizations of non-stationary psychophysical functions. First, a step-function and a ramp-function in the threshold were used to characterize the parameters of the tracking paradigm (i.e., the ISI and number of SRPs in the tracking window). Second, a step-function and a ramp-function in the slope or in the threshold and slope were used to observe effects of non-stationary slopes. Finally, a block function was used to observe the effect of the amplitude and duration of a nociceptive perturbation on threshold estimates.

A start-up effect was observed in all simulation results: all threshold estimates were biased and of low precision. The estimation bias converged to a negligible bias within one to two minutes and was independent of the varied parameters affecting the window 

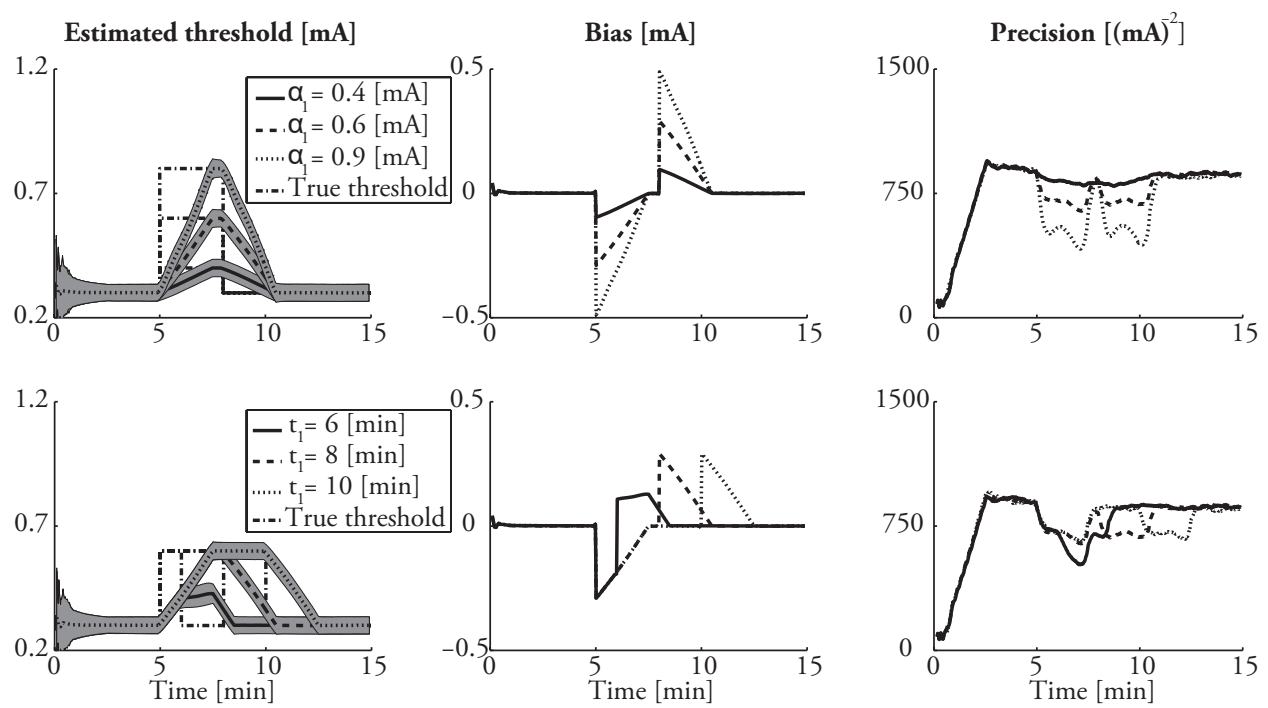

Figure 4-9 Simulation results while varying the amplitude and duration of the perturbation. The first column presents the mean and standard deviations of the estimated thresholds. The second and third columns present the estimation bias and precision, respectively. The first and second row present the results when varying the amplitude and duration of the perturbation, respectively.

length (i.e., ISI and number of SRPs in the tracking window). However, the time required for the estimation precision to converge to a stable value did depend on the ISI and number of SRPs in the tracking window. It took about the length of the tracking window before the precision converged to a stable value. Therefore, both longer ISI values and more SRPs in the tracking window negatively affected this start-up effect. As a result, threshold estimates at the beginning of a psychophysical experiment are imprecise for the duration of a tracking window (i.e., the number of SRPs times the ISI) and one should be cautious when interpreting the estimates.

Right after the change in true threshold, threshold estimates started to increase in all simulation results. Moreover, the estimation precision dropped right after the change in threshold. The time required to reach the true value of the perturbed threshold with a stable estimation precision depended on the length of the tracking window. The shortest tracking window converged fastest while the longest tracking window converged slowest. Moreover, the bias after a slow changing threshold (i.e., with a ramp of 0.2 or $0.1[(\mathrm{~mA}) / \mathrm{min}])$ short tracking windows converges to a constant value. The value to which the bias converged depended on the length of the window and on the rate with which the true threshold increased. Lowest biases were observed for short window lengths and slowly increasing true thresholds. Moreover, the constant bias value means that threshold tracks at that time-point run parallel to the true threshold. This bias 
is mainly because momentary threshold estimates are only based on previous SRPs. Basing momentary thresholds on past and future SRPs reduce estimation bias caused by drifting thresholds (Chapter 3).

When the threshold was fixed, and the slope was modeled as either a step-function or as a ramp function, only a decrease in estimation precision was observed. The bias was not affected by the change in slope. When both the true threshold and true slope were non-stationary, threshold estimates were underestimated and showed a similar pattern as when only the true threshold was non-stationary. Moreover, the estimation precision decreased and converged to a similar precision value as when only the slope was non-stationary. Therefore, slope non-stationarities only affect the threshold estimation precision.

To observe the effect of the duration and amplitude of a nociceptive perturbation, the threshold was modeled as a block function. The amplitude of the perturbation affected both the estimation bias and estimation precision. Larger amplitudes resulted in larger biases and lower precisions. However, the bias returned to zero and the precision converged to baseline values within the same time. Again, this time was similar to the length of the tracking window.

When comparing the results between different perturbation durations, the maximum amplitude of true threshold was only reached when the length of the window was shorter than the duration of the perturbation. Estimates coming from tracking windows of which the length was longer than the duration of the perturbation showed a temporary plateau in estimations right after the true threshold returned to its unperturbed value. These overestimations are caused by the inclusion of SRPs obtained prior, during, and after the perturbation. Therefore, the threshold estimates after the perturbation were temporarily also based on the true threshold before and during the perturbation. In practice, the long-lasting bias after a perturbation could be interpreted as if the threshold was actually increased for a prolonged time. Therefore, when thresholds are expected to change temporarily due to a perturbation (e.g., due to a cold pressor test), one should ensure that the time length of the tracking window is shorter than the expected effect of the perturbation. Otherwise, estimations remain biased and of lower precision throughout the duration of the perturbation and could hamper inferences on the dynamics of the perturbation. 
One of the options to prevent the above mentioned misinterpretation of the threshold estimates is to increase the duration of the perturbation. However, a long painful perturbation might be intolerable for some subjects and is therefore not always a suitable option. Another option is to shorten the tracking window. Shortening the ISI or reducing the number of SRPs in the tracking window are two options which shorten the tracking window. In our simulations, we fixed the ISI at 2, 6, or 10 seconds. Given 25 SRPs, this would result in a length of about 50, 150, or 250 seconds. These values were chosen such that these would be similar to tracking a single, three, or five thresholds belonging to different stimulus types in our experimental paradigm. Reduction of the ISI in our experimental paradigm can be achieved by either increasing the rate at which stimuli are applied, or reducing the number of threshold tracks. Whether reducing the rate at which stimuli are applied is a possibility depends on the time subjects need to respond to a stimulus. Moreover, habituation processes might play a more substantial role when the ISI is decreased (von Dincklage, Olbrich, Baars, \& Rehberg, 2013). Reducing the ISI by reducing the number of threshold tracks would improve the observability of the dynamics of a perturbation. However, it does reduce the observability of multiple underlying mechanisms. The last suggestion of reducing the window length is by reducing the number of SRPs in the tracking window. This will result in faster responses to changes in the threshold and faster convergence towards true threshold values. However, as fewer data-points are available for threshold estimation, the estimation precision decreases. Therefore, a trade-off must be made between the observability of the dynamics and estimation precision.

Considering the illustrative data from the psychophysical experiment presented in the introduction (Figure 4-2), it can still be argued that the threshold increased during the cold pressor test for both parameter types (i.e., the one pulse and the two pulse stimulus). Both threshold tracks show an increase in the detection threshold right after the start until the end of the cold pressor test. Moreover, the tracks decreased after termination of the cold pressor test and suggest a sustained cold pressor effect of 2 to 3 minutes. However, as can be seen from the simulation results, it is likely that a part of this prolonged elevation is due to the limitations of the experimental procedure. Overall, the tracks suggest that the used window of 25 SRPs was too long to fully observe the dynamics of the underlying mechanisms. Therefore, for this illustration, either the number of SRPs could be reduced, or, for future studies, the ISI could be decreased by either increasing the stimulation frequency or reducing the number of tracks to one. 
A few limitations of this study should be mentioned. First, subject-related variabilities in the psychophysical curve such as guessing or lapsing were not considered in this study. Human subjects occasionally lapse and guess during experiments and thereby affect the estimation quality when not accounted for (Prins, 2012; Wichmann \& Hill, 2001). Additionally, both lapsing and guessing rates are unlikely to remain stationary throughout an experiment, especially during a (nociceptive) perturbation. This could potentially hamper the observability of threshold dynamics. Second, responses to trials might be affected by responses given in earlier trials (Fründ, Wichmann, \& Macke, 2014). Moreover, the inter-trial dependency likely depends on the stimulus type. In our experimental paradigm, different stimulus types are presented in a pseudo-random order, complicating quantification of the inter-trial dependency. Because we did not include variabilities in parameters such as the lapsing and guessing rate, and did not include inter-trial dependencies, the results presented here reflect a best-case scenario. Future studies could study the effects of less-ideal subjects by, for example, including non-stationary lapsing and guessing rates, and inter-trial dependencies.

In conclusion, observation of multiple mechanisms might be achieved by tracking multiple thresholds to stimuli with different temporal properties. However, tracking multiple threshold increases the ISI resulting in slower tracking performance and changes in underlying mechanisms which result in fast changes of detection thresholds will negatively affect the estimation quality. Therefore, in psychophysical experiments where underlying mechanisms are expected to affect the threshold over time (e.g., due to a perturbation), the expected duration of the effect on the threshold should be considered. Based on the duration, a trade-off between the observability of multiple underlying mechanisms (i.e., the number of threshold tracks) and the estimation bias and precision can be made.

\section{REFERENCES}

Chauhan, B. C., Tompkins, J. D., LeBlanc, R. P., \& McCormick, T. A. (1993). Characteristics of frequency-of-seeing curves in normal subjects, patients with suspected glaucoma, and patients with glaucoma. Investigative Ophthalmology and Visual Science, 34(13), 3534-3540.

Fründ, I., Haenel, N. V., \& Wichmann, F. A. (2011). Inference for psychometric functions in the presence of nonstationary behavior. Journal of Vision, 11(6), 1-19.

Fründ, I., Wichmann, F. A., \& Macke, J. H. (2014). Quantifying the effect of intertrial dependence on perceptual decisions. Journal of Vision, 14(7), 1-16.

Giffin, N. J., Katsarava, Z., Pfundstein, A., Ellrich, J., \& Kaube, H. (2004). The effect of multiple stimuli on the modulation of the 'nociceptive' blink reflex. Pain, 108(1-2), 124-128.

Gracely, R. H., Lota, L., Walter, D. J., \& Dubner, R. (1988). A multiple random staircase method of psychophysical pain assessment. Pain, 32(1), 55-63. 
Inui, K., \& Kakigi, R. (2012). Pain perception in humans: use of intraepidermal electrical stimulation. Journal of Neurology, Neurosurgery and Psychiatry, 83(5), 551-556.

Kingdom, F. A. A., \& Prins, N. (2009). Psychophysics: A Practical Introduction: Elsevier Science \& Technology.

Leek, M. R., Hanna, T. E., \& Marshall, L. (1991). An interleaved tracking procedure to monitor unstable psychometric functions. Journal of the Acoustical Society of America, 90(3), 1385-1397.

McFadden, D. F. L. (1983). Audition: Some relations between normal and pathalogical hearing. Annual Review of Psychology, 34(1), 95.

Mendell, L. M. (2011). Computational functions of neurons and circuits signaling injury: Relationship to pain behavior. Proceedings of the National Academy of Sciences of the United States of America, 108(SUPPL. 3), 15596-15601.

Mitchell, L. A., MacDonald, R. A., \& Brodie, E. E. (2004). Temperature and the cold pressor test. Journal of Pain, 5(4), 233-237.

Mouraux, A., Iannetti, G. D., \& Plaghki, L. (2010). Low intensity intra-epidermal electrical stimulation can activate A $\delta$-nociceptors selectively. Pain, 150(1), 199-207.

Mouraux, A., Marot, E., \& Legrain, V. (2014). Short trains of intra-epidermal electrical stimulation to elicit reliable behavioral and electrophysiological responses to the selective activation of nociceptors in humans. Neuroscience Letters, 561, 69-73.

Prins, N. (2012). The psychometric function: The lapse rate revisited. Journal of Vision, 12(6).

Pud, D., Granovsky, Y., \& Yarnitsky, D. (2009). The methodology of experimentally induced diffuse noxious inhibitory control (DNIC)-like effect in humans. Pain, 144(1-2), 16-19.

Sandkühler, J. (2009). Models and mechanisms of hyperalgesia and allodynia. Physiological Reviews, 89(2), 707-758.

Steenbergen, P., Buitenweg, J. R., Trojan, J., van der Heide, E. M., van den Heuvel, T., Flor, H., \& Veltink, P. H. (2012). A system for inducing concurrent tactile and nociceptive sensations at the same site using electrocutaneous stimulation. Behavior Research Methods, 44(4), 924-933.

Talbot, J. D., Duncan, G. H., Bushnell, M. C., \& Boyer, M. (1987). Diffuse noxious inhibitory controls (DNICs): psychophysical evidence in man for intersegmental suppression of noxious heat perception by cold pressor pain. Pain, 30(2), 221-232.

Treede, R. D., Meyer, R. A., Raja, S. N., \& Campbell, J. N. (1992). Peripheral and central mechanisms of cutaneous hyperalgesia. Progress in Neurobiology, 38(4), 397-421.

Treutwein, B. (1995). Adaptive psychophysical procedures. Vision Research, 35(17), 2503-2522.

van der Heide, E. M., Buitenweg, J. R., Marani, E., \& Rutten, W. L. (2009). Single pulse and pulse train modulation of cutaneous electrical stimulation: a comparison of methods. Journal of Clinical Neurophysiology, 26(1), 54-60.

Van Wijk, G., \& Veldhuijzen, D. S. (2010). Perspective on diffuse noxious inhibitory controls as a model of endogenous pain modulation in clinical pain syndromes. Journal of Pain, 11(5), 408-419.

von Dincklage, F., Hackbarth, M., Schneider, M., Baars, J. H., \& Rehberg, B. (2009). Introduction of a continual RIII reflex threshold tracking algorithm. Brain Research, 1260, 24-29.

von Dincklage, F., Olbrich, H., Baars, J. H., \& Rehberg, B. (2013). Habituation of the nociceptive flexion reflex is dependent on inter-stimulus interval and stimulus intensity. Journal of Clinical Neuroscience, 20(6), 848-850.

Wallis, S. A., Baker, D. H., Meese, T. S., \& Georgeson, M. A. (2013). The slope of the psychometric function and nonstationarity of thresholds in spatiotemporal contrast vision. Vision Research, 76(0), 1-10.

Wichmann, F. A., \& Hill, N. J. (2001). The psychometric function: II. Bootstrap-based confidence intervals and sampling. Perception and Psychophysics, 63(8), 1314-1329.

Woolf, C. J. (2011). Central sensitization: Implications for the diagnosis and treatment of pain. Pain, 152(SUPPL.3), S2-S15. 



\section{Observation of nociceptive processing using electrocutaneous stimulation}





\section{Chapter 5}

Effect of temporal stimulus properties on the nociceptive detection probability using intra-epidermal electrical stimulation

Robert J. Doll, Annefloor C. A. Maten, Sjoerd P. G. Spaan, Peter H. Veltink, Jan R. Buitenweg

Experimental Brain Research (2016) 234(1), 219-227 
Abstract Chronic pain disorders can be initiated and maintained by malfunctioning of one or several mechanisms underlying the nociceptive function. Although several quantitative sensory testing methods exist to characterize the nociceptive function, it remains difficult to distinguish the contributions of individual mechanisms. Intra-epidermal electrical stimulation of nociceptive fibers allows defining stimuli with temporal properties within the timescale of these mechanisms. Here, we studied the effect of stimulus properties on the psychophysical detection probability. A psychophysical detection experiment was conducted including 30 healthy human subjects. Subjects were presented with electrical stimuli having various temporal properties. The pulse-width was varied for single-pulse stimuli (either 420 or $840 \mu \mathrm{s}$ ) and the inter-pulse interval for double-pulse stimuli $(10,50$, or $100 \mathrm{~ms})$. Generalized linear mixed models were used to obtain estimates of thresholds and slopes of the psychophysical function. The $840 \mu$ s single-pulse resulted in a lower threshold and steeper slope of the psychophysical function than the 420 $\mu$ single-pulse. Moreover, a double-pulse stimulus resulted in a lower threshold and steeper slope than for single-pulse stimuli. The slopes were similar between the doublepulse stimuli, but thresholds slightly increased with increasing inter-pulse intervals. In the present study, it was demonstrated that varying the temporal properties of intraepidermal electrical stimuli results in variations in nociceptive processing. The estimated thresholds and slopes corresponding to the selection of temporal properties suggest that contributions of peripheral and central nociceptive mechanisms can be reflected in psychophysical functions. 


\section{INTRODUCTION}

Chronic pain disorders can be initiated and maintained by malfunctioning of one or several mechanisms underlying the nociceptive function. Prior to the perception of a nociceptive stimulus, the induced neural activity is processed by peripheral (Mendell, 2011) and central mechanisms (Bromm \& Lorenz, 1998; Sandkühler, 2009; Woolf, 2011) which modulate the perceived strength and quality. Malfunctions of these mechanisms may lead to, for example, peripheral sensitization which is expressed by an increase in neural activity given the same stimulus strength. Another example is central sensitization which is expressed by a higher postsynaptic neural activity given the same incoming peripheral neural activity and is said to be an important factor in chronic pain disorders (Latremoliere \& Woolf, 2009).

Experimental application of stimuli and recording the responses allow the observation and quantification of nociceptive processing. Commonly used methods for observation of stimulus processing and sensory function include psychophysical quantitative sensory testing (QST) methods (Arendt-Nielsen \& Yarnitsky, 2009; Rolke et al., 2006). Multiple QST methods exist, using a broad range of stimulus types such as thermal, mechanical, or electrical. Additionally, QST can serve as a predictor of chronic pain development (Backonja et al., 2013; Wilder-Smith, Schreyer, Scheffer, \& Arendt-Nielsen, 2010). It remains, however, difficult to distinguish the individual contributions of nociceptive mechanisms, hampering the diagnosis of chronic pain disorders.

For observing the contributions of individual nociceptive processes, stimuli must have properties such that specific characteristics of these processes are elicited. Recent studies have shown that intra-epidermal electrical stimulation can preferentially activate nociceptive A $\delta$-fibers (Inui \& Kakigi, 2012; Kodaira, Inui, \& Kakigi, 2014; Mouraux, Iannetti, \& Plaghki, 2010), as long as the stimulation current is below twice the detection threshold in order to minimize the co-activation of tactile A $\beta$-fibers (Legrain \& Mouraux, 2013; Mouraux et al., 2010). This limitation becomes problematic when estimating pain thresholds and pain tolerance thresholds. However, when presenting stimuli with varying amplitudes below this limitation, and recording the corresponding detections (i.e., detected or not-detected), the nociceptive detection probability, in terms of the threshold and slope of a psychophysical curve (Klein, 2001; Treutwein, 1995), can be safely estimated.

An advantage of electrical stimulation is the accurate control of stimulation timing allowing well-defined stimuli with temporal resolutions in the order of tens of $\mu$ s. 


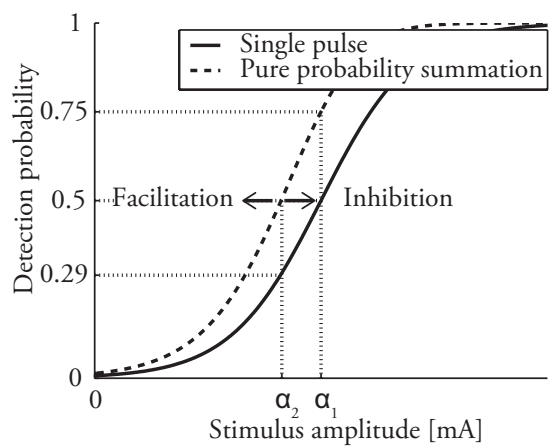

Figure 5-1 Effect of probability summation. The thick line presents the psychophysical curve for a single-pulse stimulus. The thick dashed line presents the psychophysical curve for a double-pulse stimulus, based on pure probability summation. An additional shift in the psychophysical curve can occur when the double-pulse stimulus is subject to inhibiting or facilitating processes.

Varying the temporal properties of rectangular-wave current stimuli (i.e., the pulse-width (PW), number of pulses (NoP), and inter-pulse interval (IPI)) could allow probing the nociceptive system in more detail. Peripheral phenomena, such as the strength-duration relationship (Rollman, 1969; Weiss, 1901), can be probed by varying the PW. Inhibitory and/or facilitatory processes, either at a peripheral level or at a central level, could possibly be probed by varying the NoP and IPI (Mouraux, Marot, \& Legrain, 2014; van der Heide, Buitenweg, Marani, \& Rutten, 2009).

In the case of double-pulse stimuli, it should be noted that the detection probability of a double-pulse stimulus depends on the detection probability of each of the individual pulses ( $p_{1}$ and $p_{2}$, respectively) according to probability summation (e.g., Gescheider, Berryhill, Verrillo, \& Bolanowski, 1999). The detection probability of a double-pulse stimulus can be formulated as $p_{d}=1-\left(1-p_{1}\right)\left(1-p_{2}\right)$. When the separate detection probabilities of both pulses are independent and equal, this formula reduces to $p_{d}=1-\left(1-p_{1}\right)^{2}$. We refer to the latter description as pure probability summation. Figure 5-1 illustrates the relation between the detection probability of a single-pulse stimulus and a double-pulse stimulus. Due to pure probability summation, the expected threshold for the double-pulse stimulus $\left(\alpha_{2}\right)$ would equal the amplitude resulting in a 0.29 detection probability for a single-pulse. Similarly, the threshold for a single-pulse stimulus $\left(\alpha_{1}\right)$ is equal to the amplitude resulting in a 0.75 detection probability. Moreover, the psychophysical function for double-pulse stimuli is steeper than for single-pulse stimuli. When both pulses cannot be considered independent (i.e., when the detection probability of the second pulse is altered by the presence of the first pulse, hence $p_{1} \neq p_{2}$ ), either inhibitory of facilitatory processes were activated resulting in 
a psychophysical curve which is shifted from the curve described by pure probability summation.

Presenting subjects with a mixed sequence of stimuli with different predefined temporal properties within a single experiment might allow the simultaneous observation of various contributions of nociceptive processes to stimulus processing. From the combination of stimuli and corresponding responses (i.e., detected or undetected) the detection probability per stimulus type can be described by a psychophysical function in terms of a threshold and a slope (Klein, 2001; Treutwein, 1995). While the threshold is often used to indicate altered nociceptive function (e.g., hyperalgesia (Treede, Meyer, Raja, \& Campbell, 1992)), the slope of the psychophysical function is not so often used but could provide additional information about the reliability of stimulus detection by subjects (Gold \& Ding, 2013).

While the effect of temporal stimulus parameters, such as the PW and the NoP, have been studied before on the strength and quality of perception of pain, the effect on the detection probability has not been studied before. An experiment was performed where subjects were presented with stimuli with different temporal properties using intra-epidermal electrical stimulation. We study whether different temporal properties affect the psychophysical function, and whether changes in the psychophysical function reflect contributions of nociceptive processes.

\section{METHODS}

\section{Subjects}

The effect of stimulus properties on the nociceptive detection probability was studied in two psychophysical experiments, each including 15 pain-free human subjects (14 men, 16 women; mean age $=22.0, S D=1.6$ ). The Medical Ethics Committee Twente approved all experiment procedures. All subjects provided written informed consent and were rewarded with a gift voucher after participation in the experiment.

\section{Experiment design}

Two identical psychophysical experiments were performed. Subjects visited the laboratory on two consecutive days. All procedures on the first day were repeated on the second day. An electrode was attached to the subjects' left forearm which consisted of an array of five interconnected needles and four flat interconnected electrodes with a diameter of $5 \mathrm{~mm}$ (see Figure 5-2 for a schematic representation, see (Steenbergen et al., 2012) for more details). Prior to the experiment, subjects were familiarized with 


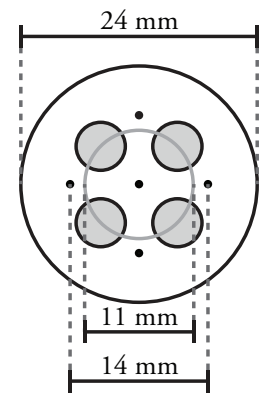

Figure 5-2 Schematic representation of the needle electrode. The electrode consists of four interconnected 5-mmdiameter disc electrodes and five interconnected needle electrodes.

the test procedures by presenting electrical stimuli with various amplitudes. Then, the 10-minute experiment started in which test-stimuli were presented to subjects.

\section{Test-stimuli}

Cathodic rectangular pulses were applied as test-stimuli using four different settings of temporal properties (Table 5-1). In the first experiment, the electrode was covered with a conducting pad that covered the flat electrodes and served as the anode. For technical reasons, a $50 \times 90 \mathrm{~mm}$ TENS electrode served as anode in the second experiment.

Four different combinations of temporal electrical properties were randomly presented during the experiment. The combinations of temporal properties were different for both experiments. For the first experiment, the PW, NoP, and IPI were varied, and in the second experiment the NoP and IPI. The electrical properties of the stimuli used in the first and second experiments are presented in Table 5-1.

Stimulus amplitudes were selected according to an adaptive probing procedure (Chapter 2). The procedure started with a predefined set of five equidistant stimulus amplitudes between 0 and $0.4 \mathrm{~mA}$ for single-pulse stimuli and between 0 and $0.2 \mathrm{~mA}$ for double-pulse stimuli. The amplitude of the upcoming stimulus was randomly selected

Table 5-1 Pulse-width (PW), number of pulses (NoP), and inter-pulse interval (IPI) used for both experiments.

\begin{tabular}{|c|c|c|c|c|c|c|}
\hline & \multicolumn{3}{|c|}{ Experiment 1} & \multicolumn{3}{|c|}{ Experiment 2} \\
\hline & $\mathrm{PW}[\mu \mathrm{s}]$ & NoP & IPI [ms] & $\mathrm{PW}[\mu \mathrm{s}]$ & NoP & IPI [ms] \\
\hline Setting 1 & 420 & 1 & - & 420 & 1 & - \\
\hline Setting 2 & 840 & 1 & - & 420 & 2 & 10 \\
\hline Setting 3 & 420 & 2 & 10 & 420 & 2 & 50 \\
\hline Setting 4 & 420 & 2 & 50 & 420 & 2 & 100 \\
\hline
\end{tabular}


from this set. All amplitudes in the set were increased and decreased with a fixed step size after a not-detected stimulus and detected stimulus, respectively. The step size was $0.1 \mathrm{~mA}$ for single-pulse stimuli and $0.05 \mathrm{~mA}$ for double-pulse stimuli. The different stimulus settings were presented in a randomly intermingled sequence.

Subjects were instructed to indicate whether they detected a stimulus by releasing a response button and to press the button again after about a second. Due to this, the interstimulus interval after a detected stimulus was longer than after a not-detected stimulus. Besides the fixed inter-stimulus interval, an extra random interval between 0.6 and $1 \mathrm{~s}$ was added to reduce the stimulation predictability. This resulted in a mean inter-stimulus interval of 2.7 and $3.5 \mathrm{~s}$ after a not-detected stimulus and after a detected stimulus, respectively. A custom computer program (written in LabVIEW 2011, SP1) controlled all stimulation procedures, as well as the registration of stimulus amplitudes in $\mathrm{mA}$, stimulation times in milliseconds, and responses to stimuli (i.e., detected or not-detected).

\section{Statistical analysis}

All data preparation was performed in MATLAB 8.1 (MathWorks, Inc., Natick, Massachusetts, U.S.A). Statistical modelling was performed using the lme4 library (Bates, Maechler, Bolker, \& Walker, 2014) in the R software package (R Core Team, 2014). For each of the two experiments, a generalized linear mixed model (GLMM) using a logit link function was built to estimate the detection probability given the stimulus amplitudes. The intercept, stimulus amplitude $[\mathrm{mA}]$, study day, setting, stimulation time $[\mathrm{s}]$, and the interaction between amplitude and setting were included as fixed effects. Between-subjects random effects were included for the intercept, stimulus amplitude, study day, setting, and stimulation time. An unstructured covariance matrix was used to model the random effects. The stimulation time variable was centered and scaled prior to analysis to speed up the estimation process.

Type III Wald Chi-square statistics were used to test the main and interaction effects of the fixed effects. Confidence intervals of the regression parameters were based on the Wald z statistics. Threshold estimates were obtained from the regression parameters, and corresponding standard errors were approximated using the Delta procedure (Faraggi, Izikson, \& Reiser, 2003; Moscatelli, Mezzetti, \& Lacquaniti, 2012). The slope estimates were obtained from the regression parameters and are equal to the estimated log-odds of the stimulus amplitude and corresponding interactions with setting. Post hoc multiple comparisons between thresholds and slopes were performed using Bonferroni $p$-value corrections. 


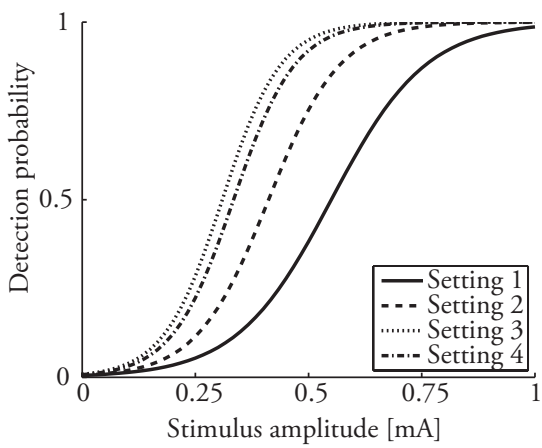

A

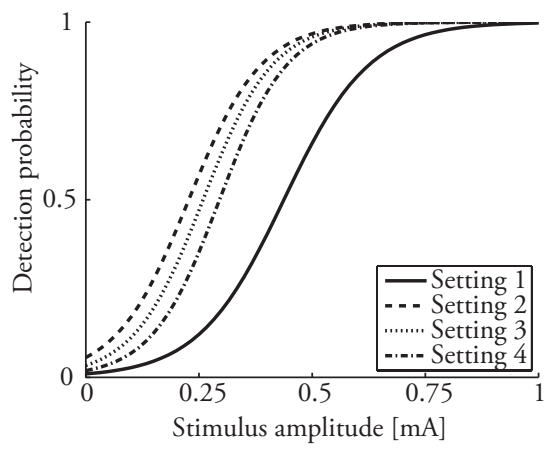

B

Figure 5-3 Psychophysical curves for experiments 1 (A) and 2 (B) for each stimulus setting (Table 5-1). The curves are obtained from the regression parameters (Table 5-3).

\section{RESULTS}

A total of 30 subjects divided over two experiments participated in the experiment. The second day data of two subjects in the first experiment, as well as data of one subject for both study days in the second experiment, were excluded from the analysis due to technical issues. About 50 stimulus response pairs $(\mathrm{SRP})(\mathrm{mean}=49.6, \mathrm{SD}=4.2)$ were available per subject, per setting, per study day. Therefore, subjects were presented with about 400 stimuli in total.

Table 5-2 presents the results of the two GLMM models. For both experiments, the intercept, stimulus amplitude, stimulation time, and the interaction between setting and stimulus amplitude as fixed effects significantly affected to the detection probability. While insignificant for the first experiment, setting had a significant effect on the detection probability in the second experiment. Moreover, the detection probability was similar on both study days.

Table 5-2 Type III Wald statistics.

\begin{tabular}{|c|c|c|c|c|}
\hline \multirow[t]{2}{*}{ Parameter } & \multicolumn{2}{|c|}{ Experiment 1} & \multicolumn{2}{|c|}{ Experiment 2} \\
\hline & $\chi^{2}(d f)$ & $p$ & $\chi^{2}(d f)$ & $p$ \\
\hline (intercept) & $78.3(1)$ & $<.001$ & $41.8(1)$ & $<.001$ \\
\hline Stimulus amplitude & $81.5(1)$ & $<.001$ & $17.9(1)$ & $<.001$ \\
\hline Setting & $1.4(3)$ & .705 & $26.7(3)$ & $<.001$ \\
\hline Stimulation time & $24.4(1)$ & $<.001$ & $34.7(1)$ & $<.001$ \\
\hline Study day & $0.2(1)$ & .640 & $0.1(1)$ & .791 \\
\hline Setting $\times$ stimulus amplitude & $106.1(3)$ & $<.001$ & $49.1(3)$ & $<.001$ \\
\hline
\end{tabular}




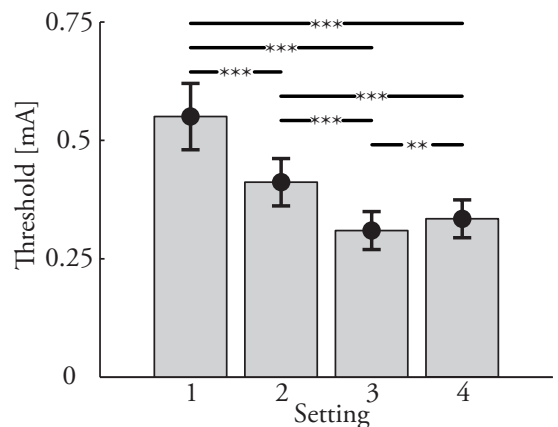

A

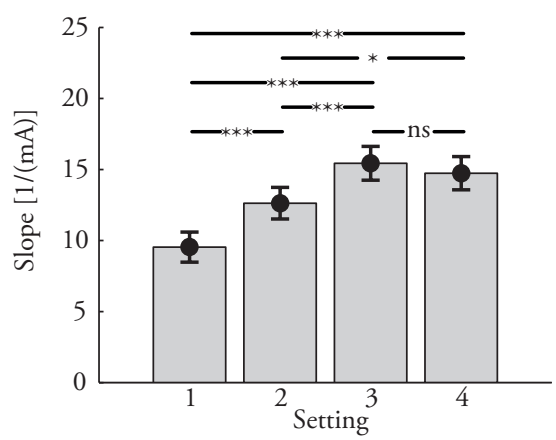

C

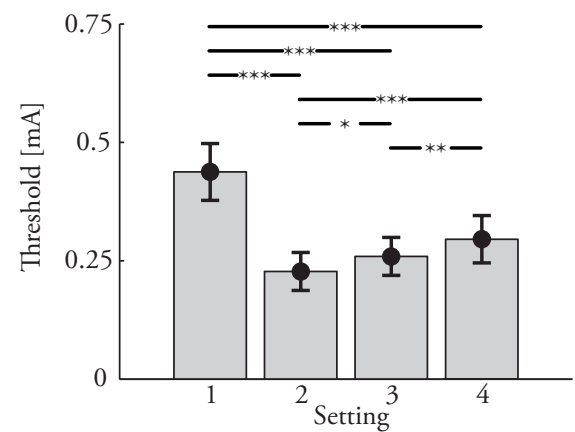

B

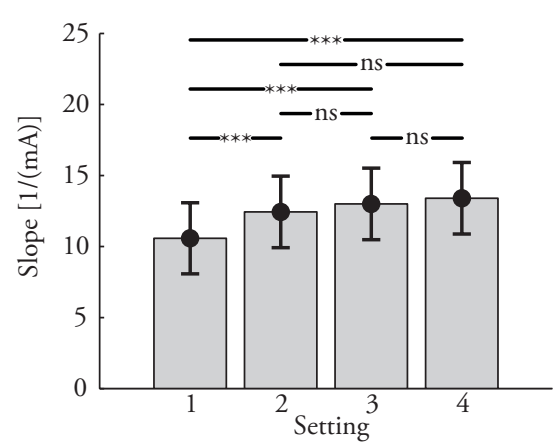

D

Figure 5-4 Estimated thresholds (A and B) and slopes (C and D) and corresponding standard errors for both experiments for each stimulus setting (Table 5-1). Overall, increasing the pulse-width and number of pulses resulted in a decrease in detection threshold and in an increase in the slope. Increasing the number of pulses results in an increased detection threshold, but no observable difference in the slope. ${ }^{*},{ }^{* *}$, and ${ }^{* *}$ indicate a significant mean difference with a value of $p<.05, p<.01$, and $p<.001$, respectively.

The estimated log-odds for the regression parameters and corresponding 95\% confidence intervals are presented in Table 5-3. Note that the stimulation time variable was transformed using a z-transformation prior to the analysis. Therefore, all obtained parameters reflect the estimated parameters at the middle of the experiment. The effect of time on the psychophysical curve ranged from 0.01 to $0.02[(\mathrm{~mA}) / \mathrm{min}]$ and was similar in both experiments. The regression parameters were inverse-logit transformed to obtain the logistic psychophysical curves for all settings at the first study day (Figure 5-3).

The estimated thresholds and slopes in both experiments are presented in Figure 5-4. Post hoc comparisons between settings (Table 5-1) showed that increasing the PW from 420 to $840 \mu$ for a single-pulse decreased the threshold and increased the slope (Figure 5-4, A and C). Increasing the NoP from 1 to 2 pulses resulted in a decrease in threshold and increase in slope as well (Figure 5-4, A - D). Moreover, increasing 
the IPI between two consecutive pulses slightly increased the threshold, but did not significantly affect the slope.

\section{DISCUSSION}

We performed two psychophysical experiments, each including 15 human subjects, to study the effect of temporal stimulus properties on the nociceptive detection probability. Subjects were presented stimuli with four different combinations of temporal properties. Temporal properties in the first experiment were chosen such that the PW, NoP, and IPI were varied. The properties in the second experiment were chosen to put more emphasize on the NoP and IPI. We studied whether the different temporal stimulus properties affect the detection probability in terms of the threshold and slope and whether differences reflect contributions of nociceptive processes.

A needle electrode (Steenbergen et al., 2012) was used for intra-epidermal electrical stimulation. Recent studies have shown that this type of stimulation device allows the preferential stimulation of nociceptive A $\delta$-fibers, provided that the amplitudes of stimuli are below twice the detection threshold (Legrain \& Mouraux, 2013; Mouraux et al., 2010). In the present experiment, stimulus amplitudes were chosen according to an adaptive stimulus selection procedure (Chapter 2 and Chapter 3) such that the amplitudes were always near the detection threshold. Some of the presented single-pulse stimuli might have had amplitudes near twice the estimated detection threshold and

Table 5-3 Regression parameter estimates of the fixed effects and corresponding confidence intervals. Presented values are the log-odds.

\begin{tabular}{|c|c|c|c|c|}
\hline \multirow[t]{2}{*}{ Parameter } & \multicolumn{2}{|c|}{ Experiment 1} & \multicolumn{2}{|c|}{ Experiment 2} \\
\hline & Estimate & $95 \%$ confidence interval & Estimate & $95 \%$ confidence interval \\
\hline (intercept) & -5.25 & {$\left[\begin{array}{ll}-6.41 & -4.09\end{array}\right]$} & -4.63 & {$\left[\begin{array}{lll}-6.03 & -3.23\end{array}\right]$} \\
\hline Stimulus amplitude & 9.54 & {$\left[\begin{array}{lll}7.47 & 11.61\end{array}\right]$} & 10.58 & {$\left[\begin{array}{lll}5.68 & 15.49\end{array}\right]$} \\
\hline \multicolumn{5}{|l|}{ Setting } \\
\hline Setting 2 & 0.05 & {$\left[\begin{array}{lll}-0.60 & 0.71\end{array}\right]$} & 1.80 & {$\left[\begin{array}{ll}1.01 & 2.60\end{array}\right]$} \\
\hline Setting 3 & 0.47 & {$\left[\begin{array}{ll}-0.38 & 1.33\end{array}\right]$} & 1.26 & {$\left[\begin{array}{lll}0.56 & 1.96\end{array}\right]$} \\
\hline Setting 4 & 0.32 & {$\left[\begin{array}{ll}-0.53 & 1.17\end{array}\right]$} & 0.67 & {$\left[\begin{array}{lll}-0.05 & 1.39\end{array}\right]$} \\
\hline Stimulation time & -0.52 & {$[-0.73-0.32]$} & -0.68 & {$\left[\begin{array}{lll}-0.91 & -0.46\end{array}\right]$} \\
\hline Study day & -0.34 & {$\left[\begin{array}{ll}-1.77 & 1.09\end{array}\right]$} & 0.12 & {$\left[\begin{array}{ll}-0.76 & 1.00\end{array}\right]$} \\
\hline \multicolumn{5}{|c|}{ Setting $\times$ stimulus amplitude } \\
\hline Setting 2 & 3.09 & {$\left[\begin{array}{ll}1.98 & 4.20\end{array}\right]$} & 1.86 & {$\left[\begin{array}{lll}0.99 & 2.72\end{array}\right]$} \\
\hline Setting 3 & 5.90 & {$[4.557 .25]$} & 2.42 & {$\left[\begin{array}{lll}1.49 & 3.36\end{array}\right]$} \\
\hline Setting 4 & 5.20 & {$[3.926 .47]$} & 2.82 & {$\left[\begin{array}{lll}1.88 & 3.75\end{array}\right]$} \\
\hline
\end{tabular}


possibly co-activated A $\beta$-fibers. Even if these activated fibers would have promoted the detection probability of these stimuli, the corresponding SRPs would have a small influence on the estimation of the detection threshold, as these SRPs were not near the detection threshold. Hence, the detection of a stimulus is likely to be preferentially mediated by activation of $A \delta$-fibers, without substantial contribution of co-activated $A \beta$-fibers. To further explore the contributions of $A \beta$-fibers, future studies could include evoked potentials to demonstrate the preferential stimulation of nociceptive fibers (e.g., see Mouraux et al. (2010), Mouraux et al. (2014), van der Heide et al. (2009)).

The detection thresholds found in this study are higher than those presented by Otsuru et al. (2010) and Mouraux et al. (2010), but similar to those previously observed in our group (Chapter 2; Steenbergen et al., 2012). The design of the electrode is the biggest difference between these studies. While Otsuru et al. (2010) and Mouraux et al. (2010) used a single-needle electrode, we used a compound electrode consisting of five needles (Figure 5-2). As the needles were interconnected, the total current is, assuming ideal needles and with similar electrical impedances, evenly distributed over the five needles. As a result, the total current necessary for neural activation is about five times higher for a five-needle electrode than for a single-needle electrode. Therefore, the thresholds presented in this paper should be divided by five for a better comparison. Doing so would show that the thresholds are then in a similar range as those presented by Otsuru et al. (2010) and Mouraux et al. (2010).

Three sets of temporal settings in the first experiment were also used in the second experiment (see Table 5-1), and thus similar results were expected. However, the estimated thresholds in the first experiment are higher than those observed in the second experiment. The difference between the two experiments was the electrode configuration. A conducting pad covering the flat electrodes was used as anode in the first experiment, but was not used in the second experiment. This slightly increased the length of the needles and could possibly decrease the distance between needles and nerve fibers. Moreover, the location of the anode in the second experiment was further away from the needles than the conducting pad was. Therefore, a difference in neuro-electrical interface was present between the first and second experiments, likely explaining the difference in thresholds for the same temporal properties. However, this does not affect the overall conclusions of this paper.

When comparing the estimated thresholds of the two single-pulse stimuli with different PW, a higher detection threshold was observed when the PW was 


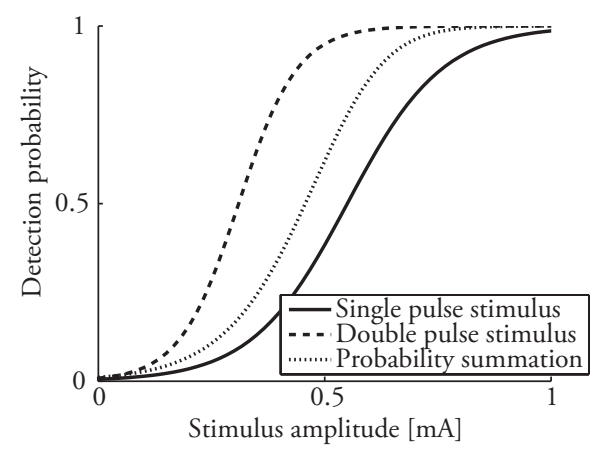

A

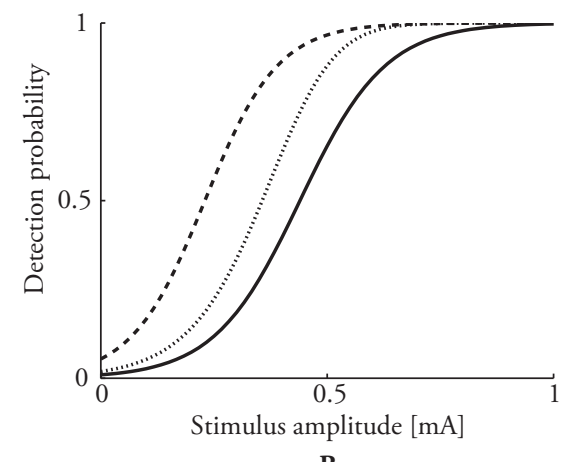

B

Figure 5-5 Effect of double-pulse stimulation. (A) presents data from the first experiment, and (B) from the second experiment. Based on probability summation, a decrease in threshold and increase in slope are expected for double-pulse stimuli. However, the estimated curves for double-pulse stimuli show even lower thresholds, and slightly steeper slopes.

$420 \mu$ s than when the PW was $840 \mu$ s. The threshold found for the shorter PW was, on average, $0.55 \mathrm{~mA}$ in the first experiment. This indicates that a total charge of about $420 \mu \mathrm{s} \times 0.55 \mathrm{~mA}=0.23 \mu \mathrm{C}$ was necessary for detection. Given a mean threshold of about $0.41 \mathrm{~mA}$ for the longer pulse stimulus, a charge of about $0.34 \mu \mathrm{C}$ was necessary for detection. This charge is about 1.5 times higher than the charge for the shorter pulse stimulus. Therefore, increasing the PW results in an increase in charge necessary to elicit a detection. This phenomenon is related to the well-known strength-duration relationship describing the minimum charge required for peripheral activation (Geddes, 2004; Rollman, 1969). Given the estimated thresholds, the chronaxie value was calculated as $435 \mu \mathrm{s}$. While chronaxie values are dependent on several external factors making estimates difficult to interpret (Geddes, 2004), the calculated value here is relatively high, supporting the argument of preferentially stimulating A $\delta$-fibers (Li \& Bak, 1976; West \& Wolstencroft, 1983).

When the number of pulses is increased in a stimulus, a decrease in threshold and an increase in slope are expected based on pure probability summation (Figure 5-1). Using the regression parameters found for the single-pulse stimulus (Table 5-3), the expected psychophysical curve for double-pulse stimuli can be calculated. Figure 5-5 shows the psychophysical curves for single-pulse stimuli, double-pulse stimuli, and the curve based on pure probability summation. In both experiments, the estimated thresholds for double-pulse stimuli are clearly lower than the expected thresholds based on pure probability summation. This suggests that pure probability summation does not fully account for a decrease in the threshold when the NoP is increased, and thus the detection probability of the second pulse is facilitated by the first pulse. Candidate 
mechanisms explaining this facilitation might reside at the peripheral as well as at the central level and both possibilities are considered below.

When considering the possibility of a peripheral facilitatory mechanism, it should be noted that a single stimulus pulse can activate a certain number of neural fibers in the skin, generating a certain amount of neural activity. The number of activated fibers and hence the amount of neural activity will increase with the pulse amplitude (and PW), and this amount determines the probability that the pulse is detected by the subject. Hence, if the detection probability of the second pulse in a double-pulse stimulus is increased by a peripheral mechanism, then the second pulse must generate a larger amount of neural activity than the first pulse, most likely by activating an additional number of fibers. As these additional fibers were not activated by the first pulse in the double-pulse stimulus, the presence of this pulse must have caused a change in the excitability of these fibers. This phenomenon is known as subthreshold superexcitability and is demonstrated in human experiments (Bostock et al., 2005; Shefner, Preston, \& Logigian, 1996). In these studies, motor axons were found to be superexcitable up to about $30 \mathrm{~ms}$ after a near-threshold depolarizing pulse. As far as we are aware, no such effects for IPIs longer than $30 \mathrm{~ms}$ were reported. Also, we did not find reports of such effects specifically for cutaneous nociceptive fibers. In our study, we observed facilitation for IPIs ranging between 10 and $100 \mathrm{~ms}$. Although this range exceeds the IPIs reported in literature, subthreshold superexcitability effects may still have occurred, as cutaneous nociceptive fibers have a much smaller diameter and might have longer time constants.

One of the possible central mechanisms leading to facilitation of the second pulse by the first is temporal summation of postsynaptic potentials. Another possibility is short-term synaptic plasticity (Zucker \& Regehr, 2002), such as paired-pulsefacilitation or augmentation, enhancing the postsynaptic response after the second pulse which follows the first pulse. This effect was observed in synapses at several locations in the nociceptive system for IPIs ranging between tens and hundreds of ms (Luo, Kuner, \& Kuner, 2014). Both temporal summation and short-term plasticity can result in facilitation of the second pulse, but no distinction between the two can be made at this point.

With the current observations, peripheral and central contributions cannot be distinguished and future studies could focus on characterizing peripheral contributions. A first step would be to study subthreshold superexcitability of nociceptive axons. Another option which helps separating peripheral from central contributions is recording the membrane potentials using microneurography. 


\section{CONCLUSION AND OUTLOOK}

In the present study, it was demonstrated that varying the temporal properties of intra-epidermal electrical stimuli result in variations in nociceptive processing. The estimated thresholds and slopes corresponding to the selection of temporal properties suggest that contributions of (a combination of) peripheral and central nociceptive mechanisms can be reflected in psychophysical functions. The stimulation paradigm has the potential of being of additional value to currently used neurophysiological and psychophysical measurements for quantification of the current state of nociceptive function. We argue that there is a need of further exploration of behavior of underlying mechanisms using electrical stimuli. Pharmacological interventions which selectively affect peripheral (e.g., application of capsaicin) and/or central mechanisms (e.g., (s)-ketamine) can be used for validation of the responsiveness of the presented methodology. Moreover, combining the presented methodology with computational models of nociceptive mechanisms has the potential of exploiting the newly gained observability the nociceptive system using a mechanism based interpretation (e.g., based on a system identification approach).

\section{REFERENCES}

Arendt-Nielsen, L., \& Yarnitsky, D. (2009). Experimental and Clinical Applications of Quantitative Sensory Testing Applied to Skin, Muscles and Viscera. Journal of Pain, 10(6), 556-572.

Backonja, M. M., Attal, N., Baron, R., Bouhassira, D., Drangholt, M., Dyck, P. J., . . Ziegler, D. (2013). Value of quantitative sensory testing in neurological and pain disorders: NeuPSIG consensus. Pain, 154(9), 1807-1819.

Bates, D., Maechler, M., Bolker, B., \& Walker, S. (2014). Ime4: Linear mixed-effects models using Eigen and S4, R package version 1.1-7.

Bostock, H., Lin, C. S. Y., Howells, J., Trevillion, L., Jankelowitz, S., \& Burke, D. (2005). After-effects of near-threshold stimulation in single human motor axons. Journal of Physiology, 564(3), 931-940.

Bromm, B., \& Lorenz, J. (1998). Neurophysiological evaluation of pain. Electroencephalography and Clinical Neurophysiology, 107(4), 227-253.

Faraggi, D., Izikson, P., \& Reiser, B. (2003). Confidence intervals for the 50 per cent response dose. Statistics in Medicine, 22(12), 1977-1988.

Geddes, L. A. (2004). Accuracy Limitations of Chronaxie Values. IEEE Transactions on Biomedical Engineering, 51(1), 176-181.

Gescheider, G. A., Berryhill, M. E., Verrillo, R. T., \& Bolanowski, S. J. (1999). Vibrotactile temporal summation: Probability summation or neural integration? Somatosensory and Motor Research, 16(3), 229-242.

Gold, J. I., \& Ding, L. (2013). How mechanisms of perceptual decision-making affect the psychometric function. Progress in Neurobiology, 103, 98-114.

Inui, K., \& Kakigi, R. (2012). Pain perception in humans: use of intraepidermal electrical stimulation. Journal of Neurology, Neurosurgery and Psychiatry, 83(5), 551-556.

Klein, S. A. (2001). Measuring, estimating, and understanding the psychometric function: a commentary. Perception and Psychophysics, 63(8), 1421-1455.

Kodaira, M., Inui, K., \& Kakigi, R. (2014). Evaluation of nociceptive A $\delta$ - and C-fiber dysfunction with lidocaine using intraepidermal electrical stimulation. Clinical Neurophysiology, 125(9), 1870-1877. 
Latremoliere, A., \& Woolf, C. J. (2009). Central Sensitization: A Generator of Pain Hypersensitivity by Central Neural Plasticity. Journal of Pain, 10(9), 895-926.

Legrain, V., \& Mouraux, A. (2013). Activating selectively and reliably nociceptive afferents with concentric electrode stimulation: Yes we can! Provided that low stimulus intensities are used! Clinical Neurophysiology, 124(2), 424.

Li, C. L., \& Bak, A. (1976). Excitability characteristics of the A- and C-fibers in a peripheral nerve. Experimental Neurology, 50(1), 67-79.

Luo, C., Kuner, T., \& Kuner, R. (2014). Synaptic plasticity in pathological pain. Trends in Neurosciences, 37(6), $343-355$.

Mendell, L. M. (2011). Computational functions of neurons and circuits signaling injury: Relationship to pain behavior. Proceedings of the National Academy of Sciences of the United States of America, 108(SUPPL. 3), 15596-15601.

Moscatelli, A., Mezzetti, M., \& Lacquaniti, F. (2012). Modeling psychophysical data at the population-level: The generalized linear mixed model. Journal of Vision, 12(11):26, 1-17.

Mouraux, A., Iannetti, G. D., \& Plaghki, L. (2010). Low intensity intra-epidermal electrical stimulation can activate A $\delta$-nociceptors selectively. Pain, 150(1), 199-207.

Mouraux, A., Marot, E., \& Legrain, V. (2014). Short trains of intra-epidermal electrical stimulation to elicit reliable behavioral and electrophysiological responses to the selective activation of nociceptors in humans. Neuroscience Letters, 561, 69-73.

Otsuru, N., Inui, K., Yamashiro, K., Miyazaki, T., Takeshima, Y., \& Kakigi, R. (2010). Assessing Adelta fiber function with lidocaine using intraepidermal electrical stimulation. Journal of Pain, 11(7), 621-627.

R Core Team. (2014). R: A Language and Environment for Statistical Computing. Vienna, Austria: R Foundation for Statistical Computing. Retrieved from http://www.R-project.org

Rolke, R., Baron, R., Maier, C., Tölle, T. R., Treede, R. D., Beyer, A., . . Wasserka, B. (2006). Quantitative sensory testing in the German Research Network on Neuropathic Pain (DFNS): Standardized protocol and reference values. Pain, 123(3), 231-243.

Rollman, G. B. (1969). Electrocutaneous stimulation: Psychometric functions and temporal integration. Perception and Psychophysics, 5(5), 289-293.

Sandkühler, J. (2009). Models and mechanisms of hyperalgesia and allodynia. Physiological Reviews, 89(2), 707-758.

Shefner, J. M., Preston, D. C., \& Logigian, E. L. (1996). Activity-dependent conduction in single motor units. Neurology, 46(5), 1387-1390.

Steenbergen, P., Buitenweg, J. R., Trojan, J., van der Heide, E. M., van den Heuvel, T., Flor, H., \& Veltink, P. H. (2012). A system for inducing concurrent tactile and nociceptive sensations at the same site using electrocutaneous stimulation. Behavior Research Methods, 44(4), 924-933.

Treede, R. D., Meyer, R. A., Raja, S. N., \& Campbell, J. N. (1992). Peripheral and central mechanisms of cutaneous hyperalgesia. Progress in Neurobiology, 38(4), 397-421.

Treutwein, B. (1995). Adaptive psychophysical procedures. Vision Research, 35(17), 2503-2522.

van der Heide, E. M., Buitenweg, J. R., Marani, E., \& Rutten, W. L. (2009). Single pulse and pulse train modulation of cutaneous electrical stimulation: a comparison of methods. Journal of Clinical Neurophysiology, 26(1), 54-60.

Weiss, G. (1901). Sur la possibilité de rendre comparables entre eux les appareils servant à l'excitation électrique. Turin: H. Loescher.

West, D. C., \& Wolstencroft, J. H. (1983). Strength-duration characteristics of myelinated and non-myelinated bulbospinal axons in the cat spinal cord. The Journal of Physiology, 337, 37-50.

Wilder-Smith, O. H., Schreyer, T., Scheffer, G. J., \& Arendt-Nielsen, L. (2010). Patients with chronic pain after abdominal surgery show less preoperative endogenous pain inhibition and more postoperative hyperalgesia: a pilot study. Journal of Pain \& Palliative Care Pharmacotherapy, 24(2), 119-128.

Woolf, C. J. (2011). Central sensitization: Implications for the diagnosis and treatment of pain. Pain, 152(SUPPL.3), S2-S15.

Zucker, R. S., \& Regehr, W. G. (2002). Short-term synaptic plasticity. Annual Review of Physiology, 64, 355-405. 



\section{Chapter 6}

Responsiveness of electrical nociceptive detection thresholds to capsaicin (8\%) induced changes in nociceptive processing

Robert J. Doll, Guido van Amerongen, Justin L. Hay, Geert J. Groeneveld, Peter H. Veltink, Jan R. Buitenweg 
Abstract Pain disorders can be initiated and maintained by malfunctioning of one or several mechanisms underlying the nociceptive function. Psychophysical procedures allow the estimation of nociceptive detection thresholds using intra-epidermal electrical stimuli. By varying the temporal properties of electrical stimuli, various contributions of nociceptive processes to stimulus processing can be observed. To observe the responsiveness of nociceptive thresholds to changes in nociceptive function, a model of capsaicin induced nerve defunctionalization was used. Its effect on nociceptive detections thresholds was investigated over a period of 84 days. A cutaneous capsaicin (8\%) patch was applied for 60 minutes to the upper leg of 8 healthy human subjects. Single and double pulse electrical stimuli were presented in a pseudo-random order using an intra-epidermal electrode. Stimuli and corresponding responses were recorded on both treated and untreated skin areas prior to capsaicin application and on days $2,7,28$, and 84 . Increases in electrical detection thresholds at the capsaicin area were observed on days 2 and 7 for single pulse stimuli. Detection thresholds corresponding to double pulse stimuli were increased on days 7 and 28, suggesting a delayed and longer lasting effect on double pulse stimuli. In the present study, it was demonstrated that the responsiveness of detection thresholds to capsaicin application depends on the temporal properties of electrical stimuli. The observation of capsaicin induced changes by estimation of detection thresholds revealed different time-patterns of contributions of peripheral and central mechanisms to stimulus processing. 


\section{INTRODUCTION}

Chronic pain disorders can be initiated and maintained by malfunctioning of one or several mechanisms underlying the nociceptive function (Mendell, 2011; Sandkühler, 2009; Woolf, 2011). Quantification of the contributions of these mechanisms could help identifying malfunctioning at a peripheral and central level. Although several methodologies exist to quantify pain processing, such as psychophysical and neurophysiological assessment of sensory function, it remains difficult to detect specific malfunctioning mechanisms. This could hamper mechanism-based treatment of (potential) pain syndromes such as small fiber neuropathy (Devigili et al., 2008), complex regional pain syndrome (Borchers \& Gershwin, 2014), or persistent post-surgical pain (Kehlet, Jensen, \& Woolf, 2006). Therefore, there is a need for methodology for improved observation of nociceptive processing.

Quantitative Sensory Testing (QST) methods allow psychophysical assessments of sensory function (Arendt-Nielsen \& Yarnitsky, 2009). These methods include application of a broad range of stimulus types such as thermal, mechanical, or electrical and recording corresponding responses. Particularly, thermal and electrical stimuli can be used for preferential stimulation of nociceptive nerve fibers (Inui \& Kakigi, 2012; Kodaira, Inui, \& Kakigi, 2014; Mouraux, Iannetti, \& Plaghki, 2010). An advantage of electrical stimulation is the accurate control of stimulation timing, allowing well-defined stimuli with temporal resolutions in the order of tens of $\mu$. Varying the temporal properties of rectangular-wave stimuli, such as the pulse-width (PW), number of pulses (NoP), and inter-pulse interval (IPI), allows probing of phenomena such as the strength-duration relationship (Rollman, 1969; Weiss, 1901) or temporal summation of post-synaptic activity (Mouraux, Marot, \& Legrain, 2014; van der Heide, Buitenweg, Marani, \& Rutten, 2009).

Within a single experiment, simultaneous recording of responses to stimuli with different temporal properties could help observe the mechanisms involved in the nociceptive system in more detail. The feasibility of using sequences of stimuli with multiple temporal properties for simultaneously obtaining information about nociceptive stimulus processing was demonstrated in healthy human subjects within a single ten-minute experiment (Chapter 5). Stimuli with different temporal properties were presented in a pseudo-random order and psychophysical curves were estimated per stimulus type. Differences in curves between single pulse stimuli with varying PWs were related to the strength-duration curve and reflected peripheral mechanisms of nociceptive processing. The difference between the curve of a single pulse stimulus and 
a double pulse stimulus demonstrated facilitation of the detection probability of the second pulse induced by the first pulse.

Observation of pharmacologically-induced changes in nociceptive function in healthy human subjects is a next step in identifying the usability of intra-epidermal electrical stimulation. A good candidate for inducing temporary changes is the application of a cutaneous patch containing an 8\%-dose of capsaicin. It was shown that the application of capsaicin results in temporary nerve defunctionalization by retraction of A $\delta$-fibers and C-fibers (Anand \& Bley, 2011; Kennedy et al., 2010; Polydefkis et al., 2004). Skin biopsies show that the intra-epidermal nerve fiber density (IENFD) is reduced after capsaicin application and shows a return to baseline within 6 months (Kennedy et al., 2010; Polydefkis et al., 2004). As a result, nociceptive, but also tactile, thresholds are temporarily increased for up to a week after application (Kennedy et al., 2010; Mouraux et al., 2010; Ragé et al., 2010). Moreover, temporary sensitization occurs at both a peripheral and central level right after capsaicin application (Sandkühler, 2009; Woolf, 2011).

In this study, a single application of an 8\%-dose capsaicin patch was used to induce changes in nociceptive function in healthy human subjects. The main objective was to simultaneously observe the responsiveness of multiple nociceptive thresholds to changes in nociceptive function over a time period of 84 days after capsaicin application. The nociceptive function was psychophysically probed in a simple detection task using intra-epidermal electrical stimulation with a variety in temporal electrical stimulus properties. Series of stimulus-response pairs were recorded prior to capsaicin application and on days $2,7,28$, and 84 on both treated and untreated skin areas.

\section{METHODS}

\section{Subjects}

After approval of the LUMC Ethics Committee and in accordance with the declaration of Helsinki, 12 healthy subjects (6 men, 6 women) were enrolled after providing written informed consent. Four subjects were not included due to issues with the needle electrode at the start of this study, leaving 8 subjects ( 5 men, 3 women; mean age $=22.5, \mathrm{SD}=2.0$ ). Inclusion criteria were: $18-65$ years old and a body mass index between $18-30 \mathrm{~kg} \cdot \mathrm{m}^{-2}$, good medical condition defined as absence of clinically significant findings in their medical history, physical examination and vital signs. Exclusion criteria were pregnancy, dark skin (Fitzpatrick scale type V or VI), illicit drug use, frequent caffeine use ( $>8$ units/day), smokers ( $>5$ cigarettes/day), extreme responders to 
capsaicin $0.075 \%$ cream, skin abnormalities and abnormal ECG or blood pressure. No over-the-counter medication within 3 days of nociceptive measurements was allowed. During the study, subjects were asked to refrain from strenuous physical exercise, use of all (methyl)xanthenes, and alcohol. Female subjects attended study-day 0 while in the follicular phase.

\section{Experiment design}

Subjects visited the lab on 5 days during a period of 84 days. Two adjacent areas were marked on subjects' distal lateral thigh non-dominant leg using transparencies. A cutaneous $6 \times 6 \mathrm{~cm}$ patch containing $8 \% \mathrm{w} / \mathrm{w}(640 \mu \mathrm{g} / \mathrm{cm} 2)$ capsaicin (Qutenza, Astellas Pharma B.V., Leiden, the Netherlands) was applied about $10 \mathrm{~cm}$ proximal to the knee for 60 minutes. The adjacent untreated area was proximal to the capsaicin treated area and served as the control. A 60 minute pre-treatment with EMLA 5\% was applied before capsaicin application. Psychophysical recordings took place prior to capsaicin application (D0), and on days 2, 7, 28, and 84 on both the treated and untreated areas.

\section{Test-stimuli}

An electrode consisting of an array of five interconnected needles and four interconnected flat electrodes (Steenbergen et al., 2012) was attached to either the treated or untreated skin area. The needles served as cathode and a conducting pad covering the flat electrodes as anode (Steenbergen et al., 2012). Each visit, the skin area to which the electrode was attached first was randomly determined.

Cathodic rectangular pulses were applied as test-stimuli using four different combinations of temporal properties (Table 6-1). Stimulus amplitudes were selected according to an adaptive probing procedure (Chapter 2). The procedure started with a pre-defined set of 5 equidistant stimulus amplitudes between 0 and $0.4 \mathrm{~mA}$ for single pulse stimuli and between 0 and $0.2 \mathrm{~mA}$ for double pulse stimuli. The amplitude of the upcoming stimulus was randomly selected from this set. All amplitudes in the set were increased and decrease with a fixed step-size after a not-detected stimulus and detected

Table 6-1 Temporal stimulus properties: pulse-width (PW), number of pulses (NoP), and inter-pulse interval (IPI).

\begin{tabular}{llll}
\hline & PW $[\mu \mathrm{s}]$ & NoP & IPI $[\mathrm{ms}]$ \\
\hline Setting 1 & 210 & 1 & - \\
Setting 2 & 525 & 1 & - \\
Setting 3 & 525 & 2 & 20 \\
Setting 4 & 525 & 2 & 50 \\
\hline
\end{tabular}


stimulus, respectively. The step-size was $0.1 \mathrm{~mA}$ for single pulse stimuli, and $0.05 \mathrm{~mA}$ for double pulse stimuli. The different stimulus settings were presented in a randomly intermingled sequence.

Subjects were instructed to indicate whether they detected a stimulus by releasing a response button and to press the button again after about a second. Due to this, the inter-stimulus interval after a detected stimulus was longer than after a not-detected stimulus. Besides the fixed inter-stimulus interval, an extra random interval between 0.6 and 1 second was added to reduce the stimulation predictability. This resulted in a mean inter-stimulus interval of 2.7 seconds and 3.7 seconds after a not-detected stimulus and after a detected stimulus, respectively. A custom computer program (written in LabVIEW 2011, SP1) controlled all stimulation procedures, as well as the registration of stimulus amplitudes in $\mathrm{mA}$, stimulation times in milliseconds, and responses to stimuli (i.e., detected or not-detected).

\section{Statistical Analysis}

All data preparation was performed in MATLAB 8.1 (MathWorks, Inc, Natick, Massachusetts, U.S.A.). Statistical modelling was performed using the lme4 library (Bates, Maechler, Bolker, \& Walker, 2014) in the R software package (R Core Team, 2014).

Two types of generalized linear mixed models (GLMM) using a logit link function were built to estimate the detection probability given the stimulus amplitude. The first type of model was to observe the effect of temporal stimulus properties on the detection probability and therefore only included the D0 data obtained at the untreated skin area. The second type of model was used to observe the effect of capsaicin application on the detection probability. A model of the second type was built per setting.

For the first model, the intercept, stimulus amplitude $[\mathrm{mA}]$, setting, stimulation time [s], and the interaction between the stimulus amplitude and setting were included as fixed effects. Between subjects random effects were included for the intercept, stimulus amplitude, and setting. For the second model, the intercept, stimulus amplitude, study day, location, stimulation time, and the interaction between study day and location were included as fixed effects. Between subjects random effects were included for the intercept, stimulus amplitude, study day, and location. An unstructured covariance matrix was used to model the random effects for both model types. 


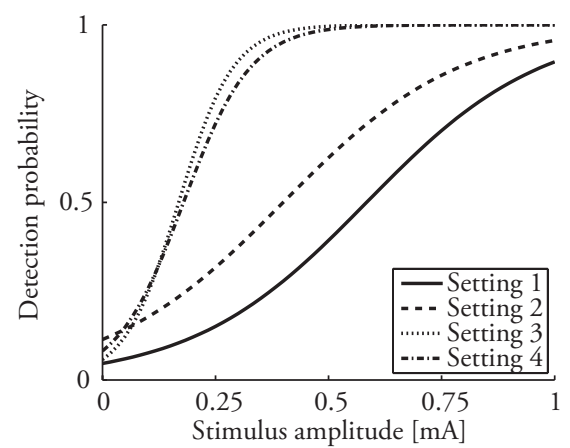

Figure 6-1 Psychophysical curves for each stimulus setting on the untreated skin area prior to capsaicin application (Table 6-1). The curves are obtained from the regression parameters (Table 6-3).

For both types of models, the stimulation time variable was centered and scaled prior to analysis to speed up the estimation process. Type III Wald $\chi^{2}$ statistics were used to test the main and interaction effects of the fixed effects. Confidence intervals of the regression parameters were based on Wald-z statistics. Threshold estimates were obtained from the regression parameters and corresponding standard errors were approximated using the Delta procedure (Faraggi, Izikson, \& Reiser, 2003; Moscatelli, Mezzetti, \& Lacquaniti, 2012). Post-hoc comparisons were performed using Bonferroni p-value correction. For the first model type, thresholds and slopes between settings were compared. For the second model type, the thresholds between stimulation locations were compared on each study day.

\section{RESULTS}

All 8 subjects completed the experiment. Out of a total of 14,857 stimuli, 843 stimuli were excluded for analysis due to technical issues. About 46 stimuli and corresponding responses $($ mean $=46.4, \mathrm{SD}=3.3)$ were available per subject, per study day, per skin area, per setting. Therefore, subjects were presented with about 1,840 stimuli in total.

Table 6-2 Comparison between temporal stimulus properties: type III Wald statistics.

\begin{tabular}{lrr}
\hline Parameter & \multicolumn{1}{c}{$\chi^{2}(d f)$} & \multicolumn{1}{c}{$p$} \\
\hline (intercept) & $12.1(1)$ & $<.001$ \\
Stimulus amplitude & $3.3(1)$ & .070 \\
Setting & $6.5(3)$ & .090 \\
Time & $1.7(1)$ & .186 \\
Stimulus amplitude $\times$ setting & $53.2(3)$ & $<.001$ \\
\hline
\end{tabular}




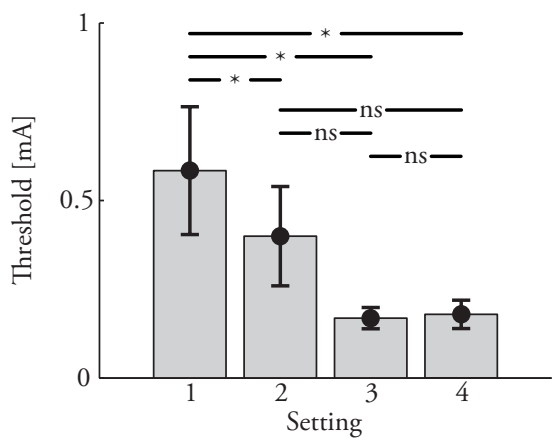

A

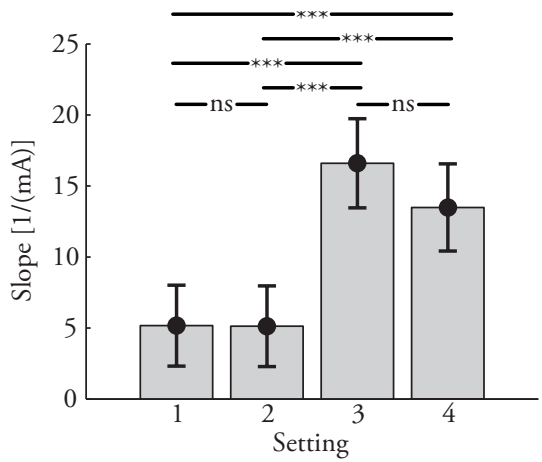

B

Figure 6-2 Estimated thresholds (A) and slopes (B) and corresponding standard errors for each stimulus setting (Table $6-1){ }^{*},{ }^{* *}$, and ${ }^{* * *}$ indicate a significant mean difference with a value of $p<.05, p<.01$, and $p<.001$, respectively.

\section{Effect of temporal stimulus parameters}

Table 6-2 presents the results of the GLMM analyses. Only the intercept and the interaction between the stimulus amplitude and setting significantly affected the detection probability. The stimulus amplitude, setting, and stimulation time did not affect the detection probability.

The estimated log-odds and corresponding $95 \%$ confidence intervals are presented in Table 6-3. Note that the stimulation time variable was transformed using a z-transform prior to the analysis. Therefore, all obtained parameters reflect the estimated parameters at the middle of the experiment. The regression parameters are inverse-logit transformed to obtain the psychophysical curves for all settings (Figure 6-1).

Table 6-3 Comparison between temporal stimulus properties: regression parameter estimates of the fixed effects and corresponding confidence intervals. Presented values are the log-odds.

\begin{tabular}{|c|c|c|c|}
\hline \multicolumn{2}{|l|}{ Parameter } & Estimate (SE) & $95 \%$ conf. int. \\
\hline \multicolumn{2}{|l|}{ (intercept) } & $-3.02(0.87)$ & {$[-4.71-1.32]$} \\
\hline \multicolumn{2}{|l|}{ Stimulus amplitude } & $5.17(2.85)$ & {$\left[\begin{array}{ll}-0.41 & 10.75\end{array}\right]$} \\
\hline \multicolumn{4}{|l|}{ Setting } \\
\hline & Setting 2 & $0.97(0.54)$ & {$\left[\begin{array}{lll}-0.10 & 2.03\end{array}\right]$} \\
\hline & Setting 3 & $0.22(0.86)$ & {$\left[\begin{array}{lll}-1.46 & 1.89\end{array}\right]$} \\
\hline & Setting 4 & $0.60(0.84)$ & {$\left[\begin{array}{lll}-1.06 & 2.25\end{array}\right]$} \\
\hline \multicolumn{2}{|l|}{ Stimulation time } & $-0.10(0.08)$ & {$\left[\begin{array}{lll}-0.26 & 0.05\end{array}\right]$} \\
\hline \multicolumn{4}{|c|}{ Stimulus amplitude $\times$ setting } \\
\hline \multicolumn{2}{|c|}{ Amplitude $\times$ Setting 2} & $-0.04(0.68)$ & {$\left[\begin{array}{lll}-1.36 & 1.29\end{array}\right]$} \\
\hline \multicolumn{2}{|c|}{ Amplitude $\times$ Setting 3} & $11.43(1.71)$ & {$\left[\begin{array}{lll}8.09 & 14.77\end{array}\right]$} \\
\hline \multicolumn{2}{|c|}{ Amplitude $\times$ Setting 4} & $8.32(1.59)$ & {$\left[\begin{array}{lll}5.20 & 11.44\end{array}\right]$} \\
\hline
\end{tabular}


The estimated thresholds and slopes are presented in Figure 6-2. Post-hoc comparisons between settings showed that a decrease in threshold was observed when increasing the PW from 210 to $525 \mu$ s, and when increasing the PW and NoP from a single $210 \mu \mathrm{s}$ pulse to a double $525 \mu$ s pulse. No differences were observed when comparing the threshold for the single $525 \mu$ s pulse stimulus with the double $525 \mu$ s pulse stimulus. Differences between slopes were observed when increasing the NoP, but not when increasing the PW or IPI.

\section{Effect of capsaicin}

Table 6-4 presents the results of the GLMM analyses. For all four settings, the intercept, stimulus amplitude, stimulation time, and the interaction between study day and location significantly affected the detection probability. Moreover, the detection probability was not affected by study day and location in all four settings.

The estimated log-odds for the regression parameters and corresponding 95\% confidence intervals are presented in Table 6-5. Note that the stimulation time variable was transformed using a z-transform prior to the analysis. Therefore, all obtained estimates reflect the parameters at the middle of the experiment. The estimated thresholds and corresponding standard errors of all settings and days are presented in Figure 6-3. When comparing the thresholds between skin areas on the same study day, an increase in threshold was observed for single pulse stimuli (i.e., setting 1 and setting 2) on days 2 and 7. An increase in threshold was observed on days 7 and 28 for double pulse stimuli.

\section{DISCUSSION}

In this study, a single application of an $8 \%$ dose capsaicin patch was used to induce changes in nociceptive function in healthy human subjects. The main objective was to simultaneously observe the responsiveness of multiple nociceptive thresholds to changes in nociceptive function over a time period of 84 days after capsaicin application.

Table 6-4 Effect of capsaicin: type III Wald statistics.

\begin{tabular}{|c|c|c|c|c|c|c|c|c|}
\hline \multirow[t]{2}{*}{ Parameter } & \multicolumn{2}{|l|}{ Setting 1} & \multicolumn{2}{|l|}{ Setting 2} & \multicolumn{2}{|l|}{ Setting 3} & \multicolumn{2}{|l|}{ Setting 4} \\
\hline & $\chi^{2}(d f)$ & $p$ & $\chi^{2}(d f)$ & $p$ & $\chi^{2}(d f)$ & $p$ & $\chi^{2}(d f)$ & $p$ \\
\hline (intercept) & $26.8(1)$ & $<.001$ & $38.1(1)$ & $<.001$ & $56.9(1)$ & $<.001$ & $42.8(1)$ & $<.001$ \\
\hline Stimulus amplitude & $20.3(1)$ & $<.001$ & $34.2(1)$ & $<.001$ & $45.5(1)$ & $<.001$ & $30.3(1)$ & $<.001$ \\
\hline Study day & $3.0(4)$ & .565 & $7.8(4)$ & .101 & $6.5(4)$ & .162 & $0.6(4)$ & .965 \\
\hline Location & $0.4(1)$ & .551 & $2.3(1)$ & .132 & $0.8(1)$ & .380 & $0.8(1)$ & .364 \\
\hline Stimulation time & $10.4(1)$ & .001 & $39.1(1)$ & $<.001$ & $36.1(1)$ & $<.001$ & $23.1(1)$ & $<.001$ \\
\hline Day $\times$ location & $47.4(4)$ & $<.001$ & $72.8(4)$ & $<.001$ & $64.5(4)$ & $<.001$ & $69.6(4)$ & $<.001$ \\
\hline
\end{tabular}




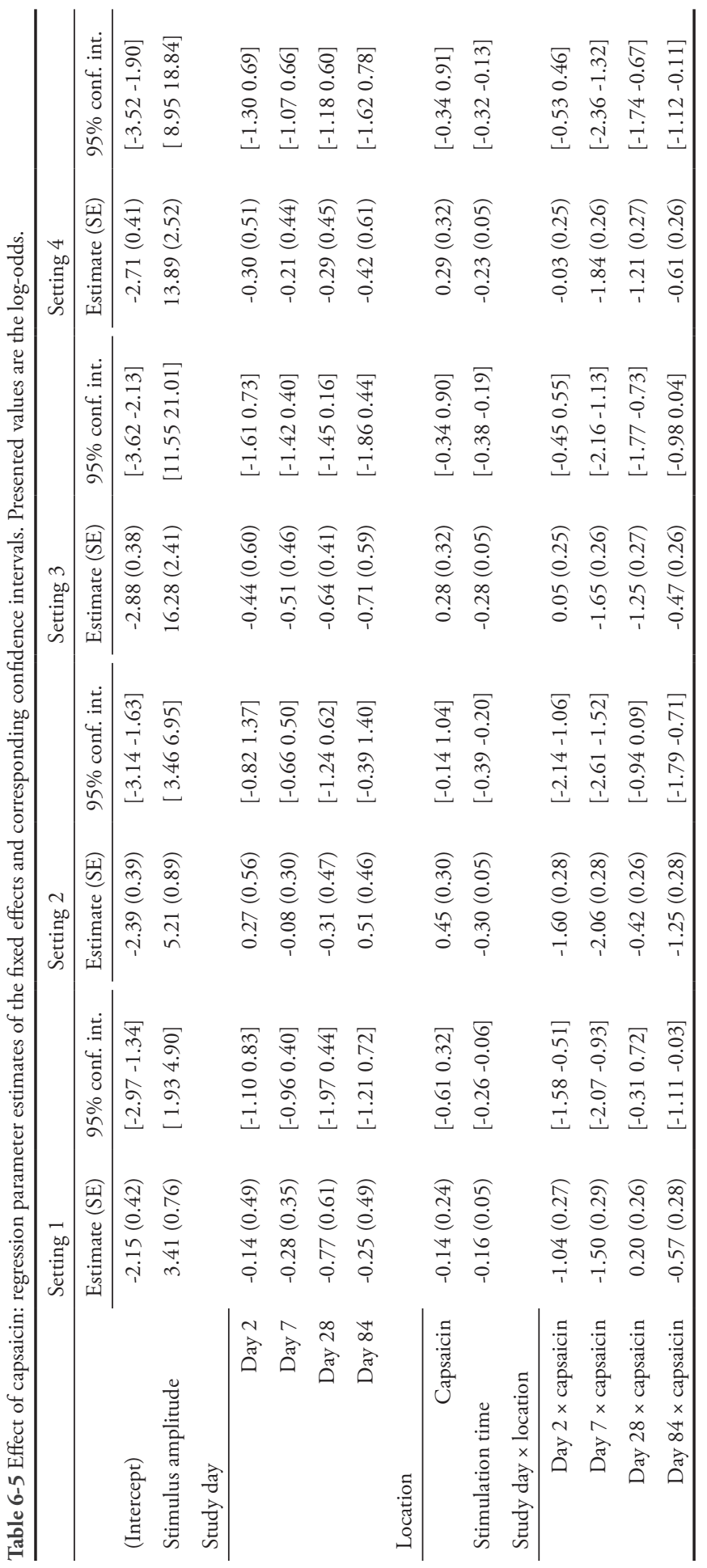



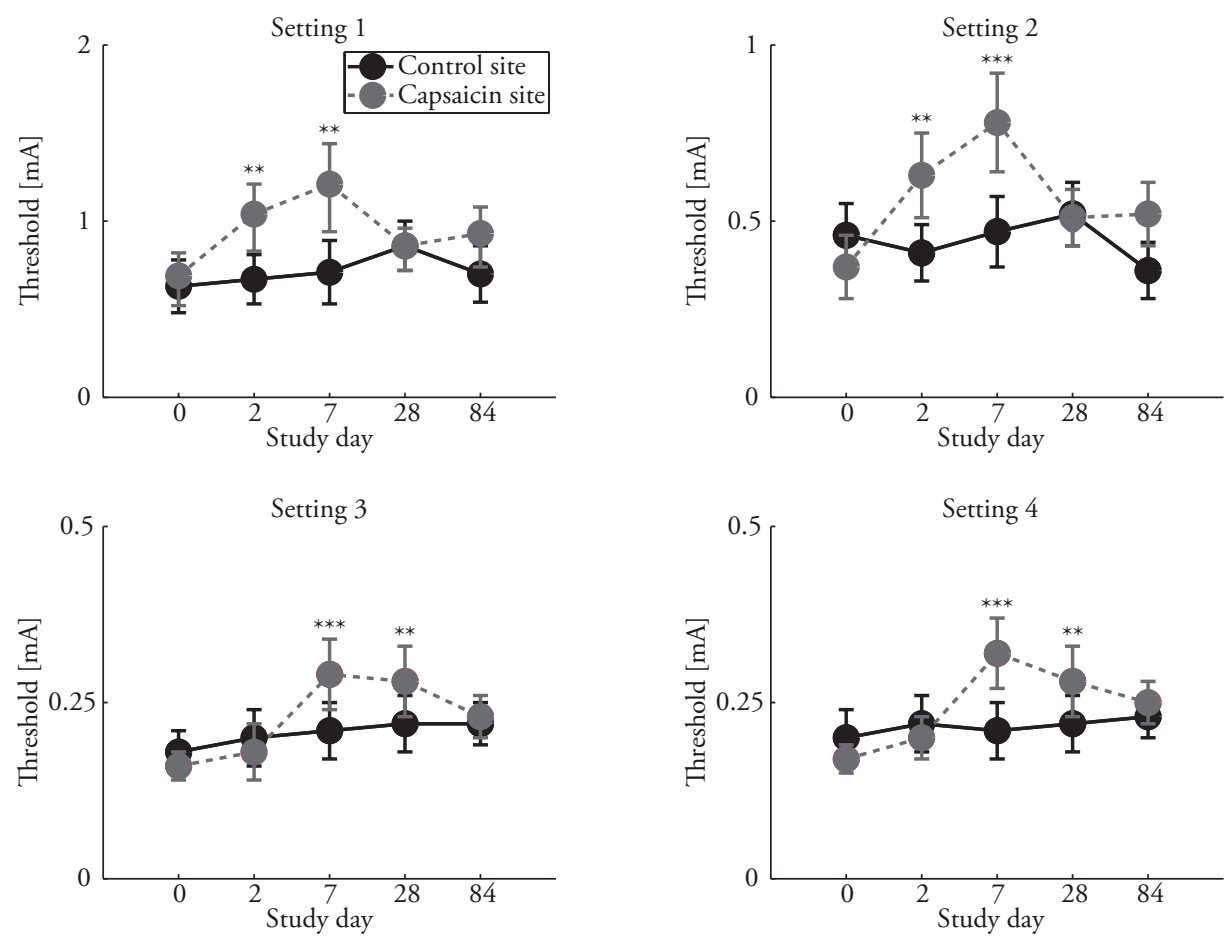

Figure 6-3 Estimated thresholds and corresponding standard errors for all four settings (Table 6-1). *, **, and *** indicate a significant mean difference between the thresholds obtained at the capsaicin treated skin area and untreated area with a value of $p<.05, p<.01$, and $p<.001$, respectively. Note the difference in $\mathrm{y}$-axis in the four sub-figures.

Nociceptive function was psychophysically probed in a simple detection task using intra-epidermal electrical stimulation with a variety in temporal electrical stimulus properties. Series of stimulus-response pairs were recorded prior to capsaicin application and on days $2,7,28$, and 84 on both treated and untreated skin areas.

A needle electrode was used for intra-epidermal electrical stimulation. Provided that the stimulus amplitudes are below twice the detection threshold, this method allowed preferential stimulation of A $\delta$-fibers (Legrain \& Mouraux, 2013; Mouraux et al., 2010). In the present experiment, stimulus amplitudes were chosen according to an adaptive stimulus selection procedure (Chapter 2 and Chapter 3 ) such that the amplitudes were always near the detection threshold. Therefore, the contributions of tactile A $\beta$-fibers to the threshold is negligible (see Chapter 5 for a comprehensive explanation).

Throughout the experiment, stimuli with four different temporal properties (Table 6-1) were presented to subjects in a pseudo-random order. The differences between the PW, NoP, and IPI of these stimuli allowed simultaneous observation of 
various contributions of nociceptive processes to stimulus processing (Chapter 5). Differences in detection probabilities of stimuli with varying PWs provide information about strength-duration properties. Differences caused by an increase in the NoP (e.g., increasing the NoP from a single pulse stimulus to a double pulse stimulus) may inform about peripheral and/or central facilitation or inhibition. The IPI then, while effects are relatively small (Chapter 5 ), might provide information regarding the time-constants of facilitation or habituation.

For the comparison between the detection probability of a single pulse $(\mathrm{NoP}=1)$ and double pulse stimulus $(\mathrm{NoP}=2)$, it should be noted that the detection probability of a double pulse stimulus, $p_{\mathrm{d}}$, depends on the detection probabilities of each of the individual pulses, $p_{s 1}$ and $p_{s 2}$, according to probability summation: $p_{d}=\left(1-p_{s 1}\right)\left(1-p_{s 2}\right)$. If the separate detection probabilities of both pulses are independent and equal, i.e., $p_{s 1}=p_{s 2}$, we refer to this as to pure probability summation, i.e., $p_{d, \text { pure }}=1-\left(1-p_{s 1}\right)^{2}$. Based on pure probability summation, the detection threshold of a double pulse stimulus is equal to the amplitude resulting in a .29 detection probability of a single stimulus. When, at a certain stimulation amplitude, the observed detection probability of a double pulse stimulus is lower or higher than expected by pure probability summation (i.e., $p_{d}<p_{d \text {, pure }}$ or $p_{d}>p_{d \text {, pure }}$ ), this indicates that the detection of the second pulse is inhibited or facilitated, respectively, by the presence of the first pulse. This effect was ascribed to either peripheral or central facilitation (Chapter 5). Peripheral facilitation might be induced due to subthreshold superexcitable properties of fibers (Bostock et al., 2005) and central facilitation due to temporal summation of post synaptic potentials or short term plasticity (Zucker \& Regehr, 2002).

\section{Effect of temporal stimulus properties}

When considering only the data obtained on the control location on day 0 (i.e., prior to capsaicin application), a decrease in threshold was observed when increasing the PW of a single pulse stimulus from $210 \mu$ s to $525 \mu$ s (Figure 6-2A). This effect of the PW is governed by the strength-duration curve (Geddes, 2004; Rollman, 1969) reflecting peripheral mechanisms of nociceptive processing and is similar to previous findings (Chapter 5).

Although a tendency towards a lower threshold for double pulse stimuli than for single pulse stimuli with the same PW (Figure 6-2A), no significant difference was observed. The slope, however, was significantly steeper for double pulse stimuli than for single pulse stimuli (Figure 6-2B). Moreover, no difference in threshold and slope 
was observed for double pulse stimuli with different IPI values. In previous studies, a difference between thresholds of single and double pulse stimuli was observed (Chapter 5). Moreover, it was also demonstrated that the detection probability of the second pulse was facilitated. In that study, however, more subjects were included and more SRPs were available for the estimation process. Therefore, we conclude that future studies should include more data if the effect of temporal stimulus properties on both the threshold and slope is of interest.

No differences in thresholds and slopes were observed for double pulse stimuli when the IPI value was increased from 20 to $50 \mathrm{~ms}$. In a previous study, it was already demonstrated that IPI has a relatively small effect on the detection probability. Only a small change in threshold was observed when increasing the IPI value from 10 to 100 ms. As mentioned above, more subjects and SRPs were available for the estimation of the detection probability. Additionally, the range of IPI values was broader than the range used in the present study. Therefore, the IPI range should be increased, as well as the number of SRPs when the effect of IPI is of interest.

\section{Effect of capsaicin}

The two adjacent stimulation locations were close together, possibly affecting the quality of the control recordings. However, the detection thresholds on the control location remained relatively constant over the study period, regardless of the setting (Table 6-4 and Table 6-5). Moreover, as the distance between the capsaicin patch and control location was relatively large $(>2 \mathrm{~cm})$, capsaicin diffusion towards the control skin area is unlikely (Selim et al., 2010). Therefore, it is unlikely that the capsaicin diffused into the control skin area and induced peripheral or central changes.

The thresholds recorded on the treated location were affected by the capsaicin application and showed increases lasting for several days. The time profiles of single pulse thresholds with different PWs were similar: thresholds were increased on days 2 and 7 and returned to baseline value within 28 days. The time profiles of double pulse stimuli with varying IPI values were also similar, but different than the profile of single pulse stimuli: thresholds were increased on days 7 and 28 , and returned to baseline value within 84 days. The different time profiles of single and double pulse thresholds suggest that various nociceptive processes are affected by capsaicin application and that these changes might be observable in psychophysical thresholds. Possible explanations for the differences in time patterns are discussed in the paragraphs below. 
Studies have shown a reduction in IENFD within a week with similar capsaicin patches (Kennedy et al., 2010; Knolle et al., 2013; Malmberg et al., 2004). It is likely that the IENFD was already reduced on day 2 as studies using a lower capsaicin dose found reductions within 2 days (Polydefkis et al., 2004). The reduction in IENFD indicates a retraction of nerve fibers (O'Neill et al., 2012) and thereby increasing the distance between the electrode surface and nerve fibers. As a result, higher stimulation currents are required to reach and activate the retracted nerve fibers. Therefore, an increase in detection thresholds can be expected when the IENFD is reduced and likely explains the increase in threshold for single pulse stimuli.

As the detection thresholds for single pulse stimuli were increased on day 2, an increase in threshold was expected for double pulse stimuli as well. Based on pure probability summation, the expected detection threshold for double pulse stimuli is equal to the amplitude resulting in a detection probability of .29 for a single pulse stimulus. With an increase in single pulse threshold, a slight decrease in slope could be expected as well, resulting in a less effective increase in double pulse threshold. However, the thresholds for double pulse stimuli were not increased on day 2. This suggests an increased facilitation on the detection probability of the second pulse in comparison with day 0 . This increased facilitation was no longer observed on day 7 , as an increase in thresholds for double pulse stimuli were observed on that day. Whether the lower threshold for double pulse stimuli can be explained by increased peripheral activity due to, for example, increased subthreshold superexcitability (Bostock et al., 2005), increased central activity (e.g., due to increased temporal summation of post synaptic potentials or short term plasticity (Zucker \& Regehr, 2002)) or a combination of the two is unclear at this point. Further research is required to be able to distinguish peripheral from central contributions.

While the detection thresholds for single pulse stimuli returned to baseline value within 28 days, the thresholds for double pulse stimuli were still increased on day 28. As the detection probability of the first pulse of a double pulse stimulus is equal to the detection probability of a single pulse stimulus, the detection probability of the second pulse must be inhibited or less facilitated. Again, this inhibition or decreased facilitation could be caused by peripheral mechanisms, by central mechanisms, or by a combination of both. Estimating slopes of psychophysical curves per study day and per stimulation location could aid in deciding whether inhibition or decreased facilitation occurs on day 28. However, due to variability, we were unable to obtain reliable slope estimates and are therefore unable to verify a change in slope. As estimation of the threshold is relatively 
simple in contrast to estimation of the slope (King-Smith \& Rose, 1997; Kontsevich \& Tyler, 1999), future studies focusing on the slope could either increase the number of stimulus-response pairs, or include more subjects.

\section{CONCLUSION AND OUTLOOK}

In the present study, it was demonstrated that the responsiveness of detection thresholds to capsaicin-induced changes in nociceptive processing depends on the temporal properties of electrical stimuli. The detection thresholds to single pulse stimuli were increased on days 2 and 7 after capsaicin application while the detection thresholds to double pulse stimuli were increased on days 7 and 28 . Overall, we demonstrated that the use of intra-epidermal electrical stimulation can be used to explore changes in nociceptive processing. A better understanding of nociceptive processing in healthy controls can be achieved by computational models based on the underlying neurophysiology. A next step for further exploration of intra-epidermal stimulation as a method to observe contributions of nociceptive mechanisms to stimulus processing is to incorporate the methods presented in this paper in a clinical setting. A first group of patients could include those scheduled for surgery. The incidence of persisting pain development after surgery is high (Perkins \& Kehlet, 2000), while treatment of settled persistent pain is relatively ineffective (Apfelbaum, Chen, Mehta, \& Gan, 2003). Following nociceptive changes prior and post-surgery using intra-epidermal electrical stimulation could be of additional value to existing QST measures used for clinical purposes (Backonja et al., 2013) in describing the state of the nociceptive system.

\section{ACKNOWLEDGEMENTS}

Partial financial support for report writing was provided by Astellas Pharma B.V., Leiden, the Netherlands.

\section{REFERENCES}

Anand, P., \& Bley, K. (2011). Topical capsaicin for pain management: Therapeutic potential and mechanisms of action of the new high-concentration capsaicin 8 patch. British Journal of Anaesthesia, 107(4), 490-502.

Apfelbaum, J. L., Chen, C., Mehta, S. S., \& Gan, T. J. (2003). Postoperative pain experience: Results from a national survey suggest postoperative pain continues to be undermanaged. Anesthesia and Analgesia, 97(2), 534-540.

Arendt-Nielsen, L., \& Yarnitsky, D. (2009). Experimental and Clinical Applications of Quantitative Sensory Testing Applied to Skin, Muscles and Viscera. Journal of Pain, 10(6), 556-572.

Backonja, M. M., Attal, N., Baron, R., Bouhassira, D., Drangholt, M., Dyck, P. J., . . Ziegler, D. (2013). Value of quantitative sensory testing in neurological and pain disorders: NeuPSIG consensus. Pain, 154(9), 1807-1819.

Bates, D., Maechler, M., Bolker, B., \& Walker, S. (2014). Ime4: Linear mixed-effects models using Eigen and S4, R package version 1.1-7. 
Borchers, A. T., \& Gershwin, M. E. (2014). Complex regional pain syndrome: A comprehensive and critical review. Autoimmunity Reviews, 13(3), 242-265.

Bostock, H., Lin, C. S. Y., Howells, J., Trevillion, L., Jankelowitz, S., \& Burke, D. (2005). After-effects of near-threshold stimulation in single human motor axons. Journal of Physiology, 564(3), 931-940.

Devigili, G., Tugnoli, V., Penza, P., Camozzi, F., Lombardi, R., Melli, G., . . Lauria, G. (2008). The diagnostic criteria for small fibre neuropathy: From symptoms to neuropathology. Brain, 131(7), 1912-1925.

Faraggi, D., Izikson, P., \& Reiser, B. (2003). Confidence intervals for the 50 per cent response dose. Statistics in Medicine, 22(12), 1977-1988.

Geddes, L. A. (2004). Accuracy Limitations of Chronaxie Values. IEEE Transactions on Biomedical Engineering, 51(1), $176-181$.

Inui, K., \& Kakigi, R. (2012). Pain perception in humans: use of intraepidermal electrical stimulation. Journal of Neurology, Neurosurgery and Psychiatry, 83(5), 551-556.

Kehlet, H., Jensen, T. S., \& Woolf, C. J. (2006). Persistent postsurgical pain: risk factors and prevention. Lancet, 367(9522), 1618-1625.

Kennedy, W. R., Vanhove, G. F., Lu, S.-p., Tobias, J., Bley, K. R., Walk, D., . . Selim, M. M. (2010). A Randomized, Controlled, Open-Label Study of the Long-Term Effects of NGX-4010, a High-Concentration Capsaicin Patch, on Epidermal Nerve Fiber Density and Sensory Function in Healthy Volunteers. The Journal of Pain, 11(6), 579-587.

King-Smith, P. E., \& Rose, D. (1997). Principles of an adaptive method for measuring the slope of the psychometric function. Vision Research, 37(12), 1595-1604.

Knolle, E., Zadrazil, M., Kovacs, G. G., Medwed, S., Scharbert, G., \& Schemper, M. (2013). Comparison of cooling and EMLA to reduce the burning pain during capsaicin $8 \%$ patch application: A randomized, double-blind, placebocontrolled study. Pain, 154(12), 2729-2736.

Kodaira, M., Inui, K., \& Kakigi, R. (2014). Evaluation of nociceptive A $\delta$ - and C-fiber dysfunction with lidocaine using intraepidermal electrical stimulation. Clinical Neurophysiology, 125(9), 1870-1877.

Kontsevich, L. L., \& Tyler, C. W. (1999). Bayesian adaptive estimation of psychometric slope and threshold. Vision Research, 39(16), 2729-2737.

Legrain, V., \& Mouraux, A. (2013). Activating selectively and reliably nociceptive afferents with concentric electrode stimulation: Yes we can! Provided that low stimulus intensities are used! Clinical Neurophysiology, 124(2), 424.

Malmberg, A. B., Mizisin, A. P., Calcutt, N. A., Von Stein, T., Robbins, W. R., \& Bley, K. R. (2004). Reduced heat sensitivity and epidermal nerve fiber immunostaining following single applications of a high-concentration capsaicin patch. Pain, 111(3), 360-367.

Mendell, L. M. (2011). Computational functions of neurons and circuits signaling injury: Relationship to pain behavior. Proceedings of the National Academy of Sciences of the United States of America, 108(SUPPL. 3), 15596-15601.

Moscatelli, A., Mezzetti, M., \& Lacquaniti, F. (2012). Modeling psychophysical data at the population-level: The generalized linear mixed model. Journal of Vision, 12(11).

Mouraux, A., Iannetti, G. D., \& Plaghki, L. (2010). Low intensity intra-epidermal electrical stimulation can activate A $\delta$-nociceptors selectively. Pain, 150(1), 199-207.

Mouraux, A., Marot, E., \& Legrain, V. (2014). Short trains of intra-epidermal electrical stimulation to elicit reliable behavioral and electrophysiological responses to the selective activation of nociceptors in humans. Neuroscience Letters, 561, 69-73.

O'Neill, J., Brock, C., Olesen, A. E., Andresen, T., Nilsson, M., \& Dickenson, A. H. (2012). Unravelling the mystery of capsaicin: A tool to understand and treat pain. Pharmacological Reviews, 64(4), 939-971.

Perkins, F. M., \& Kehlet, H. (2000). Chronic pain as an outcome of surgery. A review of predictive factors. Anesthesiology, 93(4), 1123-1133.

Polydefkis, M., Hauer, P., Sheth, S., Sirdofsky, M., Griffin, J. W., \& McArthur, J. C. (2004). The time course of epidermal nerve fibre regeneration: Studies in normal controls and in people with diabetes, with and without neuropathy. Brain, 127(7), 1606-1615. 
R Core Team. (2014). R: A Language and Environment for Statistical Computing. Vienna, Austria: R Foundation for Statistical Computing. Retrieved from http://www.R-project.org

Ragé, M., Van Acker, N., Facer, P., Shenoy, R., Knaapen, M. W. M., Timmers, M., . . Plaghki, L. (2010). The time course of $\mathrm{CO} 2$ laser-evoked responses and of skin nerve fibre markers after topical capsaicin in human volunteers. Clinical Neurophysiology, 121(8), 1256-1266.

Rollman, G. B. (1969). Electrocutaneous stimulation: Psychometric functions and temporal integration. Perception and Psychophysics, 5(5), 289-293.

Sandkühler, J. (2009). Models and mechanisms of hyperalgesia and allodynia. Physiological Reviews, 89(2), 707-758.

Selim, M. M., Wendelschafer-Crabb, G., Hodges, J. S., Simone, D. A., Foster, S. X. Y. L., Vanhove, G. F., \& Kennedy, W. R. (2010). Variation in quantitative sensory testing and epidermal nerve fiber density in repeated measurements. PAIN ${ }^{\circledast}$ 151(3), 575-581.

Steenbergen, P., Buitenweg, J. R., Trojan, J., van der Heide, E. M., van den Heuvel, T., Flor, H., \& Veltink, P. H. (2012). A system for inducing concurrent tactile and nociceptive sensations at the same site using electrocutaneous stimulation. Behavior Research Methods, 44(4), 924-933.

van der Heide, E. M., Buitenweg, J. R., Marani, E., \& Rutten, W. L. (2009). Single pulse and pulse train modulation of cutaneous electrical stimulation: a comparison of methods. Journal of Clinical Neurophysiology, 26(1), 54-60.

Weiss, G. (1901). Sur la possibilité de rendre comparables entre eux les appareils servant à l'excitation électrique. Turin: H. Loescher.

Woolf, C. J. (2011). Central sensitization: Implications for the diagnosis and treatment of pain. Pain, 152(SUPPL.3), S2-S15.

Zucker, R. S., \& Regehr, W. G. (2002). Short-term synaptic plasticity. Annual Review of Physiology, 64, 355-405. 

Chapter 7

General discussion 
Persistent pain after surgery (PPSP) is a frequently occurring problem. The malfunction of nociceptive mechanisms is thought to be a major contributor to development of PPSP. Increased central sensitization (e.g., hyperalgesia) and decreased descending inhibition (e.g., DNIC) are associated with PPSP. With current observational techniques, such as quantitative sensory testing (QST), it is possible to observe contributions of the nociceptive system to stimulus processing. However, it still remains difficult to distinguish the individual contributions of nociceptive mechanisms, possibly hampering the prediction and treatment of PPSP and other pain disorders. A tool allowing simultaneous observation of contributions of multiple mechanisms to stimulus processing possibly aids in understanding nociceptive mechanisms and, consequently, in the prediction of PPSP.

The primary objective of the work presented in this thesis is to develop a tool allowing the simultaneous observation of individual contributions of nociceptive mechanisms to sensory processing. Two goals are identified in order to achieve the primary objective: 1) develop experimental techniques allowing the simultaneous observation of multiple non-stationary psychophysical thresholds, and (2) characterize the contributions of nociceptive mechanisms to sensory processing.

The use of psychophysical techniques in a threshold tracking paradigm was discussed in Part I of this thesis. In Chapter 2, a comparison between stimulus selection procedures and threshold estimation methods was made by means of computer simulations and a human subject experiment. The experimental finding that nociceptive detection thresholds are non-stationary, even when no conditioning stimulus is applied, is the basis for Chapter 3. There, the effect of a drifting threshold on the estimation quality of psychophysical thresholds and slopes is demonstrated, and a modification to prevent underestimations of the psychophysical slope is presented. In Chapter 4, the tracking paradigm presented in Chapter 2 is extended into a method allowing to simultaneously track multiple thresholds within a single experiment.

The second part of this thesis, Part II, focuses on the application of the experimental methods presented in Part I. The possibility to simultaneously observe contributions of peripheral and central mechanisms to stimulus processing was studied in Chapter 5. In Chapter 6, the responsiveness of thresholds to changes in the nociceptive function was studied. Capsaicin was used to perturb the function of the nociceptive system. 


\section{PSYCHOPHYSICAL METHODS FOR OBSERVATION OF NON-STATIONARY THRESHOLDS}

Existing psychophysical methods consist of applying several stimuli with varying amplitudes, record the corresponding responses (e.g., detected or not detected) and obtain a single estimated threshold. Experimental perturbations, such as a cold pressor test, affect underlying mechanisms and result in dynamic changes in the threshold. A single estimate is, therefore, insufficient to capture these changes. By using a tracking paradigm, similar to the method described by von Dincklage, Hackbarth, Schneider, Baars, and Rehberg (2009), tracking of non-stationairy thresholds could be possible. This possibility was demonstrated in Chapter 2, where nociceptive detection thresholds were tracked before, during, and after a cold pressor test as conditioning stimulus. A temporary increase of about $50 \%$ in the detection threshold was observed within about a minute right after subjects immersed their hand into the water. This increase corresponds to findings in other studies (e.g., Pud, Granovsky, \& Yarnitsky, 2009), and therefore implies that the tracking methodology can be used to detect changes in nociceptive thresholds induced by a conditioning stimulus.

Besides a change in threshold due to a conditioning stimulus, nociceptive thresholds were shown to drift over time (Chapters 2, 3, 5, and 6). One of the required assumptions for psychophysical experiments is that responses to stimuli remain stationary throughout the duration of the experiment (Treutwein, 1995). However, the observed drift implies the assumption of stationarity does not hold. A drift in the threshold decreases the estimation quality of psychophysical parameters (Chapter 3). A modification in the tracking paradigm diminishing this loss in estimation quality was proposed (Chapter 3). Threshold drifts were observed ranging between 0.01 and $0.05[(\mathrm{~mA}) / \mathrm{min}]$. Given an initial threshold of about $0.2 \mathrm{~mA}$ at the start of a 10-minute experiment (Chapter 2), thresholds may have an increase of up to about $150 \%$. The between-subject variability was found to be non-negligible indicating the importance of accounting for drifts. Not accounting for drifting thresholds could potentially affect the observation of within-subject effects. Although not reported in this thesis, within-subject variability of threshold drifts is present as well, resulting in different drifts on different measurement occasions. Again, not accounting for this variability potentially masks the observation of within-subject effects, hampering tailored observation of potential nociceptive disease. Hence, in both group and single-subject analysis, it is recommended to account for possible drifting effects. 
Currently, QST is thought of as a useful tool for clinical applications. For example, the current state of the nociceptive system can be probed by the estimation of pain thresholds and pain tolerance thresholds. While these thresholds could be used to observe the symptoms of nociceptive disease, such as hyperalgesia, it remains difficult to ascribe disease to malfunctioning of a particular nociceptive mechanism. The application of stimuli with various temporal properties could allow more specific probing of underlying processes. Similar to the method presented by Gracely, Lota, Walter, and Dubner (1988), it is possible to present stimuli with various temporal properties within a single experiment and to estimate a threshold corresponding to a set of temporal properties. Combining this method with the tracking paradigm presented in Chapter 2 allows the simultaneous observation of multiple thresholds. As a result, the total available stimuli within an experiment are now divided over the number of tracked thresholds, limiting the observability of temporary changes in nociceptive function. The main limiting factor in the experimental paradigm described in this thesis is the inter-stimulus interval (ISI). The ISI increases when more thresholds are tracked and when subjects need more time to respond to stimuli. Combined with the results presented in Chapters 2, 3, and 4, it is possible to track up to 4 thresholds in a 10-minute experiment where no conditioning stimulus is applied. In experiments including a conditioning stimulus, 2 threshold tracks is a reasonable trade-off between the estimation precision and capability of tracking non-stationaries caused by the cold pressor test (Chapter 4) for the aims in this thesis.

\section{RESPONSIVENESS OF PSYCHOPHYSICAL PARAMETERS TO TEMPORAL STIMULUS PROPERTIES}

Before the methods described in the first part of this thesis can be used to study malfunctions in nociceptive malfunctions, it needs to be demonstrated that these methods can be used to observe individual contributions of nociceptive processes in psychophysical curves. By varying the temporal properties of stimuli (i.e., pulse-width (PW), number of pulses (NoP), and inter-pulse interval (IPI)), it is possible to observe the responsiveness of psychophysical parameters to stimulus properties (Chapter 5). By varying the $\mathrm{PW}$, it was possible to obtain estimates of the strength-duration curve, described by the chronaxie and rheobase. However, as estimates of the strength-duration curve were approximated by the results of only two thresholds, the variability of chronaxie and rheobase estimates between experiments was relatively high. When increasing the NoP from a single pulse to a double pulse stimulus, facilitatory effects were observed resulting in a decrease in the detection probability. Nonetheless, it was unclear at this point whether this facilitation was caused by peripheral mechanisms, central mechanisms, or a combination of both. Only a slight threshold difference was 
observed for IPI values ranging between 10 and $100 \mathrm{~ms}$ (Chapter 5) indicating a small effect of IPI, preventing the observation of possible relevant differences. Future studies could characterize peripheral contributions in double pulse stimuli by, for example, presenting complex stimulus patterns (e.g., Burke et al., 2009). Once characterized, stimulus properties can be chosen such as to minimize the contributions of peripheral processes.

When observing the facilitatory effects in double pulse stimuli, the notion of probability summation should be considered (Chapters 5 and 6). The detection probability of a double pulse stimulus depends on the detection probability of a single pulse, and of possible facilitating and inhibiting processes. In order to determine whether facilitation or inhibition contributes to stimulus processing, an estimate of the threshold and slope of the psychophysical curve is required. Without an estimate of the slope of a single pulse, it is not possible to determine the detection probability for a double pulse stimulus based on pure probability summation. As a result, in order to determine whether facilitation or inhibition is present in a double pulse stimulus, the slope should be estimated as well.

\section{RESPONSIVENESS OF PSYCHOPHYSICAL THRESHOLDS TO CHANGES IN NOCICEPTIVE FUNCTION}

The possibility of observing contributions of nociceptive processing was demonstrated in Chapter 5. The next step was to study the responsiveness of psychophysical thresholds to changes in nociceptive function. The topical application of capsaicin resulted in temporary changes in nociceptive function. The retraction of nociceptive fibres was reflected in increases in thresholds to single pulse stimuli. Even though not discussed in Chapter 6, it can be seen from Figure 6-3 that the difference between the thresholds for different PWs (i.e., setting 1 and setting 2) increased on the second and seventh day in contrast to the control day. This suggests a change over time in the strength-duration relationship, which is expected due to the retraction of nerve fibres. Combining this observation with the observation of a temporary increase in facilitation suggests that the experimental paradigm is capable of observing (pharmacologically-) induced changes in the nociceptive function.

Modulatory effects during the activation of descending mechanisms, for example due to a conditioning stimulus such as the cold pressor test (CPT), were observed in this thesis (Chapter 2 and Chapter 3), but not studied in detail. Activation of descending modulation is thought to have a diffuse inhibiting effect on nociceptive mechanisms 
(Nir \& Yarnitsky, 2015). Consequently, this might lead to the conclusion that CPT induced changes in nociceptive function may not show differences in the threshold estimates with various temporal properties. An effect of the CPT is observed in both a single pulse stimulus and a double pulse stimulus: thresholds were temporarily increased (Chapter 4). Nonetheless, the cold pressor seemed to have a larger effect on single pulse stimuli than on double pulse stimuli, effectively increasing the difference between a single pulse thresholds and double pulse threshold. A possible explanation of this increased difference is a temporary increase in facilitation of the second pulse in a double pulse stimulus. Another, more likely, explanation is a temporary decrease in the slope of the detection probability of a single pulse stimulus. This results in an effective smaller shift of the psychophysical curve at the 0.29 detection probability point than at the 0.5 point (see Chapter 4 for a comprehensive explanation about probability summation). Then, probability summations leads to a smaller effective increase in the threshold for double pulse stimuli as compared to the change in threshold of single pulse stimuli. Estimates of the psychophysical slope could be used to determine whether the CPT has a similar effect on the processing of single pulse stimuli as on double pulse stimuli. This again stresses the relevance of estimating the psychophysical slope.

\section{METHODOLOGICAL REMARKS}

In all the work presented in this thesis, it was assumed that the detection probability of a stimulus could be described by a logistic function. This function was described by two parameters; the threshold and the slope. The biggest advantage of a logistic function is the ease with which it is linearized allowing statistical inferences regarding the position and steepness of the function. A limitation of this choice is revealed when studying probability summation. The expected detection probability of a double pulse stimulus depends on pure probability summation, and thereby on the detection probability of a single pulse stimulus. Moreover, while a logistic function is symmetrical around the threshold point, there are indications that the true underlying function is not symmetrical. The detection probability of a stimulus with an amplitude of $0 \mathrm{~mA}$ is expected to be near zero. However, the estimates presented in Chapter 6 may suggest a higher detection probability at the $0 \mathrm{~mA}$ point (Figure 6-1). One reason for this observation could be the sparseness of available data resulting in poor slope estimates. Another possibility is that the logistic function is a poor choice in describing the underlying detection probability. The use of computational models could provide more insight into the form of the underlying detection probability (e.g., Yang, Meijer, Doll, Buitenweg, \& van Gils, 2015). 
The human subject data presented in the first part of this thesis (Chapter 2 and Chapter 4) were analyzed in two steps. The first step included the tracking paradigm using a shifting/moving window over the available SRPs and applying generalized linear models (logit link function) to obtain momentary estimates of the threshold. Second, the obtained threshold tracks were used in a linear mixed model (LMM) for statistical inferences. While threshold tracks are informative for observation of dynamic properties of underlying processes, it requires an additional step before statistical inferences can be made. Moreover, due to the tracking paradigm, information regarding the slope of the psychophysical function is lost. Therefore, a generalized linear mixed model with a logit link function can be used for statistical inferences using the SRPs, instead of using the processed data (i.e., threshold tracks). This allows, for example, the estimation of the slope parameter. On the other hand, interpretation of the regression coefficients is complicated when using GLMMs, as an additional transformation (i.e., inverse logit) is required before psychophysical curves can be obtained.

\section{CLINICAL APPLICABILITY}

The statistical models to observe the effects of study parameters presented in this thesis, whether these were threshold tracks or SRPs, were within-subject models and estimated both between and within-subject variances. The primary aim of these models was to observe possible effects of study parameters. Individual assessment of subjects' nociceptive function is therefore, at this point, difficult. The combination of a psychophysical experiment with a computational model describing relevant neurophysiological components could improve the individual assessment of nociceptive function. One of the requirements for such a setup is the choice of optimal electrical stimulus parameters. As the available time for obtaining stimulus response pairs is limited, only up to 2 or 4 different sets of stimulus parameters can be chosen. The choice of stimulus parameters is therefore limited, and should be adjusted according to the study population. For example, when studying patients with peripheral disease (e.g., small fiber neuropathy), observing contributions of peripheral processes might be most informative. Another example is patients scheduled for total knee arthroplasty (TKA), where the focus is more on central ascending and descending contributions to stimulus processing.

\section{GENERAL CONCLUSION}

The research presented in this thesis provides psychophysical methods for improved observation of nociceptive processing. With the developed methods, it is possible to simultaneously observe the contributions of various nociceptive processes. Based on the results described in this thesis, several recommendations are provided for setting 
up and performing relatively short psychophysical experiments. An experiment lasting for about 30 minutes can contribute to observation of peripheral and central processes, as well as the effect of a conditioning stimulus on these processes. The duration can be shortened to about 10 minutes when the effect of a conditioning stimulus is irrelevant. Future studies should focus on further validation of the methods by (1) studying the responsiveness of psychophysical curves to other pharmacological interventions in healthy human subjects, (2) combining it with a computational model based on underlying mechanisms, and (3) studying the predictability of post-surgical chronic pain. Doing so could demonstrate additional value of the presented methods for clinical applications.

\section{REFERENCES}

Burke, D., Howells, J., Trevillion, L., McNulty, P. A., Jankelowitz, S. K., \& Kiernan, M. C. (2009). Threshold behaviour of human axons explored using subthreshold perturbations to membrane potential. Journal of Physiology, 587(Pt 2), 491-504.

Gracely, R. H., Lota, L., Walter, D. J., \& Dubner, R. (1988). A multiple random staircase method of psychophysical pain assessment. Pain, 32(1), 55-63.

Nir, R. R., \& Yarnitsky, D. (2015). Conditioned pain modulation. Current Opinion in Supportive and Palliative Care, 9(2), 131-137.

Pud, D., Granovsky, Y., \& Yarnitsky, D. (2009). The methodology of experimentally induced diffuse noxious inhibitory control (DNIC)-like effect in humans. Pain, 144(1-2), 16-19.

Treutwein, B. (1995). Adaptive psychophysical procedures. Vision Research, 35(17), 2503-2522.

von Dincklage, F., Hackbarth, M., Schneider, M., Baars, J. H., \& Rehberg, B. (2009). Introduction of a continual RIII reflex threshold tracking algorithm. Brain Research, 1260, 24-29.

Yang, H., Meijer, H. G. E., Doll, R. J., Buitenweg, J. R., \& van Gils, S. A. (2015). Computational modeling of Adeltafiber-mediated nociceptive detection of electrocutaneous stimulation. Biological Cybernetics, 109(4-5), 479-491. 



\section{SUMMARY}

The perception of pain plays a crucial role in our daily life. Pain as a result of (potential) tissue damage, also called nociception, warns and protects the body from (further) damage. Normally, acute pain, as a result of tissue damage, disappears along with the healing process. In some cases, however, persisting pain outlasts the healing process and no longer serves as a protecting mechanism. When the pain lasts longer than 3 to 6 months, the pain is considered as chronic and negatively affects the quality of life.

The prevalence rate of chronic pain worldwide is estimated to range between $10 \%$ and $55 \%$ and is estimated to be about $18 \%$ in the Netherlands. A common cause for chronic pain is surgery. The estimated prevalence rates depend on the type of surgical intervention and range between $30 \%$ and $50 \%$ in major surgical interventions and range between $10 \%$ and $15 \%$ in minor surgical interventions. Pain treatments are, once chronic pain has established, relatively ineffective: less than a third of patients achieve a $50 \%$ pain reduction. A growing body of literature suggests a link between nociceptive malfunction and chronic post-surgical pain.

The nociceptive system can be roughly divided into ascending pathways from peripheral nociceptors through the spinal cord into the brain, and descending pathways from the brain through the spinal cord to the dorsal horn. The sensitizing mechanisms in ascending pathways respond to nociceptive stimuli within tens of milliseconds, whereas inhibiting mechanisms in descending pathways respond slower, varying between a minute and several hours. In healthy humans, the sensitizing and inhibiting mechanisms are balanced resulting in a return to baseline functionality after termination of nociceptive input. However, an imbalance, either by increased sensitization or by decreased inhibition, results in enhanced nociception, possibly leading to chronic pain.

For the observation of nociceptive stimulus processing and sensory function, psychophysical quantitative sensory testing (QST) methods are used. These tests are used for the estimation of sensory thresholds using a broad range of stimulus types, such as thermal, mechanical, or electrical. The estimated thresholds can be used to identify nociceptive disease. However, it remains difficult to obtain information about the individual contributions of underlying mechanisms to stimulus processing. Furthermore, a single thresholds estimate cannot be used to observe changes in the threshold as a result of changes in the nociceptive system. By presenting stimuli with various temporal stimulus properties (e.g., pulse-width, number of pulses, or inter-pulse interval) and 
estimating corresponding thresholds, the individual contributions of peripheral and central nociceptive processes to stimulus processing might be distinguished. Moreover, tracking these thresholds over time might help observing changes in the nociceptive system. Combined, this could contribute to an improved observation of nociceptive processing.

The primary objective of the work presented in this thesis is to develop a tool allowing the simultaneous observation of individual contributions of nociceptive mechanisms to sensory processing. Two goals are identified in order to achieve the primary objective: 1) develop experimental techniques allowing the simultaneous observation of multiple non-stationary psychophysical thresholds, and (2) characterize the contributions of nociceptive mechanisms to sensory processing. This thesis is subdivided into two parts, each part addressing one of the goals.

\section{PART I}

\section{Psychophysical methods for observation of sensory function}

The first part of this thesis focuses on the development of experimental techniques and addresses three methodological issues.

Chapter 2 discusses how a psychophysical threshold can be efficiently tracked over time. With the aid of computer simulations and a human subject experiment, the performance of several stimulus selection procedures and threshold estimation methods were compared. During the human subject experiment, subjects were to temporarily immerse their hand into cold water. This painful stimulus (i.e., conditioning stimulus) caused temporary changes in the nociceptive system. As a result of the conditioning stimulus, a temporarily increased threshold was observed. Moreover, a continuous drift in thresholds was observed. This implied that within an experiment, thresholds cannot be assumed to be stationary.

The effect of a drifting threshold on the estimation quality of the psychophysical threshold and slope is presented in Chapter 3. Computer simulations of a psychophysical experiment were used to assess this effect. While estimates of the threshold were relatively unaffected by threshold drifts, the estimates of the slope were severely underestimated. A modification to the tracking paradigm discussed in Chapter $\mathbf{2}$ was presented to improve the estimation quality of the psychophysical curve. Finally, the stimulus response pairs coming from two human subjects were used to demonstrate this improvement. 
Chapter 4 introduces the concept of simultaneously tracking multiple non-stationary thresholds within a single experiment. The use of this method in an experiment including healthy human subjects demonstrated the possible usefulness. However, the results of this experiment also suggested that tracking multiple thresholds affects 1) the speed with which changes in thresholds can be observed, and (2) the estimation quality. The limitations of this method were studied by means of computer simulations of psychophysical experiments. The results were incorporated into recommendations for the implementation of experiment designs where multiple thresholds are simultaneously tracked.

\section{PART II}

Observation of nociceptive processing using electrocutaneous stimulation

In Part II, the methods described in Part I are applied to study the contributions of peripheral and central mechanisms to nociceptive sensory processing. Part II consists of two chapters.

In Chapter 5, it is studied whether the contributions of nociceptive processes can be observed in psychophysical curves. The responses to electrical stimuli with various temporal properties were recorded in a human subject experiment. The results demonstrate that psychophysical curves can be used to observe contributions of peripheral and central processes. At this point, however, the contributions of peripheral and central processes cannot be distinguished with certainty.

As a first step towards a clinical application, the responsiveness of thresholds to changes in the nociceptive function was studied in Chapter 6. A human subject experiment was performed where the function of the nociceptive system was perturbed by capsaicin (8\%). The effect of the perturbation lasted for a period of about 3 months. Nociceptive detection thresholds were estimated for stimuli with various temporal properties prior to and on several occasions after the perturbation. The detection thresholds for single-pulse stimuli returned to baseline within 28 days (an increase was observed on days 2 and 7), whereas the threshold for double-pulse stimuli were increased on days 7 and 28 . This suggests that changes in peripheral and central processes can be observed with the methods described in this thesis.

\section{GENERAL CONCLUSIONS}

The research presented in this thesis provides psychophysical methods for improved observation of nociceptive processing. With the developed methods, it is possible to 
simultaneously observe the contributions of various nociceptive processes. Based on the results described in this thesis, several recommendations are provided for setting up and performing relatively short psychophysical experiments. An experiment lasting for about 30 minutes can contribute to observation of peripheral and central processes, as well as the effect of a conditioning stimulus on these processes. The duration can be shortened to about 10 minutes when the effect of a conditioning stimulus is irrelevant. Future studies should focus on further validation of the methods by (1) studying the responsiveness of psychophysical curves to other pharmacological interventions in healthy human subjects, (2) combining it with a computational model based on underlying mechanisms, and (3) studying the predictability of post-surgical chronic pain. Doing so could demonstrate additional value of the presented methods for clinical applications. 



\section{SAMENVATTING}

Pijn speelt een cruciale rol in ons bestaan. Pijn als gevolg van (dreigende) weefselschade, ofwel nociceptieve pijn, waarschuwt de mens om erger te voorkomen. Acute pijn als gevolg van weefselschade verdwijnt normaal gesproken tijdens het helingsproces. Aanhoudende pijn, ook wel persisterende pijn genoemd, heeft de waarschuwende functie verloren. De kwaliteit van leven wordt door deze pijn negatief beïnvloedt, zeker als de pijn langer dan 3 tot 6 maanden aanhoudt (chronische pijn).

Het aantal mensen dat wereldwijd aan chronische pijn lijdt wordt tussen de 10\% en $55 \%$ geschat, in Nederland is dit naar schatting circa $18 \%$. Een veelvoorkomende oorzaak van chronische pijn is een chirurgische ingreep. De prevalentie van postoperatieve chronische pijn hangt af van het type ingreep. De schattingen van het aantal mensen met chronische pijn na een operatie variëren tussen de 30\% en 50\% bij zware ingrepen, en tussen de $10 \%$ en $15 \%$ bij lichte ingrepen. Pijnbestrijding blijkt lastig, minder dan een derde van de patiënten met chronische pijn ervaart na behandeling een pijnverlichting van $50 \%$ of minder. Steeds vaker wordt in de literatuur een relatie gelegd tussen het slecht functioneren van het nociceptieve systeem en chronische postoperatieve pijn.

Het nociceptieve systeem kan ruwweg worden verdeeld in opgaande paden van perifere nociceptoren, die via de dorsale hoorn in het ruggenmerg verbonden zijn met de hersenen, en neergaande paden van de hersenen door het ruggenmerg richting de dorsale hoorn. Waar sensitiserende mechanismen in opgaande paden binnen tientallen milliseconden kunnen reageren op nociceptieve prikkels, reageren de inhiberende mechanismen in neergaande paden trager, variërend tussen een minuut en uren. Bij gezonde mensen zijn de mechanismen in de opgaande en de neergaande paden in balans. Ze zorgen er mede voor dat de pijn verdwijnt zodra de nociceptieve input stopt. Een onbalans, als gevolg van verhoogde sensitisatie of verlaagde inhibitie, kan leiden tot versterkte nociceptie, wat vervolgens kan leiden tot chronische pijn.

Om inzicht te krijgen in nociceptieve stimulusverwerking en het sensorisch functioneren worden psychofysische kwantitatieve sensorische testen (QST) gebruikt. Met deze testen worden de sensorische drempels geschat voor verschillende typen stimuli zoals thermische, mechanische of elektrische. Aan de hand van deze sensorische drempels is het mogelijk om nociceptieve ziekten te identificeren. Doordat deze testen een enkele drempel bepalen, is het moeilijk binnen een experiment informatie in te winnen over de individuele bijdragen van verschillende onderliggende nociceptieve mechanismen 
aan de stimulusverwerking. Een enkele drempelschatting is daarnaast onvoldoende om veranderingen van de drempel, als gevolg van veranderingen binnen het nociceptieve systeem, te observeren. Door binnen een experiment de temporele eigenschappen van stimuli (bijvoorbeeld puls-breedte, aantal pulsen en interpuls-interval) te variëren en bijbehorende drempels te schatten kunnen mogelijk de individuele bijdragen van perifere en centrale processen aan de stimulusverwerking geobserveerd worden. Door de drempels in de tijd te volgen kunnen ook veranderingen in het nociceptieve systeem geobserveerd worden. Tezamen kan dit bijdragen aan een betere observeerbaarheid van nociceptieve ziekten.

De primaire doelstelling van het onderzoek van dit proefschrift is het ontwikkelen van een instrument dat het mogelijk maakt simultaan verschillende bijdragen van het nociceptieve systeem aan de verwerking van stimuli te observeren. Er zijn twee subdoelen gesteld: (1) het ontwikkelen van experimentele technieken voor het simultaan observeren van meerdere niet-stationaire psychofysische drempels, en (2) het karakteriseren van bijdragen van nociceptieve mechanismen aan de verwerking van stimuli. Deze doelen worden in twee afzonderlijke delen van dit proefschrift behandeld.

\section{DEEL I}

\section{Psychophysical methods for observation of sensory function}

In het eerste deel van dit proefschrift wordt ingegaan op het ontwikkelen van experimentele technieken en worden drie methodologische vraagstukken behandeld.

Centraal in Hoofdstuk 2 staat het vraagstuk hoe een psychofysische drempel zo efficiënt mogelijk in de tijd gevolgd kan worden. Daartoe zijn, met behulp van computersimulaties en een experiment met gezonde proefpersonen, de prestaties van diverse stimulusselectieprocedures en drempelschattingsmethoden vergeleken. Tijdens dit experiment moesten de proefpersonen tijdelijk een hand in ijswater houden. Deze pijnlijke stimulus, ook wel conditionerende stimulus genoemd, zorgde voor tijdelijke veranderingen in het nociceptieve systeem. Als gevolg van de conditionerende stimulus trad een tijdelijke verhoging van de drempel op. Daarnaast werd een geleidelijk verschuivende drempel geobserveerd. Daarmee werd duidelijk dat stationariteit van een drempel binnen een experiment niet kan worden verondersteld.

Het effect van een geleidelijk verschuivende drempel op de schattingskwaliteit van de drempel en de steilheid van de psychofysische curve is het onderwerp van Hoofdstuk 3. Dat effect is beoordeeld aan de hand van simulatiemodellen met verschuivende 
drempels. Hoewel de schattingen van de drempels relatief onaangetast bleven, was de geschatte steilheid van de psychofysische curve aanzienlijk lager dan die van het model. Voor een betere schatting van de psychofysische curve wordt een aanpassing van de in Hoofdstuk 2 beschreven methode voorgesteld. Ten slotte zijn stimulus-respons paren van twee proefpersonen gebruikt om deze verbetering te demonstreren.

In Hoofdstuk 4 wordt een methode geïntroduceerd voor het simultaan volgen van meerdere niet-stationaire drempels. Bij het gebruik van deze methode in een experiment met gezonde proefpersonen is gebleken dat het volgen van meerdere drempels gevolgen heeft voor (1) de snelheid waarmee veranderingen in de drempel geobserveerd kunnen worden, en (2) de schattingskwaliteit van drempels. De beperkingen van deze methode zijn verder onderzocht met behulp van computermodellen van psychofysische experimenten. De resultaten zijn verwerkt in aanbevelingen voor het opzetten van experimenten waarin meerdere drempels simultaan gevolgd kunnen worden.

\section{DEEL II}

\section{Observation of nociceptive processing using electrocutaneous stimulation}

In het tweede deel van dit proefschrift worden de methoden, die beschreven zijn in Deel I, toegepast om de bijdragen van perifere en centrale mechanismen aan nociceptieve sensorische verwerkingen te onderzoeken.

In Hoofdstuk 5 wordt onderzocht of de bijdragen van nociceptieve processen kunnen worden geobserveerd in psychofysische curves. In een experiment zijn de responsies van gezonde proefpersonen op elektrische stimuli met verschillende temporele eigenschappen gemeten. De resultaten laten zien dat de psychofysische curves gebruikt kunnen worden om bijdragen van perifere en centrale processen te observeren. De bijdragen van perifere en centrale processen zijn op dit moment echter nog niet met zekerheid te onderscheiden.

Als eerste stap richting de klinische toepasbaarheid van de ontwikkelde methode is de responsiviteit van nociceptieve drempels op veranderingen in de nociceptieve functie onderzocht. Dit wordt beschreven in Hoofdstuk 6. In een experiment met gezonde proefpersonen is de functie van het nociceptieve systeem door middel van capsaïcine (8\%) verstoord voor een periode van ongeveer 3 maanden. Voorafgaand, en op verschillende momenten na het veroorzaken van deze verstoring, zijn de nociceptieve detectiedrempels geschat voor stimuli met variërende temporele eigenschappen. Het onderzoek liet zien dat de detectiedrempel voor stimuli bestaande uit een enkele puls 
binnen 28 dagen terugkeerde naar baseline waarde (op dagen 2 en 7 was een verhoging geobserveerd), terwijl drempels voor stimuli bestaande uit twee pulsen op dagen 7 en 28 verhoogd waren. Dit suggereert dat ook veranderingen in perifere en centrale processen met de ontwikkelde methode kunnen worden geobserveerd.

\section{ALGEMENE CONCLUSIES}

Vanuit de behoefte aan meer inzicht in de (dynamische) processen van het nociceptieve systeem zijn in dit onderzoek psychofysische methoden ontwikkeld. Met de ontwikkelde methoden is het mogelijk geworden om bijdragen van verschillende nociceptieve processen simultaan te observeren. Vanuit het onderzoek volgen diverse aanbevelingen voor het opzetten en uitvoeren van een psychofysisch experiment. In een relatief kort experiment van circa 30 minuten kunnen bijdragen van perifere en centrale processen geobserveerd worden, evenals het effect van een conditionerende stimulus op die processen. Wanneer een conditionerende stimulus niet relevant is voor het uit te voeren onderzoek kan de experimentduur verkort worden tot ongeveer 10 minuten. Het is aan te bevelen dat toekomstige studies zich richten op een nadere validatie van de methoden door (1) het bestuderen van de responsiviteit van psychofysische curves tijdens andere farmacologische ingrepen in gezonde proefpersonen, (2) het combineren van de methoden met een wiskundig model dat de onderliggende mechanismen beschrijft, en (3) het bestuderen van de voorspelbaarheid van postoperatieve chronische pijn. Uit deze onderzoeken zal blijken wat de mogelijke meerwaarde is van de besproken methoden voor klinische toepassingen. 



\section{DANKWOORD}

Met de afronding van mijn proefschrift in zicht, is het tijd voor dit laatste woord. Ik wil graag iedereen bedanken die, op wat voor manier dan ook, bijgedragen heeft aan mijn proefschrift. Een aantal personen wil ik in het bijzonder bedanken.

Allereerst wil ik mijn dagelijks begeleider en copromotor Jan Buitenweg bedanken. Ondanks mijn onzekerheid over mijn plek binnen de universiteit, toonde jij al snel vertrouwen in mij, door mij kort na mijn individuele opdracht te wijzen op deze promotieplek. Dankzij dit vertrouwen en de vrijheid die je mij gaf, kon ik een eigen draai geven aan het onderzoek. En hoewel er diverse hobbels te overwinnen waren, denk ik dat er mooie resultaten behaald zijn die ruimte bieden voor vervolg. Dank voor de mogelijkheden, de discussies en je feedback.

Natuurlijk wil ik ook mijn promotor, Peter Veltink, bedanken. Ik heb onze discussies erg gewaardeerd. Jouw feedback op manuscripten, die je vaak al op korte termijn kon geven, was zeker wat betreft het aanwijzen van zwakke plekken zeer nuttig.

Mijn promotieonderzoek maakte deel uit van het PaINSIGHT project, een samenwerking tussen de Universiteit Twente (BSS en AAMP), CHDR, Radboudumc en het St. Antonius ziekenhuis. Van de AAMP-groep wil ik vooral Hil Meijer en Huan Yang bedanken. Hil, ik heb onze samenwerking, zeker rondom mijn eerste artikel en de discussies over stimulusparameters, als prettig en nuttig ervaren. Huan, your enthusiasm and perseverance for understanding every little detail has been a motivation to me during the last few years. I enjoyed the time we spent explaining and discussing the relevance of our work to each other. Vanuit het CHDR wil ik graag Geert Jan Groeneveld, Justin Hay, Guido van Amerongen en Pieter Okkerse bedanken voor hun wetenschappelijke bijdragen en de door hen geboden ruimte om aan te haken bij twee studies. Het meewerken en het overdragen van 'mijn' opstelling was voor mij zeer leerzaam. Kris Vissers en Oliver Wilder-Smith (Radboudumc) wil ik bedanken voor de nuttige bijdragen tijdens de vergaderingen van de gebruikerscommissie. Lajla Kadic en Tijn Bouw wil ik bedanken voor hun inspanningen om een studie binnen het Radboudumc van de grond te krijgen. Eric van Dongen en Imre Krabbenbos (St. Antonius ziekenhuis): dank voor de mogelijkheid om een studie bij jullie in het St. Antonius ziekenhuis uit te voeren. Ook was het prettig om voor een paar maanden met jullie te mogen werken. 
Binnen de vakgroep Biomedische Signalen en Systemen wil ik iedereen bedanken voor de koffiepauzes, lunchwandelingen en $3^{\text {e }}$ - en $5^{\text {e }}$-donderdag van de maand' borrels. Wies, bedankt voor alle administratieve hulp en gezellige gesprekken. Sandra, bedankt voor jouw administratieve hulp in de laatste periode van mijn aanstelling. Ed en Marcel, jullie wil ik graag bedanken voor alle technische ondersteuning. Het heeft mij erg geholpen dat jullie altijd klaar stonden wanneer er bijvoorbeeld weer eens iets midden in een studie gerepareerd moest worden, of wanneer er binnen een paar dagen tientallen elektroden gebouwd moesten worden. Wat betreft technische ondersteuning wil ik Victor niet vergeten. De niet bijzonder fotogenieke 'perturbator' is toch nog zeer nuttig gebleken. Ook wil ik jou en Frodo bedanken voor de hulp bij alle perikelen van de stimulatoren. Binnen de vakgroep heb ik redelijk wat kamergenoten gehad. In het bijzonder wil ik graag Vishi, Peter en Gerco noemen. Vishi, thanks for all your freeof-charge advice and discussions. Peter, dankzij jou ben ik geïnteresseerd geraakt in de statistiek. Ik vond het prettig om met je te discussiëren over methoden en het opzetten van modellen. Gerco, ik heb genoten van onze gesprekken omtrent de politieke en economische misstanden in deze wereld.

Dit proefschrift had hier nooit gelegen zonder alle mensen die hebben deelgenomen aan de studies. Het merendeel heeft behalve het aanvoelen van talloze elektrische prikkels ook - vrijwillig - een hand voor een aantal minuten in pijnlijk ijswater gestoken. Dank voor deze bereidwilligheid! Dit brengt mij ook gelijk bij de studenten die ik afgelopen jaren heb mogen begeleiden. Floor, Sjoerd, Bart en Marc: bedankt voor jullie bijdragen aan het onderzoek. Ik vond de samenwerking met jullie erg leuk en leerzaam. Naast experimenten met menselijke proefpersonen heb ik ook simulaties van experimenten uitgevoerd. Daarvoor wil ik iedereen bedanken die dit simulatieproces versneld heeft door mij een aantal processor cores ter beschikking te stellen.

Graag wil ik ook al mijn vrienden bedanken voor de mij gegeven benodigde afleiding. Een aantal vrienden wil ik apart noemen. Arjan (paranimf), ondanks de behoorlijke hoeveelheid water tussen onze huizen hebben we ons contact toch goed boven water weten te houden. Bedankt voor de interessante gesprekken over wetenschap, muziek en nooit-aan-begonnen-projectjes. Joris (paranimf) en Fokke, bedankt voor de gezellige en serieuze gesprekken en de etentjes. Het tripje naar Legoland was geweldig. Djurre, Joyce en Mark: bedankt voor de grote hoeveelheden pannenkoeken en de slechte films. Bas en Rhinus: bedankt voor de weekenden met goede stukken vlees. Ik geloof dat we de vakantie naar Portugal en Spanje (grotendeels betaald door TAP Portugal) voorlopig niet zullen vergeten. Sanne, bedankt voor de koppen thee, de koeken en je luisterend 
oor. Lamia, thanks for the dinners and nice conversations we had. Roel, bedankt voor het eten en de uurtjes gamen. Lara, bedankt voor alle steun. Dan wil ik verder nog kort noemen: Marjolein, Rob, en Melanie; bedankt.

Lisa, hoewel je op een druk moment binnen bent komen lopen, hoop ik niet dat ik jouw beeld van promoveren beïnvloed heb. Je hebt het voor mij de afgelopen tijd veel leuker gemaakt. Als laatste wil ik mijn familie bedanken. Zonder de gezelligheid en steun over de jaren heen zou ik nooit zo ver gekomen zijn. Opa en Oma, jullie zijn altijd een inspiratie voor mij geweest. Pap en Mam, bedankt voor de onvoorwaardelijke vrijheid, steun en mogelijkheden die jullie mij gegeven hebben. 



\section{BIOGRAPHY}

Robert-Jan Doll was born on July 26, 1988 in 's-Gravenhage, the Netherlands. He finished his HAVO education at Van der Capellen scholengemeenschap (Zwolle) in 2005. He studied electrical engineering at the University of Applied Sciences Windesheim (2005-2009) in Zwolle. He did an internship at BMA Ergonomics (Zwolle) and an internship at Thales (Hengelo). After his graduation project in 2009, also at Thales, on the design of board schematics and signal integrity analysis of high speed signals, he continued his studies in electrical engineering at the University of Twente (Enschede): first a pre-master (2009-2010) followed by a master in electrical engineering (2010-2012). During his masters, he started working on his PhD thesis at the Biomedical Signals and Systems group (November 2010), also at the University of Twente. He received his Master of Science degree in 2012 after finishing his master thesis entitled: 'Comparison of adaptive psychophysical methods for nociceptive threshold tracking: a simulation and human subject study'.

Robert-Jan's PhD research focused on developing psychophysical methods for observation of nociceptive processing using electrical intra-epidermal stimulation. His research was part of the PaINSIGHT projectand was a collaboration between the University of Twente, Centre for Human Drug Research (Leiden), Radboudumc (Nijmegen), and the St. Antonius hospital (Nieuwegein). 


\section{LIST OF PUBLICATIONS}

\section{JOURNAL PAPERS}

Doll, R.J., Buitenweg, J.R., Meijer, H.G.E., Veltink, P.H. (2014). Tracking of nociceptive thresholds using adaptive psychophysical methods. Behavior Research Methods, 46(1), 55-66.

Doll, R.J., Veltink, P.H., Buitenweg, J.R. (2015). Observation of time-dependent psychophysical functions and accounting for threshold drifts. Attention, Perception, \& Psychophysics, 77(4), 1440-1447.

Doll, R.J., Maten, A.C.A., Spaan, S.P.G., Veltink, P.H., Buitenweg, J.R. (2016). Effect of temporal stimulus properties on the nociceptive detection probability using intraepidermal electrical stimulation. Experimental Brain Research, 234(1), 219-227.

Yang, H., Meijer, H.G.E., Doll, R.J., Buitenweg, J.R., van Gils, S.A. (2015). Biological Cybernetics, 109(4), 479-491.

Doll, R.J., Veltink, P.H., Buitenweg, J.R. Characterization of a psychophysical method for simultaneous tracking of multiple non-stationary thresholds. Submitted.

Doll, R.J., van Amerongen, G., Hay, J.L., Groeneveld, G.J., Veltink, P.H., Buitenweg, J.R. Responsiveness of electrical nociceptive detection thresholds to capsaicin (8\%) induced changes in nociceptive processing. Submitted.

Yang, H., Meijer, H.G.E., Doll, R.J., Buitenweg, J.R., van Gils, S.A. Dependence of nociceptive detection thresholds on physiological parameters and capsaicin-induced neuroplasticity: a computational study. Submitted.

\section{CONFERENCE CONTRIBUTIONS}

Doll, R.J., Maten, A.C.A., Spaan, S.P.G., Veltink, P.H., Buitenweg, J.R. (2015). Nociceptive detection probability depends on the temporal properties of electrocutaneous stimuli. Fifth Dutch Bio-Medical Engineering Conference, Egmond aan Zee, the Netherlands.

Doll, R.J., Veltink, P.H., Buitenweg, J.R. (2015). Differentiating nociceptive mechanisms using electrocutaneous detection thresholds. Fifth Dutch Bio-Medical Engineering Conference 2015, Egmond aan Zee, the Netherlands.

Doll, R.J. (2014). Observation of multiple nociceptive thresholds: the effect of temporal stimulus properties and capsaicin. NeuroSIPE symposium, Soesterberg, the Netherlands. 
Doll, R.J., Meijer, H.G.E., Buitenweg, J.R. (2013). Multiple threshold tracking methods for improved observation of nociceptive function. Fechner Day 2013, Freiburg i.Br., Germany.

Doll, R.J., Buitenweg, J.R., van Amerongen, G., Hay, J.L., Groeneveld, G.J., Veltink, P.H. (2013). Capsaicin (8\%) patch increases multiple electrical nociceptive thresholds in healthy human subjects. The 8th EFIC Pain Congress, Florence, Italy.

Doll, R.J. (2013). Observation of nociceptive thresholds in human subjects during a painful stimulus. NeuroSIPE symposium, Doorn, the Netherlands.

Doll, R.J., Buitenweg, J.R., Meijer, H.G.E., Veltink, P.H. (2013). Simultaneous tracking of multiple nociceptive thresholds: a simulation and human subject study. Fourth Dutch Bio-Medical Engineering Conference, Egmond aan Zee, the Netherlands.

Doll, R.J., Buitenweg, J.R., Veltink, P.H. (2012). Comparison of adaptive psychophysical methods for the use of nociceptive threshold tracking: a simulation and human subject study. Neuroscience 2012, New Orleans, LA, USA.

Doll, R.J., Buitenweg, J.R., Veltink, P.H. (2012). Comparison of adaptive psychophysical methods for the use of nociceptive threshold tracking: a simulation and human subject study. NeuroSIPE summer-school, Chicago, IL, USA.

Doll, R.J. (2012). Development of methods for dynamical threshold tracking. NeuroSIPE symposium, Soesterberg, the Netherlands.

Doll, R.J., Yang, H., Meijer, H.G.E., Buitenweg, J.R. (2011). Simulation of psychophysical stimulus selection procedures for dynamic threshold tracking. Tenth Belgian Day on Biomedical Engineering, Brussels, Belgium.

Yang, H., Doll, R.J., Meijer, H.G.E., Buitenweg, J.R. (2011). Modeling and identifying the ascending sensory system with the psychophysical binary responses and electrocutaneous stimuli. Tenth Belgian Day on Biomedical Engineering, Brussels, Belgium.

Buitenweg, J.R., Doll, R.J. (2011). Threshold sensitivity to diffuse noxious inhibitory controls depends on electrocutaneous stimulus properties. The 14th European Congress on Clinical Neurophysiology, Rome, Italy. 
